University of Tennessee Health Science Center

UTHSC Digital Commons

\title{
Sex-related differences in perception and discrimination of different speakers: An analysis of the auditory dorsal stream via EEG
}

David Thornton

University of Tennessee Health Science Center

Follow this and additional works at: https://dc.uthsc.edu/dissertations

Part of the Speech Pathology and Audiology Commons

\section{Recommended Citation}

Thornton, David (0000-0002-1562-986X), "Sex-related differences in perception and discrimination of different speakers: An analysis of the auditory dorsal stream via EEG" (2019). Theses and Dissertations (ETD). Paper 486. http://dx.doi.org/10.21007/etd.cghs.2019.0478.

This Dissertation is brought to you for free and open access by the College of Graduate Health Sciences at UTHSC Digital Commons. It has been accepted for inclusion in Theses and Dissertations (ETD) by an authorized administrator of UTHSC Digital Commons. For more information, please contact jwelch30@uthsc.edu. 


\title{
Sex-related differences in perception and discrimination of different speakers: An analysis of the auditory dorsal stream via EEG
}

\author{
Abstract \\ Growing evidence that speech perception tasks elicit sensorimotor activity, and that this activity varies \\ due to context, sex, cognitive load, and cognitive ability. However, it is unknown as to whether the sex of \\ the speaker and demands of the task differentially effect males and females during speech perception \\ tasks. This study investigated whether speaker sex and task demands (i.e. passive listening or active \\ discrimination) influence sensorimotor and auditory cortical activity in males and females differently. Raw \\ EEG data were collected from 27 males and 29 females during passive listening to, and discrimination of \\ $/ \mathrm{ba} / \mathrm{and} / \mathrm{da} /$ syllable pairs spoken by a synthetic female or male speaker. Independent component \\ analysis identified sensorimotor and auditory components characterized by alpha/beta and alpha peaks, \\ respectively. Time- frequency decomposition revealed no significant differences between male and \\ female groups in any testing conditions. Within the male group, stronger mu activity was found in active \\ discrimination conditions compared to passive listening in only the left hemisphere, while females \\ displayed stronger activity in both the left and right hemisphere. Auditory cluster activity revealed males \\ utilizing stronger inhibition after stimulus offset in active discrimination conditions, while females showed \\ stronger activation during stimulus presentation in the same conditions. Both males and females \\ displayed stronger mu activation in response to male speakers compared to female speakers before, \\ during, and after stimulus presentation. Given this, it appears that speaker sex does influence at least \\ anterior dorsal stream activity in a similar fashion for both males and females, but task demands \\ differentially alter anterior and posterior dorsal stream activity in each sex group. These findings may at \\ least partially explain the high variability in findings across neuroimaging studies that feature males and \\ females in the same population.

\section{Document Type} \\ Dissertation \\ Degree Name \\ Doctor of Philosophy (PhD) \\ Program \\ Speech and Hearing Science \\ Research Advisor \\ Ashley W. Harkrider, Ph.D. \\ Keywords \\ Cognition, Electroencephalography, Sex Differences, Speech Perception, Time-frequency Analysis \\ Subject Categories \\ Communication Sciences and Disorders | Medicine and Health Sciences | Speech Pathology and \\ Audiology
}


Sex-related Differences in Perception and Discrimination of Different Speakers: An Analysis of the Auditory Dorsal Stream via EEG

\author{
A Dissertation \\ Presented for \\ The Graduate Studies Council \\ The University of Tennessee \\ Health Science Center
}

In Partial Fulfillment

Of the Requirements for the Degree

Doctor of Philosophy

From The University of Tennessee

By

David Thornton

May 2019 
Copyright (C) 2019 by David Thornton. All rights reserved. 


\section{DEDICATION}

For my wife, my family, my friends, and my pets. Without such tireless support behind me, I would not have made this happen. 


\section{ACKNOWLEDGEMENTS}

This work would not have been completed without the support of many family, friends, colleagues, and mentors. This is by no means an exhaustive list, but I will do my best to acknowledge and extend my gratitude as many people as I can.

To my mentor, Dr. Ashley W. Harkrider. From the beginning, I could talk to Dr. Harkrider about any idea I had for a research project. To her credit, she never told me how uninformed I was, or how many times over these ideas had been approached. Her encouragement to dig deeper, read more, and work harder to find new questions pushed me to this point. I would not be here without her tireless guidance, support, encouragement, and feedback.

To my committee members, Drs. Saltuklaroglu, Johnstone, Plyler, and Buss, for their advice and guidance on my projects. To Dr. Saltuklaroglu particularly for putting up with my challenges to ideas and always listening, especially when I developed a knack for finding all the things that weren't working.

To my wife, Kristen Thornton, for listening to me when I needed to vent, for her constant support, and for offering constructive feedback on writing and project ideas. Without her, I would have struggled with continued motivation and determination. She provided, and continues to provide, a steady hand and a calming influence that pushes me to work harder than I thought I could.

To my parents and my sister, Paul and Wanda Thornton, and Susan Oyola, for cheering me on from afar. For pushing me to not give up when the going got tough, and for helping to keep my head on straight.

To my longtime friend Nicholas Vercruysse, for providing a laugh every now and again, and late nights of video games when stress was at all-time high. Such escapes allowed for greater focus when work resumed.

To my lab colleague, Dave Jenson, for the many cathartic complaint sessions. I believe that neither of us would have finished without the other there to keep us in check and moving forward.

Finally, to Blake Rafferty, for coming to me with questions and trusting my answers. But more importantly for coming in to the lab and always being ready to help with anything and everything that needed to get done. The ongoing projects are in good hands. 


\begin{abstract}
Growing evidence that speech perception tasks elicit sensorimotor activity, and that this activity varies due to context, sex, cognitive load, and cognitive ability. However, it is unknown as to whether the sex of the speaker and demands of the task differentially effect males and females during speech perception tasks. This study investigated whether speaker sex and task demands (i.e. passive listening or active discrimination) influence sensorimotor and auditory cortical activity in males and females differently. Raw EEG data were collected from 27 males and 29 females during passive listening to, and discrimination of /ba/ and /da/ syllable pairs spoken by a synthetic female or male speaker. Independent component analysis identified sensorimotor and auditory components characterized by alpha/beta and alpha peaks, respectively. Timefrequency decomposition revealed no significant differences between male and female groups in any testing conditions. Within the male group, stronger mu activity was found in active discrimination conditions compared to passive listening in only the left hemisphere, while females displayed stronger activity in both the left and right hemisphere. Auditory cluster activity revealed males utilizing stronger inhibition after stimulus offset in active discrimination conditions, while females showed stronger activation during stimulus presentation in the same conditions. Both males and females displayed stronger mu activation in response to male speakers compared to female speakers before, during, and after stimulus presentation. Given this, it appears that speaker sex does influence at least anterior dorsal stream activity in a similar fashion for both males and females, but task demands differentially alter anterior and posterior dorsal stream activity in each sex group. These findings may at least partially explain the high variability in findings across neuroimaging studies that feature males and females in the same population.
\end{abstract}




\section{TABLE OF CONTENTS}

CHAPTER 1. INTRODUCTION ................................................................................

CHAPTER 2. LITERATURE REVIEW .....................................................................6

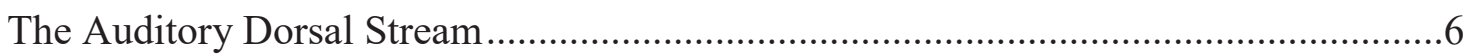

fMRI Investigation of the Anterior Auditory Dorsal Stream .....................................

Uneven Participant Distribution and Listener/Speaker Differences ............................ 10

Electroencephalography (EEG) and the Importance of Temporal Sensitivity ..............13

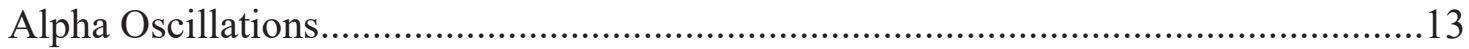

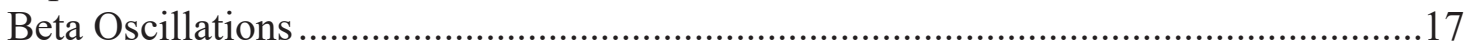

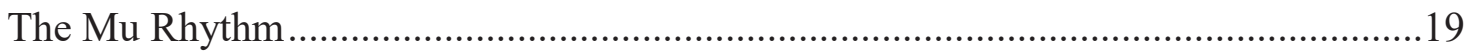

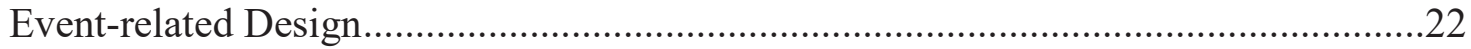

Auditory-to-Motor Mapping and Sensorimotor Processing ....................................22

Measurement of Sensorimotor and Cognitive Processes via EEG and Sex-related

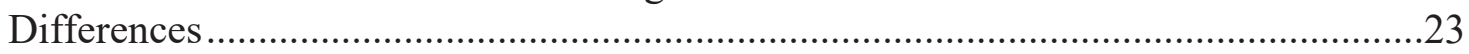

Sensorimotor Activity in Speech Perception ...........................................................26

The Auditory Cortex and Sex-related Differences ..............................................29

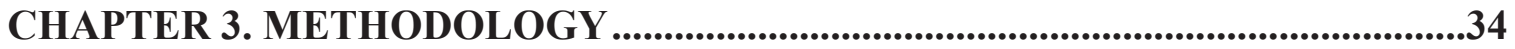

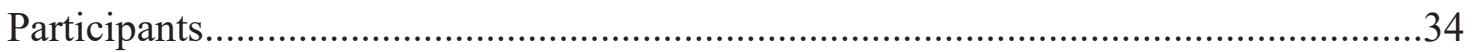

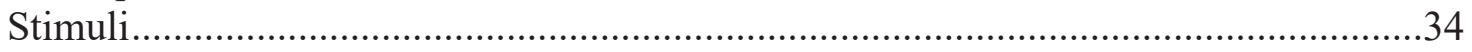

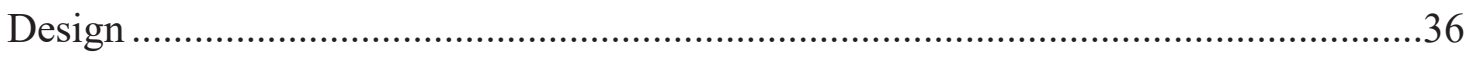

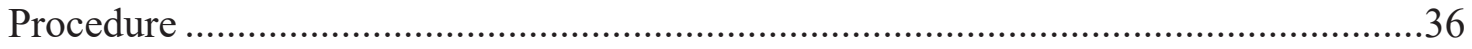

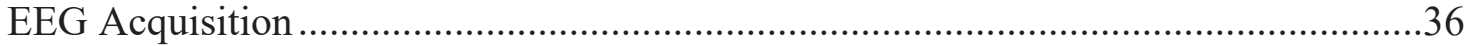

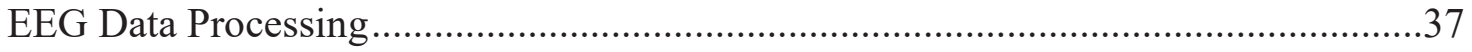

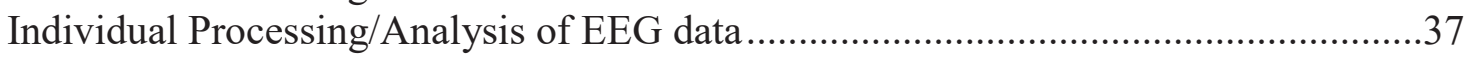

Independent Component Analysis (ICA) of EEG Data ..........................................38

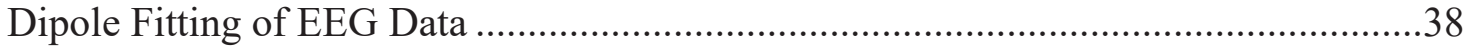

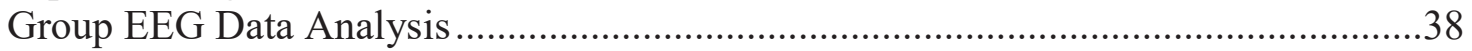

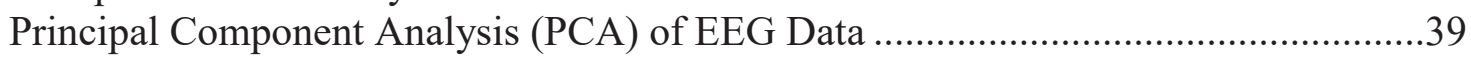

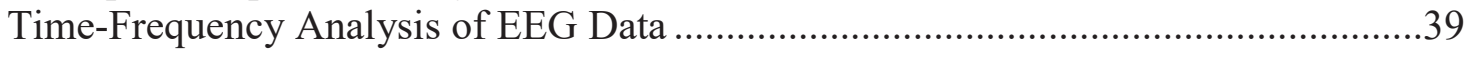

CHAPTER 4. RESULTS...............................................................................................40

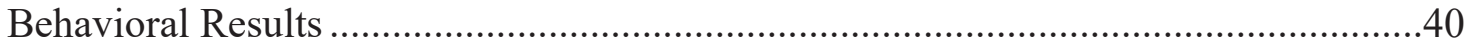

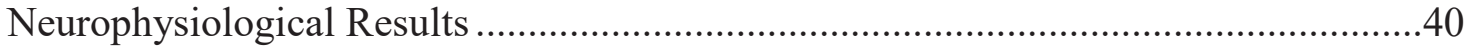

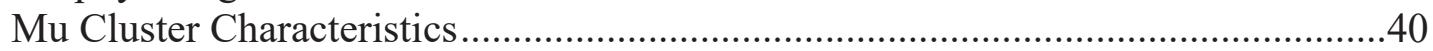

Auditory Cluster Characteristics ............................................................................. 40

Does Speaker Sex Influence Anterior Dorsal Stream Processing? ............................42

Does Increasing Task Demands Increase Dorsal Stream Activity?..........................42

The Effects of Speaker Sex Within Group ............................................................50 


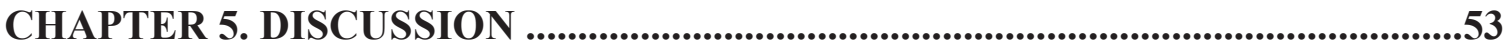

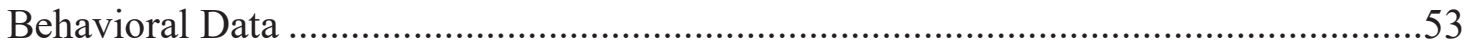

The Influence of Speaker Sex on Anterior Dorsal Stream Processing …………...........53

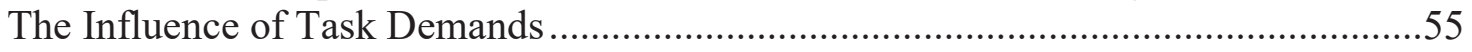

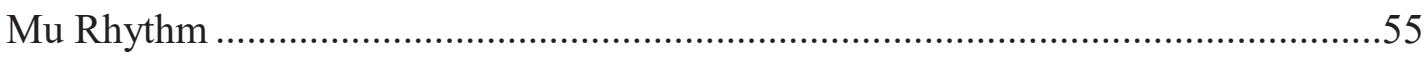

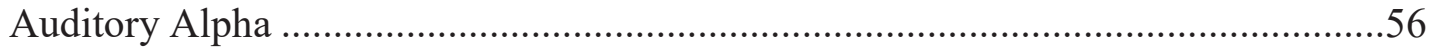

Differential Effects of Speaker Sex Revealed Within Each Group ..............................56

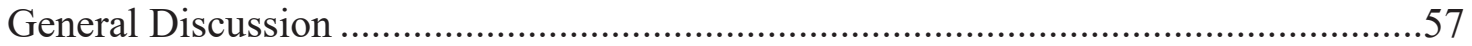

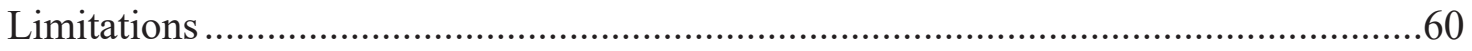

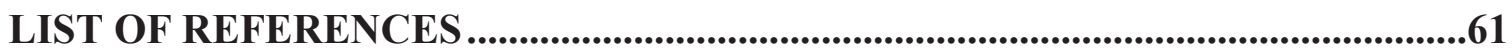

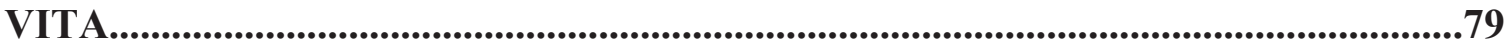




\section{LIST OF TABLES}

Table 4-1. Source Localization Information for Mu Clusters. ....................................41

Table 4-2. Source Localization of Auditory Clusters...............................................43 


\section{LIST OF FIGURES}

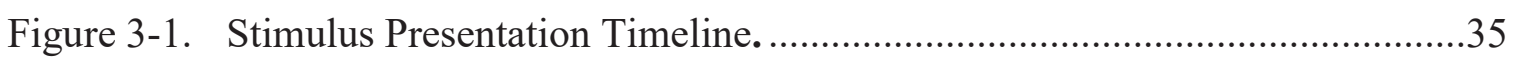

Figure 4-1. Left Hemisphere Mu Cluster Time-Frequency Analysis.............................44

Figure 4-2. Right Hemisphere Mu Cluster Time-Frequency Analysis..........................45

Figure 4-3. Left Hemisphere Mu Cluster Time-Frequency Analysis Task

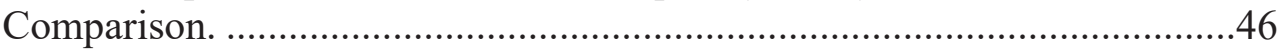

Figure 4-4. Right Hemisphere Mu Cluster Time-Frequency Analysis Task

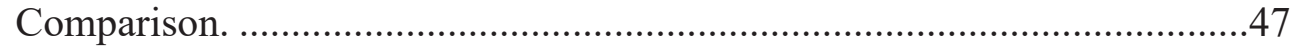

Figure 4-5. Left Hemisphere Auditory Cluster Time-Frequency Analysis Task

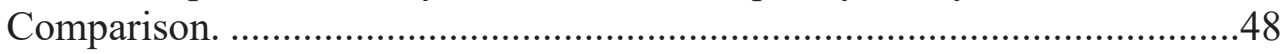

Figure 4-6. Right Hemisphere Auditory Cluster Time-Frequency Analysis Task Comparison.

Figure 4-7. Left Hemisphere Mu Cluster Time-Frequency Analysis Speaker Comparison.

Figure 4-8. Right Hemisphere Mu Cluster Time-Frequency Analysis Speaker Comparison. 


\section{LIST OF ABBREVIATIONS}

$\begin{array}{ll}\text { ABR } & \text { Auditory brainstem response } \\ \text { ANOVA } & \text { Analysis of variance } \\ \text { AWS } & \text { Adults who stutter } \\ \text { AxS } & \text { Analysis-by-synthesis } \\ \text { CON } & \text { Passive listening to white noise } \\ \text { CV } & \text { Consonant-vowel } \\ \text { DAN } & \text { Dorsal attention network } \\ \text { DF } & \text { Discrimination of female speaker } \\ \text { DM } & \text { Discrimination of male speaker } \\ \text { EEG } & \text { Electroencephalography } \\ \text { ERD } & \text { Event-related desynchronization } \\ \text { ERP } & \text { Event-related potential } \\ \text { ERSP } & \text { Event-related spectral perturbations } \\ \text { ERS } & \text { Event-related synchronization } \\ \text { FDR } & \text { False discovery rate } \\ \text { fMRI } & \text { Functional magnetic resonance imaging } \\ \text { GBI } & \text { Gating-by-inhibition } \\ \text { ICA } & \text { Independent component analysis } \\ \text { MEG } & \text { Magnetoencephalography } \\ \text { MMN } & \text { Mismatch negativity } \\ \text { PCA } & \text { Principal component analysis } \\ \text { PET } & \text { Positron emission tomography } \\ \text { PF } & \text { Passive listening to female speaker } \\ \text { PM } & \text { Passive listening to male speaker } \\ \text { RMS } & \text { Root-mean-square } \\ \text { RT } & \text { Response time } \\ \text { SNR } & \text { Signal-to-noise ration } \\ \text { TFS } & \text { Typically fluent speakers } \\ \text { TMS } & \text { Transcranial magnetic stimulation } \\ \text { WMN } & \text { Working memory network } \\ & \end{array}$




\section{CHAPTER 1. INTRODUCTION}

The auditory dorsal stream consists of the auditory cortices, premotor cortex, and inferior parietal lobe. Speech perception tasks routinely elicit activation of the anterior portion of the auditory dorsal stream, the premotor/primary motor cortical areas (Bartoli et al., 2015; Bowers, Saltuklaroglu, Harkrider, \& Cuellar, 2013; D’Ausilio, Bufalari, Salmas, \& Fadiga, 2012; Pulvermuller et al., 2006; Saltuklaroglu, Harkrider, Thornton, Jenson, \& Kittilstved, 2017; Thornton, Harkrider, Jenson, \& Saltuklaroglu, 2017). This region is thought to be responsible for sensorimotor processing tasks associated with speech, such as the mapping of auditory input on the articulatory gestures with which the listener would recreate the signal (Fowler, Brown, Sabadini, \& Weihing, 2003; Galantucci, Fowler, \& Turvey, 2006). Neuroimaging studies which reveal activation within this region utilize tasks varying in cognitive demands. Speech perception studies often inherently recruit processes such as categorical discrimination (Callan, Callan, Gamez, Sato, \& Kawato, 2010; Jenson et al., 2014), working memory (M W Burton, Small, \& Blumstein, 2000; Martha W. Burton \& Small, 2006; LoCasto, Krebs-Noble, Gullapalli, \& Burton, 2004), and attention (Bowers et al., 2013; Callan, Callan, \& Jones, 2014; Jenson et al., 2014; Popovich, Dockstader, Cheyne, \& Tannock, 2010) in order to complete tasks. Given the anatomical overlap of several networks with the anterior auditory dorsal stream with portions of the dorsal attention network (DAN; Vossel, Geng, \& Fink, 2014) and the working memory network (WMN; Baddeley, 2012; Koshino, Minamoto, Yaoi, Osaka, \& Osaka, 2014), activation of these regions could be due to any of these processes. This led researchers to ask whether the activation seen by these studies is due to cognitive demands, or if activity is present when passively listening. Should activity be present when cognitive demands are minimal or absent, it would suggest that there is some automatic recruitment of the dorsal stream which support models of speech perception that suggest the decoding of articulatory gestures to perceive speech.

Passive listening/viewing studies have utilized both visual and auditory speech signals to investigate 'automatic' activation of the anterior dorsal stream to reduce or eliminate the confounding effects of cognitive demands. Mottonen and colleagues (2005) report enhanced responses using magnetoencephalography (MEG) across the somatosensory cortex when participants view speech signals. Listening to speech, however, did not modulate these responses. Several functional magnetic resonance imaging (fMRI) studies have revealed 'automatic' activation of the anterior dorsal stream to speech based stimuli using audition only (Pulvermüller et al., 2006; Skipper, Nusbaum, \& Small, 2005; Skipper, Van Wassenhove, Nusbaum, \& Small, 2007; Wilson, Saygin, Sereno, \& Iacoboni, 2004). Differences in activation of the anterior dorsal stream to speech presented via audition is found when listening to non-native language phonemes compared to native language phonemes (Wilson \& Iacoboni, 2006). Activation of the anterior dorsal stream during passive speech perception was also found using EEG by Oliveira and colleagues (2017). Taken together, these studies suggest that passive viewing and/or listening to speech signals is sufficient to recruit the anterior dorsal stream, possible for direct mapping of sensory stimuli onto articulatory gestures. 
Conversely, several studies do not reveal activation of the anterior auditory dorsal stream when passively listening. A review from Scott and colleagues (2009) suggested that when the whole brain is scanned during a speech perception task, little or no motor activity is revealed. Several fMRI studies from Belin and colleagues (Belin, Zatorre, \& Ahad, 2002; Belin, Zatorre, Lafaille, Ahad, \& Pike, 2000) support this claim, and found no anterior dorsal stream activity in passive listening to speech signals, which have been supported by findings from Bowers and colleagues (2013), using electroencephalography (EEG). Indeed, lesions to the anterior dorsal stream regions appear to have little to no effect on speech perception outcomes (Hickok, Costanzo, \& Capasso, 2011). Conflicting findings suggest that in addition to task, there may be other sources of variability in the anterior dorsal stream activity during passive and/or active speech perception, possibly the sex of the speaker and/or the sex of the listener.

Many studies, regardless of passive or active tasks, have more participants who are male or who are female (Bowers et al., 2013; Jenson et al., 2014; Möttönen, Dutton, \& Watkins, 2013; Skipper et al., 2005; Thornton et al., 2017; Wilson \& Iacoboni, 2006), or have so few participants that statistical comparisons would be unlikely to reveal sex differences (Ojanen et al., 2005; Pulvermüller et al., 2006; Skipper et al., 2005). Few studies have considered the extent to which differences in processing related to perception may exist and be related to the sex of either the listener or speaker. Bartoli and colleagues (2015) examined listener/speaker effects using transcranial magnetic stimulation (TMS) across the anterior dorsal stream while participants listened to and identified CV syllables. The authors suggested that listeners actively compare incoming speech signals with their own motor production templates. Facilitation of $\mathrm{CV}$ identification (response time; RT) was stronger when the perceived "perceptual distance" between listener and speaker was smaller. Simply put, male listeners performed with faster RTs when listening to male speakers due to the reduced "perceptual distance", and vice versa.

Regarding speaker differences, many studies utilize a female speaker for the stimuli and examine resultant activity in both males and females (Möttönen et al., 2005; Ojanen et al., 2005; Pekkola et al., 2006; Pulvermüller et al., 2006; Skipper et al., 2007) while others employ male speaker stimuli (Jenson, Harkrider, Thornton, Bowers, \& Saltuklaroglu, 2015a; Thornton et al., 2017; Wilson \& Iacoboni, 2006; Wilson et al., 2004). Many of these studies have a maximum of 10-15 participants split between each sex, reducing the likelihood of accurate between group comparisons. To this end, Gootjes and colleagues (2006) utilized a female speaker in a study examining regions of the auditory dorsal stream and revealed patterns of inhibitory activity in males using MEG. In contrast, Thornton and colleagues' (2018) utilized a male speaker and revealed inhibitory activity in females. As such, questions remain about whether recruitments of the anterior dorsal stream is influenced by the sex of the listener, the sex of the speaker, the task, or some combination thereof. To investigate the influence of these sources of variability, it is necessary to examine the effects of tasks (passive listening to or discrimination of speech), speaker sex, and listener sex. fMRI studies have provided the cortical region to investigate, however given the dynamic recruitment of the anterior dorsal stream, examining regional activity with greater temporal sensitivity is necessary. 
EEG possesses strong temporal resolution, enabling investigation of cortical activity preceding, during, and after presentation of a stimulus. This allows for interpretation of cognitive activity related to the stimulus processing and task. Easily measurable with EEG is the sensorimotor mu rhythm, which is characterized by peaks of activity in the alpha $(8-13 \mathrm{~Hz})$ and beta $(15-25 \mathrm{~Hz})$ frequency bands (Hari, 2006; Schnitzler, Gross, \& Timmermann, 2000). These bands are associated with cognitive (alpha and beta) (Chen \& Huang, 2016; Klimesch, 2012; Lundqvist et al., 2015), sensory (alpha) (Foxe \& Snyder, 2011; Quandt, Marshall, Bouquet, \& Shipley, 2013; Weisz, Hartmann, Müller, Lorenz, \& Obleser, 2011), and motor (beta) (Arnal, 2012; Quandt, Marshall, Shipley, Beilock, \& Goldin-Meadow, 2012; Tzagarakis, Ince, Leuthold, \& Pellizzer, 2010) processes. Broadly, when power increases from baseline measures in a frequency band, it is termed event-related synchronization (ERS), and thought to reflect cortical inhibition. Decreases in power, event-related desynchronization (ERD), represents release from inhibition for the purpose of active processing (Klimesch, 2012; Pfurtscheller \& Lopes, 1999). The mu rhythm is primarily localized to the anterior dorsal stream region, making it ideal for disambiguating cognitive and 'automatic' sensorimotor processes. Any power fluctuations within the mu rhythm occurring before stimulus onset should reflect early attention based activity, while activity following stimulus offset would likely be linked to working memory processes. Activity during stimulus presentation would be indicative of continued, ongoing attention, or sensorimotor processes.

Jenson et al. (2014) investigated mu rhythm activity before, during, and after discrimination of speech sounds. Accurate discrimination of the stimuli revealed mu-beta ERD before, during, and after stimulus onset. Alpha activity just before and during stimulus presentation was characterized by ERS, which gave way to ERD after stimulus offset. Early, concurrent alpha ERS and beta ERD was suggested to represent a motor based model was being used to predict and evaluate incoming sensory information against a prediction. This prediction "narrows" sensory analysis, thus ERS is present in the alpha band. Post stimulus ERD in both alpha and beta was thought to represent working memory to retain task relevant information. Saltuklaroglu et al. (2017) examined this activity in a group of adults who stutter (AWS) and a control group. Using the same stimuli and tasks, this study revealed similar post-stimulus offset activity, with both alpha and beta characterized by ERD. Interestingly, the activity just before and during stimulus presentation was not as strongly characterized by alpha ERS, even beta ERD appeared to be weaker than that found in Jenson et al. (2014). Of note, 17 of 20 participants in Jenson et al. (2014) were female, while 19 of 27 participants in Saltuklaroglu et al. (2017) were male. This led investigators to consider the possibility that sex-related differences may exist within these conditions.

To investigate this further, Thornton et al., (in prep) examined mu rhythm activity during active discrimination of synthetic male speech stimuli. Behavioral performance was high in both males and females, as such, any mu rhythm differences would not be due to behavioral outcomes, and could indicate different processing strategies used to complete the same task. Specifically, males are characterized by negligible alpha ERS 
activity just before and during stimulus presentation when speech is presented in quiet, while females displayed strong alpha ERS both in quiet and in noisy background conditions. Males, when listening to speech in quiet, were characterized by prestimulus beta ERD which extended into stimulus presentation while females did not reveal such activity. No differences between males and females were noted following stimulus offset. The results suggest that males and females utilize early attention allocated to the stimuli differently in the anterior dorsal stream in ideal listening conditions, while the introduction of noise eliminates sex-related differences. This study did not test the interaction between the sex of the listener and the sex of the speaker, a possibility considering results from Gootjes et al. (2006).

Evidence from Thornton et al. (in prep) revealed sex differences in sensorimotor processing during speech discrimination. The presence of sex-related differences in the activity from anterior dorsal stream regions raises the question of possible sex-related differences in activity from other regions of the dorsal stream. An initial study, using positron emission tomography (PET) has revealed that auditory cortical activity is different in males and females even when processing white noise (Ruytjens et al., 2007). Activity in the auditory cortex is of particular interest as it serves as the initial node from which information is first evaluated. Several models (Skipper, Devlin, \& Lametti, 2017; Skipper et al., 2007) of dorsal stream communication suggest interaction between anterior and posterior regions communicate throughout the analysis of auditory signals. Oscillatory activity in the auditory cortex is commonly characterized by suppression of the alpha rhythm, indicating active processing of auditory stimuli (Weisz \& Obleser, 2014). Jenson et al., (2015) revealed alpha activation when listening to speech stimuli, followed by alpha inhibition suggesting reduction of activity in the auditory cortex when no stimulus was presented. The timing of these fluctuations in the auditory alpha rhythm were examined in the context of the timing of activity within the anterior dorsal stream. As alpha ERS disappeared in the anterior dorsal stream, alpha ERD arose in the auditory region. While no direct measures of connectivity were used, this was thought to indicate intercommunication between regions, particularly considering that ERD after stimulus offset in the anterior dorsal stream coincided with ERS in the auditory region.

The overall goal of this study is to characterize sex-related differences in neural activity from several nodes (anterior: premotor, posterior: auditory) of the auditory dorsal stream during passive listening to and active discrimination of speech signals in ideal listening conditions (i.e. silence). To do this, sensorimotor mu rhythm and auditory alpha activity to investigate automatic auditory-to-motor processes will be recorded from males and females via EEG during active discrimination tasks and passive listening using male and female speakers. Active discrimination is associated with attention and working memory, while passive listening is associated with a reduction or removal of cognitive demands. Generally, it is expected that speaker sex will interact with listener sex to influence patterns of neural activity over the time course of the speech stimulus, during both passive listening and active discrimination conditions. More specifically, alpha ERS is expected before and during speech presentation in conditions where participants listen to or discriminate speech produced by a speaker of the opposite sex, while ERD is expected when the speaker is the same sex as the listener before and during stimulus 
presentation. Findings from this study will expand the understanding of auditory dorsal stream dynamics that may be differentiated by sex while informing current theories of speech perception and the role of the anterior dorsal stream (i.e. constructivism, direct realism). The results of this study may discourage generalization of neuroimaging studies. Clinically, sex-related differences investigated here may explain, at least in part, why certain neurodevelopmental disorders characterized by inhibitory deficits are more often diagnosed in males. 


\section{CHAPTER 2. LITERATURE REVIEW}

\section{The Auditory Dorsal Stream}

The dual stream model of auditory speech processing posits the presence of two cooperative streams, ventral and dorsal, tasked with different portions of speech analysis (Hickok \& Poeppel, 2000, 2004, 2007. The ventral stream originates in the auditory cortex and extends forward to the inferior frontal gyrus and is associated with mapping sound to meaning. The auditory dorsal steam is thought to be composed of the posterior auditory regions, inferior parietal lobe, and anterior premotor/primary motor regions of the cortex (Hickok \& Poeppel, 2000, 2004, 2007). This stream is linked to locating sound sources in space as well as assisting with perception of speech when the listening situation isn't ideal (i.e. noise degraded; premotor/primary motor cortex) (Du, Buchsbaum, Grady, \& Alain, 2014; Du, Buchsbaum, Grady, \& Alain, 2016; Murakami, Kell, Restle, Ugawa, \& Ziemann, 2015).

Activation of the anterior dorsal stream (premotor/primary motor cortex) in degraded listening conditions has given rise to several theories. Harkening back to the motor theory of speech perception (Liberman \& Mattingly, 1985), the anterior dorsal stream was thought to map acoustic information to articulatory gestures for the purpose of overcoming the 'lack of invariance' problem. This is the result of such sources of variability as speaker identity (e.g. male, female, child) (Hillenbrand, Getty, Clark, \& Wheeler, 1995), context (sounds occurring around the phoneme influencing production) (Lisker \& Abramson, 1967), and differing speech conditions (speed of speech) (Nygaard \& Pisoni, 1995).

While the strict interpretation of the role of the anterior dorsal stream in speech perception provided by the motor theory of speech perception is largely considered extreme, it has given rise to other explanations which support varying degrees of utility to this region for speech perception. Analysis-by-synthesis (AxS; Poeppel \& Monahan, 2011; Stevens and Halle, 1967) suggests that previous experience with sensory input is used to generate predictions about the content of upcoming information. Further studies suggested that these predictions are generated by the anterior dorsal stream and sent to the posterior auditory regions where the signal is compared to incoming information (Skipper et al., 2005). When predicted information matches incoming information, no additional cortical processing is required, though if the prediction is not matched, additional corrective analyses must be performed (Skipper et al., 2017, 2007).

Direct realist theories are more akin to traditional motor theory suggesting that speech is mapped on to the articulatory gestures with which the listener would re-create the sounds (Fowler et al., 2003; Galantucci et al., 2006). This idea suggests that sounds are relayed from the auditory cortical region of the dorsal stream toward the premotor cortex for where auditory-to-motor transformation occurs. This activity is thought to support perception by activation of stored gestures to overcome the lack of invariance problem. In this case, the acoustic signals specifies the articulatory gesture in which it 
was formed, and that these gestures are the basic units of speech perception (Fowler et al., 2003).

There are theoretical reasons for dorsal stream activity in speech perception that are not specific to speech perception processing and rely more on general cognitive processes which overlap anatomically with the anterior dorsal stream regions. The premotor/primary motor cortical region is a member of several proposed processing networks, the aforementioned auditory dorsal stream (Hickok \& Poeppel, 2000, 2004, 2007), the dorsal attention network (Vossel et al., 2014), and the working memory network (Baddeley, 2012).

The dorsal attention network extends from the occipital lobe anteriorly through the intraparietal sulcus and terminates at the frontal eye field (FEF: Vossel et al., 2014). In Brodmann's area terms, the FEF is housed in BA 8, which can be considered to be the most anterior portion of the auditory dorsal stream (Rauschecker \& Scott, 2009). Given this, a common explanation for anterior dorsal stream recruitment in speech perception and discrimination tasks is attention toward relevant stimuli. The anterior dorsal attention network is most often investigated in visual task paradigms which reveal consistent activation (Vossel et al., 2014; Zumer, Scheeringa, Schoffelen, Norris, \& Jensen, 2014)), thus, it is likely that similar regional activation could be found in response to other stimulus modalities including audition and somatosensation. To this end, auditory stimulation using both non-speech and speech signals have elicited activation from this region using functional magnetic resonance imaging (fMRI; Burton, 2001; LoCasto et al., 2004) and electroencephalography (EEG; Bowers et al., 2013; Jenson et al., 2014; Thornton et al., 2017). It remains plausible, then, that revealed activity could simply be the result of attention.

Many studies employ a task to keep participants attentive and to later examine accuracy and response times for some brain-behavior link. These tasks inherently require working memory, as participants must retain information and then use it to inform an eventual response. The working memory network consists of regions of the precentral gyrus as well as the superior parietal lobe (Eriksson, Vogel, Lansner, Bergström, \& Nyberg, 2015). This anterior overlap with the auditory dorsal stream once again suggests that activity related to speech processing in the region may simply be related to the task involving some working memory process. Visual (Pessoa, Gutierrez, Bandettini, \& Ungerleider, 2002), auditory (Kumar et al., 2016), and somatosensory (Savini, Brunetti, Babiloni, \& Ferretti, 2012) studies which entail some working memory component have all revealed what could be considered "anterior dorsal stream activation" despite this clear overlap. It appears as though any suggestion that activity found in the anterior auditory dorsal stream is related to hypothesis generation or auditory-to-motor mapping could be easily encompassed or obscured by attention and/or working memory demands.

Disentangling the effects of attention, working memory, and sensorimotor processing occurring in the anterior dorsal stream requires specific implementation of passive tasks. While active tasks necessarily invoke attention and working memory as information must be attended to, retained, and used in a response, passive tasks reduce 
the cognitive demands imposed by both of these processes. Activation of the anterior dorsal stream to passive listening tasks involving speech signals should be the result of some processes largely unrelated to attention or working memory, and thus would be encompassed, perhaps, by hypothesis generation based on previous experience $(\mathrm{AxS})$ or direct auditory-to-motor mapping, the activation of articulatory gestures utilized to recreate perceived stimuli.

\section{fMRI Investigation of the Anterior Auditory Dorsal Stream}

Active discrimination tasks often reveal patterns of activity across the pre- and post-central gyrus, consistent with recruitment of the anterior dorsal stream (Burton, 2001; Burton \& Small, 2006; LoCasto et al., 2004; Murakami et al., 2015; Osnes, Hugdahl, \& Specht, 2011; Rogers, Mottonen, Boyles, \& Watkins, 2014; D’Ausilio, Pulvermuller, et al., 2009; D’Ausilio et al., 2012; Ojanen et al., 2005). Passive tasks, on the other hand, provide more mixed results. Though there are examples which found minimal or no activity in passive tasks (Belin et al., 2002; Belin et al., 2000; Scott et al., 2009), several studies counter this finding with varying degrees of activation to both visual and auditory stimuli passively experienced.

Möttönen and colleagues (2005) had participants view speech, listen to speech, while receiving lower lip stimulation, or median nerve stimulation. Eight participants were recruited for this study, with one being female. For the conditions involving a speaker being viewed or listened to, a female speaker was used. All conditions were passive, with no response required by the participant. This study revealed activation of the anterior dorsal region when viewing speech, as well as when lip stimulation occurred, specifically in the left hemisphere. The results were interpreted as suggestive of "embodied simulation" where viewing of the articulatory movements of a speaker's mouth reflexively activated the regions of the viewer's cortex similarly associated with that production. This simulation is effectively a mapping of viewed articulatory gestures to the articulatory repertoire of the viewer, linking perception of speech to production of speech. While this study did not reveal similar activity when listening to speech, instead suggesting that auditory stimuli were insufficient to recruit speech motor areas, other work has argued that auditory stimuli may recruit anterior dorsal stream activity as well.

The mapping of perceived speech signals on to articulatory gestures relies on a very close relationship between the motor production system and the perception system. Pulvermüller et al., (2006) investigated this idea utilizing an event-related fMRI design involving auditory presentation of $/ \mathrm{p} /$ and $/ \mathrm{t} /$ sounds, and subsequent production of these syllables from the participants in a later scan. Each sound was spoken by a female, and the task was performed by seven females and five males. No explicit response was required, and no explicit tracking of the stimulus was implied, so this task is considered to be passive. The temporal cortex was most strongly activated by the speech perception, but critically, speech motor regions were recruited in regions associated with the manner of production for each sound (Pulvermüller et al., 2006). This study tentatively linked the production system for specific phonemes with the perception system for these phonemes. 
The findings indicate that when listening to speech sounds the corresponding regions of the cortex which are recruited to produce the same phonemes are reflexively recruited. Whether that activity is functional or epiphenomenal is not approached by the authors, and without additional testing using transcranial magnetic stimulation, such investigation would be difficult to perform.

Skipper and colleagues (2005) used fMRI to examine regional brain activation while participants listened to, watched, or watched and listened to a story. It is unclear whether the story teller was male or female based on the detail provided by the authors. Five females and four males completed the tasks. Audiovisual stimulation activated cortical areas including the superior temporal gyrus and sulcus, the pars opercularis, primary motor cortex, premotor cortex, somatosensory cortex, and the cerebellum. Activation of the premotor region was modulated by the amount of distinguishable phonemes in the story. It is important to note that activation of these regions was seen in the visual alone and auditory alone conditions, but this activity was significantly weaker than combined stimulation. The authors suggest that auditory and visual information is sufficient to map on to articulatory-motor gestures, but that the increased information provided by audiovisual stimuli facilitates this process and thus supports greater levels of activity in the anterior dorsal stream regions (Skipper et al., 2005).

Expanding on the findings from Skipper and colleagues (2005), the follow-up study from the same laboratory examined visual only, auditory only, and audiovisual presentation of syllables. Stimuli were created using a female speaker, and consisted of $/ \mathrm{pa} /, / \mathrm{ka} /$, and $/ \mathrm{ta} /$. No active participation was required by the participants. The fMRI experiment was performed by 21 participants, with no mention of the number of males and females. Passive perception of speech activated frontal motor areas most strongly in the audiovisual condition, but the auditory and visual only conditions did reveal similar regional activation patterns. The authors suggest that speech signals elicit a motor plan in the listener which would reproduce the phoneme intended by the speaker. The information from this motor plan is sent to the auditory cortex (referred to as corollary discharge) where it influences the interpretation of the sensory percept (Skipper et al., 2007). This explanation appears to be a combination of some prediction generation akin to AxS, but relies on auditory-to-motor transforms based on the content of a signal to generate motor-based predictions about upcoming sensory information. This occurs in the absence of an explicit task, suggesting that this process is reflexively recruited in the presence of speech signals.

Removing the influence of visual processing, Wilson et al., (2004) recruited ten participants to listen to male speaker produce /gi/ and subsequently produce the syllable in a second scan. The participant pool was four females and six males. Active regions were monitored via fMRI and included the superior portion of the ventral premotor cortex as well as the expected temporal areas during the passive speech perception condition. The ventral premotor portion overlapped with speech production areas including the premotor and primary motor regions of the cortex. The authors indicate that the motor system is involved in speech perception and attribute the regional activity as auditory-to-articulatory mapping whereby the phonetic code is accessed (Wilson et al., 
2004). As with many others, this study does not make any particular prediction as to the necessity of this activity for speech perception. Interestingly, this study does make note of reduced activity in the ventral premotor region when the stimulus is a nonspeech sound (Wilson et al., 2004). Given this, the idea that there is some automatic recruitment of the premotor region for the purpose of auditory-to-motor mapping is given some credence. Auditory-to-motor mapping must rely on some gestural repertoire in order to be accessed, non-speech sounds would be less readily recreated, thus weakening recruitment of this region.

Extending the findings from Wilson et al., (2004), Wilson and Iacoboni (2006) examined cortical activation patterns to native and non-native phonemes using fMRI. Across two studies, 12 males and 13 females performed the passive speech perception tasks. The superior temporal gyrus and precentral gyrus were activated when listening to both native and non-native speech signals. Both regions did differentiate between native and non-native phonemes, with a larger activation differential in the superior temporal regions when listening to non-native phonemes. The authors suggested that auditory areas are responsible for translating an acoustic signal to a phonetic code. The motor system contributes by generating candidate phonemic categories. These categories are then compared to acoustic information in the auditory areas. This study highlights that speech perception is neither absolutely sensory, nor motor, but a sensorimotor process.

As stated, while the studies mentioned here suggest that activity is present in the anterior dorsal stream when passively exposed to speech, several studies can be referenced which refute these findings (Belin et al., 2000; Belin et al., 2002; Scott et al., 2009), and others reveal that damage to the anterior dorsal stream has little to no effect on perception performance (Hickok et al., 2011). Despite the passive nature of these studies, it remains possible that at least a portion of this recruitment is related to attention triggered by the presentation of a stimulus. Moreover, fMRI is unable to decompose activity across time, which hinders interpretation. Auditory-to-motor mapping related activity should not occur at any point other than when the stimulus is being presented. The results from these studies suggest that task is a source of variability for anterior dorsal stream recruitment. Other sources may exist, including the sex of the speaker and/or the sex of the participant.

\section{Uneven Participant Distribution and Listener/Speaker Differences}

Many studies across data collection methods (fMRI, EEG, MEG, PET, etc) use unevenly distributed participants between males and females. If speech processing within the dorsal stream is differentiated by sex, including both males and females in a single group increases the potential for incomplete or inaccurate interpretation of data. This section will provide brief overviews of studies which contain uneven sex distributions in their participant pools, and those that may have even representation from males and females but include them in one group for analysis. Finally, this section will cover the few studies which have commented on data which appears to reveal sex-related differences in listening tasks. 
Specifically in speech perception and/or discrimination based tasks, Chevillet and colleagues (2013) used fMRI to examine anterior dorsal stream activity. Of the sixteen participants who performed the tasks, only 7 were females. Taking in to account the evidence from other fMRI research, the functional organization of speech perception may be different between males and females (Gur et al., 2000; Shaywitz et al., 1995), there remains the possibility that the results may be different in the female participants compared to the male participants, though no contrast was performed. This study did employ both a male and female speaker, though the differences in males and females based on the sex of the speaker were not explored.

In a study examining cortical activity related to dominant and secondary language processing, specifically in the laterality of processing, was performed by Centeno et al., (2014). The focus was on the clinical presentation of epilepsy and to examine the utility of fMRI in this population due to atypical representation of language in epileptics (Centeno et al., 2014). The group of participants was 13 males and 3 females. Once again, the laterality of language processing (Gur et al., 2000; Shaywitz et al., 1995) should encourage caution for this line of research, and the effects of epilepsy could further obscure sex-related differences that may exist.

This pattern continues in work examining affective prosody processing over the dorsal and ventral streams. Unsurprisingly, unlike the speech discrimination tasks mentioned throughout this paper, the tasks in this study elicited activity from the right hemisphere analogues to the traditional left lateralized anterior dorsal stream region (Frühholz, Gschwind, \& Grandjean, 2015). Connectivity was assessed across the dorsal stream regions via diffusion tensor imaging (DTI). 3 males and 12 females completed this study, and the resulting data suggests the existence of a similar dorsal pathway in the right hemisphere utilized in affect processing, though the uneven distribution of participants could be, at least partially, responsible for the results.

The uneven distribution of males or females is not limited to the examples briefly described. Other fMRI studies (Bouton et al., 2018; Kandylaki et al., 2016; Meister, Buelte, Staedtgen, Boroojerdi, \& Sparing, 2009), EEG/MEG studies (Bouton et al., 2018; Bowers et al., 2013; Cuellar, Bowers, Harkrider, Wilson, \& Saltuklaroglu, 2012; Jenson et al., 2014; Thornton et al., 2017), PET studies (Peterson, Fox, Posner, Mintum, \& Raichle, 1988), and ERP studies (Liebenthal, Sabri, Beardsley, Mangalathu-Arumana, \& Desai, 2013; Trébuchon, Démonet, Chauvel, \& Liégeois-Chauvel, 2013) suffer from the same potential confound.

Even when studies have the same number of males and females in the participant groups, few studies compare across males and females. Many of the studies are either sex-matched exactly or lean toward more males or females by only one participant, but given the low numbers of overall participants (usually $<15$ ), it is unlikely that between sex group comparisons would be sufficiently powered in order to yield reliable results. Studies such as Ojanen and colleagues (2005) and Kevan and Pammer (2009) have the 
opportunity to examine potential sex related differences within the same task, but no contrasts are reported.

The sex of the speaker is auditory discrimination or passive listening studies presents another possible source of variability that is seldom directly considered. Even studies with the potential to investigate this possibility do not perform further investigation (Chevillet, Jiang, Rauschecker, \& Riesenhuber, 2013). Many of the passive listening or active discrimination studies involving viewing of speech stimuli studies featured a female speaker (Möttönen et al., 2005; Ojanen et al., 2005; Pekkola et al., 2006; Skipper et al., 2005, 2007). However, there are other studies which utilize a male speaker in similar tasks (Jenson et al., 2014; Thornton et al., 2017; Wilson \& Iacoboni, 2006; Wilson et al., 2004). Few studies attempt to approach these short comings specifically in speech perception/discrimination tasks. The results from the following studies do suggest that speaker and listener sex may be important sources of variability to consider.

Bartoli and colleagues (2015) utilized transcranial magnetic stimulation to investigate "speaker-listener distances" and how that altered the recruitment of the anterior dorsal stream region. Twelve participants (6 males/ 6 females) listened to stimuli including recorded speakers intermixed with samples of their own voices. A second test was utilized to test the subjective "differences" between the listener and the speaker. Results from the study indicated that TMS stimulation of the motor effector employed by the speaker to produce the stimulus improved discrimination speech consist with other findings (D'Ausilio, Pulvermüller, et al., 2009). Overall, the findings from this study suggested that interspeaker variability appeared to be causally linked to the level of contribution from the anterior dorsal stream region during syllable discrimination. When rating similarity of listener and speaker, the higher ratings for similarity were found alongside stronger facilitation in discrimination when the tongue region was stimulated. This established a motor gradient, which suggested reduced motor recruitment as distance between listener and speaker increased (Bartoli et al., 2015). This study lays the groundwork for sensorimotor processing differences between males and females, as the listener-speaker differences could be exaggerated by altering the sex of the speaker as well.

Using MEG, Gootjes and colleagues (2006) examined activity in the posterior and anterior dorsal stream regions during a dichotic listening task. Seventeen participants ( 9 females, 8 males) listened to monosyllabic digits spoken by a female speaker and were asked to recall as many digits they could remember from the 'attend' ear, and then from the 'unattended' ear. Accuracy was calculated as a percentage of total. Anterior dorsal stream activity was marked by increased ERS in the alpha band in males which was not present in females. In addition, connectivity between posterior and anterior regions was stronger in males. These results provided support for the possibility of sex differences in brain organization for processing in this particular task. No beta differences were noted, nor was beta even discussed. Alpha differences were also linked to attentional regulation rather than a sensorimotor process, and the authors suggested that alpha oscillation play a "supervisory role" in attentional processes (Gootjes, Bouma, Van Strien, Scheltens, \& 
Stam, 2006). If that is the case, it appears males and females utilize a difference attentional process to complete the same task with similar levels of performance. The importance of this study when considering listener/speaker sex differences arises from a comparison to Thornton et al. (in prep) which used a male speaker to produce stimuli and revealed patterns of ERS in females which were not present in males.

\section{Electroencephalography (EEG) and the Importance of Temporal Sensitivity}

Electrophysiologic and neuromagnetic techniques allow for more time-sensitive investigation of cognitive and sensorimotor processes by revealing patterns of the rhythmic firing of neurons across the cortex. These rhythmic firing patterns, called oscillations, have been divided in to circumscribed frequency ranges and can be attributed to a variety of neural processes (Buzsáki, 2009). Frequency bands include delta $(0.1-2 \mathrm{~Hz})$, theta $(3-7 \mathrm{~Hz})$, alpha $(8-13 \mathrm{~Hz})$, beta $(15-25 \mathrm{~Hz})$, and gamma $(>35 \mathrm{~Hz})$ (Buzsáki, 2009). Significant investigation has occurred in order to identify the role of these oscillatory frequency bands, and what internal and external sources modulate the power of this activity. Broadly, lower frequency oscillatory activity occupies larger regions of the cortex, while higher frequencies are more finely localized to specific regions (Buzsáki, 2009; Schnitzler et al., 2000). Alpha, specifically, is thought to serve multiple roles across the cortex including sensory processing (Bowers et al., 2013; Jenson et al., 2015a), attention (Klimesch, 2012), and gating regional network activity (Jensen \& Mazaheri, 2010; Klimesch, 2012; Zumer et al., 2014). Beta is primarily associated with attention (Frey et al., 2015), working memory (Guntekin and Basar, 2007), and motor processing (Seeber et al., 2014) in a variety of different tasks. Given the links to these cognitive and sensory/motor processes, alpha and beta are of the most interest for further investigation in to the disambiguation of auditory-to-motor mapping and attention and/or working memory.

The purpose of this section is to detail evidence supporting the sensory/cognitive aspects of the alpha band and the motor/cognitive subscribed to the beta band. Following this, the end of the section will detail design considerations to better analyze these processes and pull apart the influence of attention, sensorimotor processing, and working memory from each other. These design considerations informed the selection for both testing conditions and task requirements for the proposed study.

\section{Alpha Oscillations}

Alpha oscillations are found across the cortex, and are thought to be unique in their ability to increase in power through greater synchronization of neuronal firing and decrease through reduced synchronization while other frequency bands appear to reduce in power from an established baseline level (Klimesch, 2012). In broad terms regarding cognitive processes, alpha regulates attention (Hanslmayr et al., 2011) or regionally deactivates irrelevant regions of the cortex in order to route information to task appropriate regions (Jensen and Mazaheri, 2010). 
Attentional allocation via alpha oscillations has been suggested by many studies. Rohenkohl and Nobre (2011) used a visual task paradigm which altered the expectancy of incoming stimuli while measuring alpha band activity via EEG. Twenty participants performed this experiment, with nine males and eleven females. Time between visual stimulus presentations varied between short intervals $(400 \mathrm{~ms})$ and long intervals (800ms). Alpha power desynchronized most strongly in both conditions shortly before the onset of the stimulus. With longer inter-stimulus interval alpha power returned to baseline between stimuli. This did not occur in the shorter inter-stimulus interval condition. Additional alterations to stimulus presentations included irregular (discrete steps from $100 \mathrm{~ms}$ to $700 \mathrm{~ms}$ between stimuli) steps, with no regular interval between stimulus presentations. Without the regularity of stimulus onset, alpha desynchronization was largely absent until stimulus onset. The authors suggest that alpha oscillations modulate cortical excitability which biases perception through the creation of expectation toward incoming information (Rohenkohl \& Nobre, 2011). This draws resources toward information sources and primes the cortical region for processing, thereby allocating attentional resources toward the visual system in this case.

A similar role for alpha oscillations was suggested by Hanslmayr and colleagues (2011). This article suggests that the strength of alpha oscillations are related to likelihood that a stimulus will be perceived or missed. Over several studies it was revealed that higher power in the alpha band, usually across the parieto-occipital regions, was found when participants missed the intended visual stimulus (Hanslmayr et al., 2005; 2007). Conversely, lower pre-stimulus alpha power has been found in trials when the stimulus is more likely to be perceived (van Dijk 2008; Ergenoglu 2004). Hanslmayr and colleagues suggest that ongoing alpha activity is linked to perceptual performance, and that when alpha power is lower, the system is more receptive to incoming stimuli. This could be due to attention allocation toward cortical specific regions for the relevant sensory modality, or simply the result of the waxing and waning of ongoing oscillatory alpha power (Lopes da Silva, 1991) corresponding with the onset of a stimulus.

Allocation of resources toward specific regions of the cortex is the crux of a theory for alpha oscillatory activity provided by Jensen and Mazaheri (2010). The gating-by-inhibition (GBI) theory suggests that alpha power increases in regions of the cortex that are irrelevant to the task at hand. These localized increases in alpha power effectively route information to the specific regions where processing will occur. This theory is attractive in its simplicity, and is supported by several studies.

In studies examining spatial attention, a pattern of activity has arisen supporting the general concepts of GBI. When covert attention is directed toward, for example, the left hemifield alpha power decreases in the contralateral hemisphere which is consistent with active processing of information. In the right hemisphere, however, alpha power increases suggesting deactivation or inhibition of activity within this region (Worden et al., 2006; Rihs et al., 2007; Thut et al., 2006). This pattern of deactivation and activation of certain cortical regions is also seen in visual and motor areas as the task switches to use either process. The alpha power increases in conceivably "unused" portions of the 
cortex are even consistent and robust enough to be implemented as a control signal in some brain-computer interface systems (Salari \& Rose, 2013).

A cross-modal study performed by Fu and colleagues (2001) revealed that this relationship between reduced alpha power in task relevant regions alongside increased alpha power in task irrelevant regions extended to the auditory as well as the visual system. Nine participants were included in this study, of which four were female. Three conditions were presented, auditory alone, visual alone, and audiovisual, each occurred $33 \%$ of the time. Instructions were provided to attend to the signal, and when an audiovisual stimulus was presented, a pre-stimulus click indicated whether the auditory or visual stream was to be attended to. Alpha power reduced in the cortical regions associated with the attended stream, while cortical regions linked to the ignored stream showed increases in alpha power. Thus, alpha appears to ensure that processing resources are not expended in cortical regions that are not relevant.

Critical to this research, this pattern of alpha inhibitory activity has been found in the dorsal and ventral streams of the visual system, suggesting that perhaps the auditory dual streams are susceptible to the same processes. Jokisch and Jensen (2007) instructed participants to remember the identity (ventral) or orientation (dorsal) of visually presented faces. When the task recruited the ventral stream (identity), alpha power increased across the dorsal stream, while the opposite occurred during orientation tasking.

Sensorimotor processing appears to follow similar patterns of activity. Haegens et al., (2010) found that the contralateral primary somatosensory cortex to the hand being used to complete a task is characterized by alpha power decreases. Conversely, the ipsilateral sensorimotor cortex experiences alpha power increases. While the aforementioned studies are not all explicitly indicative of attention, they do make the case for a particular pattern of alpha activity regardless of the process which recruits it. Increases in alpha power occur in disengaged regions, decreases occur in engaged regions (Hanslmayr, Gross, Klimesch, \& Shapiro, 2011).

In addition to simply indicating processing within certain regions of the cortex, alpha suppression is often though to reflect sensory stimulus processing in respective regions of the cortex. Modality specific, or largely specific, regions of the cortex tend to response with reduction in alpha power when a stimulus in that modality is present. This pattern of activity is found in the occipital lobe for the visual system, around the temporal lobe for the auditory system, and the parietal lobe for the somatosensory system.

Mathewson and colleagues (2014) examined alpha oscillations across the occipital lobe during processing of visual stimuli. Sixteen participants (nine women) were presented visual stimuli and were instructed to indicate whether or not they had detected the stimulus. Detected targets revealed alpha suppression before and during target presentation, while undetected targets where characterized by slight alpha enhancement prior to, and reduced suppression during, stimulus presentation (Mathewson et al., 2014). 
This study suggests that ongoing processing of attended and detected stimuli will suppress alpha oscillations in associated sensory regions of the cortex.

Using an audiovisual paradigm in which participant attended to one stimulus stream (auditory or visual) and ignored the other, Gomez-Ramirez and colleagues (2011) investigated alpha oscillatory activity across the cortex. Regional alpha power reductions were found across the cortical region associated with the attended stream, while the ignored stream cortical region was characterized by increases in alpha power. Such findings agree with Mathewson et al., (2014) that alpha suppression in modality specific cortical regions is likely indicative of ongoing processing of sensory information.

Popovich et al., (2010) examined alpha suppression across the somatosensory cortex using median nerve stimulation. This study set out to examine sex-related differences in attentional processes by investigating the alpha band across the somatosensory cortex, well established to index sensory, sensorimotor, and cognitive processes as also highlighted by this section. Critical to this section, whether attention was directed toward or away from the nerve stimulation, alpha suppression occurred. Differences were noted based on sex in what was suggested to be attention related activity by the authors (Popovich et al., 2010), which will be more extensively detailed in a later section of this document. The important note is that alpha activity appears to suppress in response to tactile stimulation in the anterior dorsal stream.

Evidence for the stimulus related suppression of alpha oscillatory activity in the visual and somatosensory systems existed for many years before the discovery of a reliable auditory alpha rhythm with similar response characteristics. While it was long thought to exist, several proposed reasons for its lack of discovery were the relatively small size of the auditory cortex relative to the visual system and the positioning of the core auditory areas (Weisz et al., 2011). In the early 1990s, Tiihonen and colleagues set out to use magnetoencephalography (MEG) to isolate and examine the possible auditory alpha rhythm. Three males listened to $1 \mathrm{kHz}, 80 \mathrm{~dB}, 50 \mathrm{~ms}$ tones presented once every $1.2 \mathrm{~s}$ to each subject's left ear. When the sound was presented, the alpha activity across the temporal region suppressed. Similar suppression was not found in the "eyes open" condition, a well-known condition for suppressing the occipital alpha rhythm. Further still, this rhythm did not suppress in response to tactile stimulation, differentiating itself from the sensorimotor mu rhythm. While the group was relatively small $(n=3)$ for this study, the results were compelling and necessitated further investigation (Tiihonen et al., 1991).

Lehtela and colleagues (1997) was an early investigation in the existence of an auditory alpha rhythm. Using MEG, cortical responses were measured while participants listened to white noise bursts. Of the nine participants, eight revealed patterns of oscillatory activity around 6-9Hz which suppressed when the white noise burst was played. This activity was localized to the superior temporal lobes and provided early support for a distinct auditory alpha rhythm, referred to as the 'tau' rhythm. It remains possible that this suppression is simply a reflection of a cognitive process such as 
attention which results in suppression of modality specific regions, but there is growing evidence for a distinct auditory alpha rhythm.

More recent work has examined auditory alpha in the context of the auditory dorsal stream. Jenson and colleagues recruited 29 right-handed adults to undergo EEG while listening to spoken syllables in quiet and with background noise. Alpha activity was localized to the superior temporal gyrus in the left hemisphere and middle temporal gyrus in the right hemisphere. While not the ideal localization, it was reasonably suspected to be auditory alpha activity. When syllables were presented, alpha activity in the auditory cortex suppressed relative to a pre-stimulus baseline measure. There was little change in auditory alpha activity in the presence of noise or in quiet. Interestingly, the control condition (passive listening to white noise) did not suppress alpha oscillatory activity in the auditory regions. It could be that task requirements were necessary to elicit alpha suppression (cognitive component) or that the suppression was much weaker and thus was relatively minor compared to the greater suppression seen in the two experimental conditions. No explanation was offered by the authors regarding this phenomenon.

\section{Beta Oscillations}

Beta oscillations $(15-25 \mathrm{~Hz})$ are often associated with attention and working memory. This frequency bands seems to be more localized than the broader regional coverage of alpha oscillations. Specifically, beta is most of localized to the left and right premotor and primary motor areas, which has also lead to its association with motor action and observation.

Kaminski and colleagues (2012) extended previous findings from a cat model which had associated beta activity with attentional regulation. Ten participants, of which four were male, listened to and watched stimuli presented following a cue at a random discrete interval of three, five, or seven seconds. Beta power appeared to increase slightly before onset of the stimulus, suggests some attentional mechanism at work in the ongoing monitoring of the scene for relevant information. The authors also suggested that beta increases may serve to organize cortical networks and serve to maintain them, while beta suppression may indicate network reorganization (Kaminski et al., 2012).

Arnal et al. (2012) suggested that beta may play an important role in predictive timing similar to the role suggested by Kaminski and colleagues (2012), but also that beta may serve in the prediction of stimulus content. In a broad review of these processes, the authors suggested that transient increases in beta power (known as beta rebound) follow the rate of presented beats, noting that in the case of omitted beats which would be expected beta power further increases (Arnal, 2012; Arnal et al., 2012). In this case, beta is thought to modulate lower frequency activity in the sensory regions in a top-down manner. Content predictions also modulate beta power. In the presence of external stimulation, beta power initially suppresses which, as noted previously, is indicative of active processing. Predictions of 'what' is thought to similarly be reflected by decreases 
in beta power, often coupled with increases in the gamma band $(>35 \mathrm{~Hz})$ (Arnal et al., 2012). Much like predictive timing, when predictions are violated there is a large increase in the power of beta rebound. Beta is proposed to transmit predictions from anterior regions of the dorsal stream "backward" toward the sensory areas.

Predictive processing, as stated, takes the forms of predictive timing or content predictions. It is possible that predictive timing is characterized by increases in beta power (Kaminski et al., 2012), though similarly, reductions in beta power have been associated with predictive timing as well (Fujioka et al., 2012; 2015). While attentional processes such as prediction generation, regardless of timing or content, have been shown to modulate beta power, as have working memory processes. Several studies have revealed reductions in beta power (suppression) which coincide with likely encoding of stimuli in working memory for later use for a particular task.

Lundqvist et al., (2015) revealed a pattern of beta and gamma activity in working memory tasks using non-human primates. Using two monkeys trained to retain multiple colored squares in working memory, patterns of oscillatory activity were investigated during the training period while the locations and colors of the visually presented squares were encoded. Shortly following $(<300 \mathrm{~ms})$ training stimulus onset, beta power decreased. This was paired with an increase in gamma power. These power change "bursts" appeared to increase in duration and power slightly as working memory demands increased through the addition of another colors/positions to remember. The authors suggest that activity, attributed to both beta and gamma modulations, and is due to stimulus encoding later use. Similar decreases in power of the beta band appear during the recall portion of the task, indicating that accessing working memory may rely on the same processing as initial encoding. The authors make note of the roles of other oscillatory frequencies, suggesting that slower oscillations (i.e. alpha) may also be important for working memory (Lundqvist et al., 2015).

Chen and Huang (2016) used EEG to examine oscillatory activity during a visual n-back task in eighteen adults. N-back tasks are commonly used to tax working memory, and require matching presented stimuli to previous stimuli ( $n$ times) before the current presentation (ex 2-back: / $\underline{\mathrm{a}} / / \mathrm{e} / / \mathrm{a} /$ ). Relative to the 1-back condition, beta spectral power increased during the 2-back condition across the right-central electrode locations. Unsurprisingly, alpha power decreased across the occipital lobe, an expected finding since the stimulus was visual and power suppression is generally indicative of stimulus processing. The authors suggested that relative increases in beta power during the 2-back condition is indicative of beta is responsible for active maintenance and temporal duration.

Expanding the role of beta in cognitive processes is an ongoing pursuit. The expansion to these cognitive processes occurred as the response patterns of beta to varying degrees of movement became more nuanced. Given the nature of speech perception and the involvement of cognitive and sensorimotor processes, it is necessary to examine the situations in which beta responds to motor/sensorimotor demands. 
Using high density EEG, Seeber and colleagues (2014) examined both beta and alpha oscillations during upright walking and standing in ten participants. Relative to standing, upright walking resulted in suppression of both beta and alpha across the sensorimotor regions of the cortex. The suppression patterns were explained as representative of a movement related change to the state of the system regarding cortical excitability (Seeber, Scherer, Wagner, Solis-Escalante, \& Muller-Putz, 2014). Given other interpretations of oscillatory power suppression suggesting that power reductions indicate active processing, it is likely then, that this revealed pattern of suppression represents active motor processing of overt movements.

Observation of action has also been shown to suppress beta oscillations across the sensorimotor/primary motor regions of the cortex. Twenty adults viewed an actor interacting with several objects while EEG tracings were recorded. One group of observers was familiar with the weight of the objects, one group was given brief exposure to the objects, and the third group had no experience with the objects on screen.

Suppression of beta and alpha was strongest during action observation by the group with experience handling the objects on screen, however, all groups revealed these patterns of suppression (Quandt \& Marshall, 2014). The authors indicate that prior experience with objects alter the sensorimotor processing associated with action observation. Such a finding is similar to other work which suggest that biological relevance increases reactivity of both beta and alpha oscillations (Thornton et al., 2017; Fox et al., 2016). It is reasonable to suggest, then, that previous experience with objects could increase the biological relevance of the object when viewed.

De Lange and colleagues (2008) added that beta oscillatory activity across the frontal regions of the cortex is suppressed by imagery of motor actions, adding to the overt and observed conditions previously mentioned. Participants viewed rotating line drawings of hands moving and were instructed to indicate whether it was a left or right hand. Beta suppression was revealed across the precentral gyrus, when a right hand was viewed, suppression was stronger over the left hemisphere and vice versa. As imagined movements became more complex, degree of power changes in beta increased, suggesting that increased complexity further recruits motor processing. Thus, beta suppression appears to be linked to motor processing whether the action is being performed, observed, or imagined. The difference is the degree to which beta suppresses.

\section{The Mu Rhythm}

Taken together, alpha and beta across the anterior dorsal stream have been described as the sensorimotor mu rhythm (Bowers et al., 2013; Fox et al., 2016; Hobson \& Bishop, 2016; Jenson et al., 2014; Kittilstved et al., 2018; Thornton et al., 2017), expanding upon the previous definition of the mu rhythm which focused purely on alpha reactivity and considered beta to be highly correlated (Carlqvist, Nikulin, Stromberg, \& Brismar, 2005). 
Mu rhythm reactivity has been measured in response to many different stimulus paradigms and modalities. Broadly, the mu rhythm has been defined as responsive to stimuli with 'biological relevance'(Cuellar \& del Toro, 2017; Marshall \& Meltzoff, 2012; Minichino, Singh, Pineda, Friederich, \& Cadenhead, 2016; Pani et al., 2014; Schnitzler et al., 2000). 'Biological relevance' is a fairly loosely defined term which indicates that the sensorimotor mu rhythm with respond to stimuli that carry information that carries some meaning to the individual experiencing the stimuli. For example, a video of someone kicking a ball is biologically relevant, as the viewer can understand the action, the cause, the effect, and possibly the intention. In contrast, a random assortment of moving lights is not biologically relevant, as it carries with it not structure, action, cause, effect, or intent.

Visual stimuli are often used to investigate mu rhythm reactivity. Mu rhythm suppression has been noted in response to observation of movements (Cannon et al., 2014; Drew, Quandt, \& Marshall, 2015; Quandt \& Marshall, 2014; Rüther, Brown, Klepp, \& Bellebaum, 2014), painful scenarios (Yang, Decety, Lee, Chen, \& Cheng, 2009), speech (Crawcour, Bowers, Harkrider, \& Saltuklaroglu, 2009), and reading (Hobson \& Bishop, 2017). Each of these stimuli vary in the degree of 'biological relevance', but all maintain it to at least some degree.

Specifically, the series of studies from Drew and colleagues (2015) and Quandt and Marshall (2014) found that mu suppression in observation of movement is linked to experience with the observed action. Quandt and Marshall (2014) examined this by allowing groups of participants to have varying degrees of experience with objects that they were to observe being interacted with. One group was allowed to lift, move, and otherwise interact with the objects for an extended period, another was allowed to examine and interact with the objects for a brief period, and the last was provided only written information about the objects. For the groups who experienced the objects, mu suppression was differentiated by the weight of the object, while those whose only experience with the object was through reading, such differentiation did not exist. The authors suggested that even limited experience with an object alters sensorimotor processing when viewing interactions with the object. This study revealed not only the sensitivity of the mu rhythm to observed movement, but also the sensitivity of the mu rhythm to influence from previous information and experience.

Yang and colleagues (2009) examined mu reactivity in response to visually presented painful and non-painful situations. Mu suppression was greater in situations that were 'painful' (i.e. a finger about to get cut by scissors) compared to non-painful situations (i.e. a finger not about to get cut by scissors). In addition to this finding, females showed stronger mu suppression than males in the painful situation. Not limited to 'biological relevance', the authors suggest the findings indicate that the mu rhythm may be a biomarker for empathic mimicry, and that females may reveal stronger empathic mimicry than males.

Speech signals presented visually have also resulted in significant mu suppression, relative to noise and a visually presented kaleidoscope image (Crawcour et al., 2009). Interestingly, speech presented via audition did not result in significant levels 
of mu suppression. Broadly, the authors suggested that while visually presented speech was sufficient to recruit anterior dorsal stream activity, the task was not sufficiently complex to recruit the same region for audition. The lack of mu suppression in response to auditory presentation of speech signals has been contradicted by other studies, which have found mu suppression in response to auditory signals in a variety of different stimulus contexts.

A wide array of studies have utilized auditory stimuli to investigate mu rhythm reactivity. While many are detailed later in this chapter, it is useful to discuss a few here that reveal that even non-speech stimuli that have revealed mu rhythm reactivity, suggesting that speech isn't necessary to satisfy 'biological relevance', though it is an ideal stimulus to utilize. Stimuli including piano melodies (Wu, Hamm, Lim, \& Kirk, 2016), tone sequences (Thornton et al., 2017), and action sounds (Pineda et al., 2013) have elicited mu rhythm response.

Using a group of trained pianists, Wu and colleagues (2016) investigated mu suppression in these participants while passively listening to piano melodies. Relative to rest, mu suppression was found in both the left and right hemisphere and was broadly localized to the anterior dorsal stream, consistent with other findings (e.g. Muthukumaraswamy, Johnson, Gaetz, \& Cheyne, 2004). This study suggested that due to training, these pianists associated what may generally be considered non-biologically relevant stimuli (melodies) to biologically relevant motor actions, as these participants were likely able to recreate the perceived stimuli. This study also reveals that even when stimuli are only presented via audition, mu suppression can occur.

Thornton et al. (2017) investigated the mu rhythm using speech and non-speech segmentation tasks via aurally presented stimuli. Regardless of speech content, all conditions requiring discrimination revealed mu rhythm suppression. However, mu suppression, particularly in beta, was stronger when speech signals were presented relative to the non-speech conditions. Interestingly, segmentation tasks actually enhanced mu rhythm power following offset of the initial stimulus when compared to nonsegmentation tasks. This study reveals that increased cognitive demands can influence mu reactivity in addition to stimulus content.

Pineda and colleagues (2013) examined the mu rhythm using hand or mouth action sounds, environmental non-action sounds, and control sounds. Mu suppression was found to be greater in response to the action based sounds relative to control sounds, particularly over the left hemisphere. Hand-based actions also revealed increased suppression over the hand area compared to the mouth-based action sounds. Additionally, mu suppression was greater to non-action sounds relative to control sounds over the left hemisphere. Overall, the study suggests that effector specific sounds may preferentially recruit effector specific regions of the cortex, which is indicative of specific auditorymotor processing when biologically relevant stimuli are perceived and processed. 


\section{Event-related Design}

Alpha and beta are linked to the sensory, motor, and cognitive demands imposed by a task. The challenge is establishing the nature of the processes at work in an attempt to pull cognition from sensory and motor evaluation. Event-related EEG designs are employed to examine oscillatory activity before, during, and after the onset/offset of a stimulus. This allows for more fine-grained analysis of oscillatory power fluctuations. In this context, event-related synchronization (ERS) is a transient increase in power within an oscillatory frequency compared to a recorded baseline measure. ERS is generally associated with inhibition of processes, as indicated by evidence from the alpha band. Conversely, event-related desynchronization (ERD) is indicative of a transient decrease in oscillatory power from the same pre-established baseline measure (Pfurtscheller \& Lopes, 1999).

Examining EEG/MEG data in reference to the onset of a stimulus should allow for activity to be more carefully characterized. Activity before stimulus onset should be related to attention, while activity after should be linked to working memory processes. Activity during stimulus presentation is slightly more complex. It could be representative of ongoing attention or working memory consolidation, but given theoretical models of speech perception in particular, it could also be related to auditory-to-motor mapping. Auditory-to-motor mapping is a sensorimotor process, and sensorimotor processes underlie many disorders. Understanding sensorimotor processes in average populations will inform potential identification of aberrant activity in various clinical populations.

\section{Auditory-to-Motor Mapping and Sensorimotor Processing}

The longstanding recruitment of motor regions when processing speech signals has been linked to the process of auditory-to-motor mapping. This processing represents a unifying code for sensory and motor information, an idea suggested by Prinz (1997). Using sensory information to guide motor action classically defines sensorimotor activity, establishing auditory-to-motor mapping as a sensorimotor process.

Auditory-to-motor mapping is defined as the translation of auditory stimuli on to the articulatory gestures of which the listener would utilize to recreate these sounds. This process is thought to overcome the massive variability in speakers. Given that speaker variability includes speaker sex, frequency, rate, and various other dimensions, mapping sounds to the gestures which would create the presents a compelling method for overcoming the lack of invariance problem.

Fowler and colleagues (2003) investigated this auditory-motor mapping process using two testing conditions. In both test conditions, participants listened to vowelconsonant vowel (VCV) productions where the onset vowel had unpredictable durations. In the first condition, participants listened to and watched a speaker. When the speaker shifted to producing the CV portion of the VCV listeners were asked to produce a specific CV which did not match the speaker's production. In the second condition, the 
task was the same with the shift of the participant instead matching the speaker rather than adhering to one production. The study measured the "onset of closure" of the participant's CV production in each condition. The first condition was faster than the second condition by $26 \mathrm{~ms}$. The authors indicate that this minor latency difference suggests that even though the second task was slower, it is indicative of access to speech articulatory gestures extracted from the signal and mapped to internal gestures (Fowler et al., 2003). This conclusion was drawn as the difference between the conditions was much smaller than the condition latencies were proposed to be by Luce (1986).

The close inter-relationship of perception and production, and thus auditory-tomotor mapping has been explained in feedback and feedforward models. Hypotheses about incoming signals that are mapped onto to motor commands used in production are extracted from sensory input and extend from sensory to motor regions. Past experience is thought to inform these motor actions which are then used to predict the somatosensory and acoustic results of corresponding motor action. This "forward model" extends from anterior motor/premotor regions toward sensory regions and are thought to "weight" the interpretation of sensory input toward the sound associated with the production method (Skipper et al., 2007).

\section{Measurement of Sensorimotor and Cognitive Processes via EEG and Sex-related Differences}

Pursuant to cognitive and sensorimotor processing, investigation of a particular oscillatory rhythm is commonly employed. This rhythm, known as the sensorimotor mu rhythm, is characterized by peaks of oscillatory activity in the alpha and beta frequency bands across the premotor cortex, primary motor cortex, and primary somatosensory cortex (Bowers et al., 2013; Jenson et al., 2014; Matsumoto et al., 2010; Moore, Gorodnitsky, \& Pineda, 2012; Pfurtscheller, Neuper, Andrew, \& Edlinger, 1997; Schnitzler et al., 2000). Within the mu rhythm, alpha has been associated with sensory processing (Jones et al., 2009; Saltuklaroglu et al., 2017), analysis (Jenson et al., 2014), or attention (Hanslmayr et al., 2011; Klimesch, 2012), while beta is thought to reflect attention (Gola, Magnuski, Szumska, \& Wróbel, 2013; Sacchet et al., 2015), auditory to motor mapping (Thornton et al., 2017), or working memory (Jenson et al., 2014; Thornton et al., 2017), suggesting that each oscillatory band may cooperate to perform distinct functions in cognitive and sensorimotor processes (Brinkman, Stolk, Dijkerman, de Lange, \& Toni, 2014).

Alpha appears to respond to sensory information in the visual (Crawcour, Bowers, Harkrider, \& Saltuklaroglu, 2009; Cuellar et al., 2016), auditory (Bowers et al., 2013; Thornton et al., 2017), and somatosensory (Popovich et al., 2010) modalities with a reduction in power relative to a baseline measure, which suggests an increase in processing within these regions of the cortex (Graimann \& Pfurtscheller, 2006). Crawcour et al., (2009) used visually presented syllabic stimuli and revealed reduction in alpha power. Similarly, Cuellar utilized visual presentation of an animated video of swallowing, and revealed suppression of alpha power. Bowers et al., (2013) and Thornton 
et al., (2017 each used auditory stimuli to elicit suppression of alpha power. The suppression of alpha power occurred regardless of stimulus content, as both syllable speech and non-speech (tones) were used. Median nerve stimulation also suppresses alpha across the somatosensory cortex, regardless of directed attention to the stimulation (Popovich et al., 2010). Together, it appears that alpha within the mu rhythm responds to sensory stimulation regardless of the modality employed to elicit the response.

Beta is not traditionally associated with the mu rhythm or is largely ignored due to a proposed strong correlation with alpha activity (Carlqvist, Nikulin, Strömberg, \& Brismar, 2005). However, it is important to note that beta does appear to respond to different stimulation, mostly involving movement. Quandt et al. (2012) found that beta power suppresses in response to both observed object interaction and actual object interaction in addition to alpha power suppression. Strength of beta suppression appears to be sensitive to previous experience with objects and qualities including weight (Quandt $\&$ Marshall, 2014) and even responds prior to experiencing the weight of an object based on that previous experience. Seeber and colleagues (2014) further investigated sensorimotor oscillatory activity during walking and standing. Beta suppression increased when walking compared to upright standing, suggesting that overt actions are sufficient, but not necessary when considering evidence from Quandt et al., (2012), to elicit beta suppression (Seeber et al., 2014).

Based on the stimuli presented across these studies, a pattern emerges. The stimuli (or actions) are generally characterized by biological relevance. This biological relevance has led to the suggestion that the mu rhythm is a measure of mirror neuron activity (Fox et al., 2016), and that biological relevance is a necessary factor to elicit power fluctuations in the mu rhythm. However, alpha and beta fluctuations across mu generating regions have been found in cognitive tasks that may not be explicitly sensorimotor in nature.

In a working memory task, where the number of items held in working memory was varied systematically, alpha power was found to increase alongside increasing working memory load (Jensen, Gelfand, Kounios, \& Lisman, 2002). This increase was found directly across the somato-motor cortex, consistent with regions traditionally associated with the mu rhythm. Alpha has been shown in increase in power in the presence of a distractor stimulus (Bonnefond \& Jensen, 2012), suggesting that the role of alpha may not be limited to sensory analysis or even in working memory maintenance, but may also serve to protect working memory or sensory analysis from competing streams of information (Bonnefond \& Jensen, 2012). Additionally, alpha has been shown to increase in regions of the cortex unassociated with presented stimuli, suggesting that regional increases in alpha power from baseline may indicate unused or inhibited regions of the cortex (Jensen \& Mazaheri, 2010; Knyazev, 2007; Murphy, Foxe, Peters, \& Molholm, 2014).

Beta has also been implicated in working memory, where decreases in beta power are associated with maintenance of relevant information necessary for task completion (Jenson et al., 2014; Thornton et al., 2017), a link to the phonological working memory 
loop suggested by Baddeley (2012). Beta power decreases have also been associated with prediction about the content of upcoming stimuli (Arnal et al., 2012), the timing of upcoming stimuli (Arnal, 2012; Fujioka, Ross, \& Trainor, 2015), or simple attention toward upcoming information (van Ede, de Lange, Jensen, \& Maris, 2011). As shown by Quandt and Marshall (2014), beta has some predictive capability, as even in the absence of interaction with an object, beta suppresses in response the expected weight of an object that has been previously handled.

Clearly, alpha and beta oscillations within the mu rhythm respond to sensory and motor stimuli but are also sensitive to cognitive demands associated with tasks that are not necessarily sensorimotor in nature. Given this, it is clear the mu rhythm is an ideal source of information regarding these processes and may allow for investigation of sexrelated differences in cognitive or sensorimotor processing. The mu rhythm has been shown to be sensitive to sex-related differences, however these studies are primarily limited to mu alpha.

In visual perception of pain in others, Yang and colleagues (2009) revealed that females experience greater mu alpha suppression than males in response to painful appearing scenarios. Males did not differ in suppression between painful and non-painful scenes. While interpreted as a measure of mirror neuron activity and empathic mimicry, the authors suggested that females may be more empathic, and thus exhibit more processing when experience in others. It appears that pain empathy elicits a sensorimotor response, and that the sensorimotor mu rhythm is sensitive to sex-related differences.

Examining directed attention, Popovich et al., (2010) measured mu rhythm reactivity over time when attending to, or ignoring, median nerve stimulation (MNS). No differences were noted in the degree of mu-alpha suppression between males and females, but the length of alpha suppression was greater in females compared to males when attention was directed to the somatosensory stimulus. These results were taken to suggest differentially attention related processing strategies between males and females. Specifically, females were taken to employ a more effortful, top-down mediated strategy to direct attention to the stimulus and keep attention there. Male activation patterns were attributed to a more bottom-up processing strategy.

Cheng and colleagues (2006) utilized MEG to investigate sex-related differences in mu beta during perception of hand movement compared to moving dots. Females had greater mu suppression for the hand movement compared to the dots as well as compared to males. Interestingly, males revealed a reversed pattern of mu beta suppression, with greater suppression to moving dots compared to hand movements. It was suggested that this could underlie a male prevalence in autism, as the biologically relevant stimulus (hand movement) elicited less of a response in males and autism is characterized by reduced empathizing and increased systematizing, beyond what is often seen in typical populations (Yawei Cheng et al., 2006).

Given support for the sensitivity of the mu rhythm to sex-related differences, and the ability to reflect differential processing related to sensorimotor and/or cognitive 
processing, further investigation is necessary to characterize the degree to which sexrelated mu rhythm differences extend in to other areas of sensorimotor and cognitive tasks. A variety of speech perception and discrimination tasks have been shown to elicit mu rhythm fluctuations in both alpha and beta, including sex-related differences.

\section{Sensorimotor Activity in Speech Perception}

Speech perception tasks are ideal for investigating the possibility of sex-related differences in sensorimotor processes as they have been shown to elicit patterns of alpha ERS and ERD, as well as beta ERD. To this end, several studies have utilized speech perception tasks in order to investigate and establish these patterns of what would be considered typical sensorimotor processing. However, speech perception recruits common cognitive processes as mentioned within the same cortical regions, including attention and working memory. This makes speech perception tasks ideal for investigation of these processes concurrently. These studies started by evaluation fluctuations in alpha power using a continuous recording, but progressed in to more finegrained temporal investigation of both alpha and beta activity using independent component analysis (ICA) and event-related spectral perturbations (ERSPs) with denser EEG electrode arrays.

Crawcour et al. (2009) started by investigating alpha suppression across channels $\mathrm{Cz}, \mathrm{C} 3$, and C4. Fourteen participants (all female) watched and listened to audiovisual stimuli which consisted of speech, noise, or pure tone auditory information combined with speech, noise, or kaleidoscopic visual information. Noise-noise was used as a baseline condition. All visual stimuli resulted in mu-alpha suppression relative to this baseline measure, while none of the non-speech or speech conditions suppressed the mualpha when the signal was only presented aurally. The results were thought to suggest that visual stimuli appear to be more closely associated with motor activity than purely acoustic stimulation (Crawcour et al., 2009). While several studies have revealed motor regional activation to purely acoustic stimulation (Watson et al., 2004; Pulvermuller et al., 2006), this study did not. It was thought that the reduced task demands were not sufficient to elicit anterior motor activity.

Cuellar and colleagues (2012) extended this idea by introducing increased task demands via phoneme level segmentation requirements. Phoneme level segmentation is the separation of onset or offset phonemes from the constituent word as defined by a series of fMRI studies from Burton and colleagues (Burton, 2001; Burton \& Small, 2006; Burton et al., 2000; LoCasto et al., 2004). Ten adult females participated in the first portion of this study, which involved listening for a target pseudoword. The three conditions included identification, discrimination, and discrimination in a noisy background. In this experiment, discrimination in noise was the only task which elicited significant mu-alpha suppression. The second experiment introduced the aforementioned segmentation process of both speech and non-speech stimuli. Suppression of alpha across electrodes $\mathrm{C} 3, \mathrm{Cz}$, and $\mathrm{C} 4$ was greater in speech conditions compared to tone conditions. The speech segmentation task only elicited greater mu-alpha suppression over $\mathrm{C} 3$, and 
only relative to the baseline condition. The authors interpreted these results as indicative of mu-alpha sensitivity to auditory signal processing in the anterior dorsal stream. In addition, it appears that suppression is associated with more difficult tasks and more sensitive to linguistic rather than non-linguistic (tonal) information.

The previous works were extended by Bowers et al. (2013) by using independent component analysis (ICA) to isolate mu rhythm fluctuations from ongoing oscillatory activity. The main focus of this study was to provide support for a theoretical model (constructivist/analysis-by-synthesis) which suggests that articulatory hypotheses may be generated in order to enhance perception by narrowing analysis of incoming signals to a more limited set of possibilities. Sixteen (15 females/1 male) participants passively listened to, or discriminated speech and non-speech stimuli. Conditions were also marked with white background noise. Chance level SNR and high accuracy SNR were used to examine mu rhythm activity for fluctuations based on the performance of the participant. No differences from the baseline measure employed for time-frequency analysis were found in the passive listening task. For the left mu cluster, beta suppression was found in correct speech discrimination trials relative to the chance performance condition. These results were supportive of the generation of early forward models which are used to compare to incoming sensory stimuli, but only when a task was involved as no passive differences were noted.

Jenson et al. (2014) examined the mu rhythm in both speech discrimination and speech perception tasks. Twenty participants ( 17 females, 3 males) were recruited to perform six different tasks. These tasks were passive listening to white noise, discrimination of speech in quiet, speech discrimination in noise (4dB SNR), overt and covert syllable production, and three syllable word production. Time-frequency analysis was performed on ICA produced mu rhythm components. The production conditions were characterized by suppression of both mu alpha and mu beta, consistent with motor processing even when actions are imagined. The suppression, however, was much greater in the overt production conditions. The discrimination conditions revealed early alpha ERS paired with beta ERD both just before and during stimulation. While not statistically significant, the noisy discrimination appears to have more robust inhibitory alpha activity. These data were interpreted as indicative of hypothesis generations and testing, as they are similar to the findings from Bowers and colleagues. It was also suggested that the additional buildup of alpha ERS in the noise condition may represent an effort to limit the influence of extraneous noise on processing of the intended signal.

Further investigation in to segmentation based tasks was performed using ICA and time-frequency analysis of mu rhythm components (Thornton et al., 2017). This study used the tasks specified in work from Burton and colleagues modified to fit an event-related EEG design. These tasks included passive listening to white noise, segmentation of initial phonemes from whole pseudowords for the purpose of discrimination, phoneme discrimination in rhyming pseudowords, segmentation of initial sounds in a 3-tone sequence, and discrimination of tones. Unlike results from Bowers et al. (2013) and Jenson et al. (2014), no early alpha ERS was noted in any conditions, however, both segmentation conditions were characterized by alpha ERS at the offset of 
the first stimulus present in a pair. Tone conditions reveal alpha ERD at stimulus onset with paired beta ERD. Early alpha ERD in the speech conditions coincided with early beta ERD. When comparing tone conditions with speech conditions, beta ERD and the strength of the alpha ERD differed. When comparing grouped segmentations compared to non-segmentation conditions. Segmentation conditions were marked by bursts of alpha ERS following initial stimulus offset. This finding was consistent with previous studies looking specifically at segmentation tasks (Shahin \& Pitt, 2012). Unlike the studies investigation speech perception in this section, no early stimulus alpha ERS was noted. Previous works explained such ERS as narrowing of sensory analysis or a sensory gating method. Thornton et al. (2017) explained this lack of consistency as related to available set size for stimuli combinations, as this study featured four times more combinations which was suggested to be too many possible iterations for effective hypothesis generation and as no noise was presented, the need for gating was limited. When hypotheses are generated but deemed ineffective, continued generation ceases (Kleinsorge \& Scheil, 2016).

Given the strong link between sensorimotor processes and many speech, language, and hearing disorders, Saltuklaroglu et al. (2017) investigated sensorimotor speech processing in a group of adults who stutter (AWS) and matched counterparts (TFS). This study employed time-frequency analysis on EEG data recorded during active discrimination of tones and speech, in both quiet and noisy backgrounds. The control task used was passive listening to white noise. Fifty-four age and handedness matched participants (27 AWS, 27 TFS) completed the study. ICA/time-frequency analyses were performed to isolate and analyze mu rhythm components for each group. Spectral analysis was also performed and revealed reduced beta power in all across conditions. Time-frequency differences were found only in conditions with noise. The PWS group was characterized by increased beta ERD while listening to noise and during noise backed discrimination conditions only. In addition to greater beta ERD, the PWS group was also marked by reduced alpha ERS relative to the TFS group. The results were interpreted as indicative of reduced forward modeling capability as well as reduced sensory gating. It is possible that the early activity differences may reflect utilization of a difference attentional process. In most conditions, activity after stimulus offset, often attributed to working memory, did not differ between groups. Interestingly, this study also found a behavioral performance difference only in the tone discrimination in quiet condition. The findings from this study compared to those of Jenson and colleagues (2014) and Bowers et al. (2013) suggested that there may be sex-related difference in sensorimotor processing for speech as this study had primarily male participants while the two previous works featured primarily females.

Findings from the majority of these studies suggested that sex-based differences in speech perception may be revealed through investigation of mu rhythm fluctuations over time related to the processing of speech (Thornton et al., in prep). Twenty one males and twenty one females listened to and discriminated syllables in both quiet and a noisy background. Though performance of the task did not differ between sexes, timefrequency analysis of ICA derived mu rhythm components revealed sex-related differences in both mu alpha and beta before and during stimulus presentation. In quiet 
speech discrimination, females were characterized by pre-stimulus alpha ERS which extended in to stimulus presentation. Males revealed pre-stimulus mu-beta ERD with alpha ERD arising shortly after stimulus onset. Both males and females were characterized by paired alpha ERD and beta ERD following stimulus offset reliably indicated in other works as working memory and/or covert rehearsal (Bowers et al., 2013; Jenson et al., 2014; Saltuklaroglu et al., 2017). In a noisy background, however, patterns of activity in females did not change, and a slight, but non-significant, increase in alpha ERS was noted. Patterns of activity in males did change, pre-stimulus alpha ERS appeared and did significantly differ from activity in males during quiet speech discrimination. This change in males suggested that the impact of background noise is different in males and females. There were no significant differences in the noisy condition between sexes. The results were interpreted to suggest the presence of a differential recruitment of attentional processes in this speech discrimination task. Males utilized more beta band processing suggestive of additional motor recruitment or attention to timing (Fujioka et al., 2015), while females utilized activity which appeared to reflect hypothesis and test methodology (Bowers et al., 2013). Though other studies have shown sex-related differences in these regions of the cortex, they did not employ speech signals (Cheng et al., 2009; Cheng et al., 2008; Popovich et al., 2010).

Oliveira et al. (in prep) examined the sensorimotor mu rhythm when participants discriminated pairs of native and pairs of non-native sounds. In addition to active discrimination, participants passively listened to the same syllables. The goal of this study was to examine the mu rhythm in these conditions to compare the effects of nonnative speech to native speech both in active discrimination to address general cognitive processes while passive conditions were designed to investigate automatic recruitment of the anterior dorsal stream in the presence of speech signals. Suppression of both alpha and beta were greater during active discrimination of native syllables compared to nonnative syllables. Passive listening followed the same pattern albeit with weaker patterns overall compared to the active discrimination tasks. Post-stimulus activity in the active discriminations was characterized by alpha and beta ERD regardless of stimulus test, reinforcing findings associated with working memory. Passive listening tasks revealed slight alpha/beta ERD in the native syllables during stimulus presentation, while nonnative syllables elicited slight alpha ERS and little to no change in patterns of beta activity. The activity in passive listening was explained as automatic recruitment of the anterior dorsal stream associated with template matching. Templates are more easily matched when such templates exist, as would be expected with native sounds. The results from this study alongside the findings from Thornton et al. (in prep) provide support for the idea that sex-differences in speech processing exist, and that these differences may extend to passive listening tasks.

\section{The Auditory Cortex and Sex-related Differences}

As noted, the auditory dorsal stream is made up of a network of regions and is not limited to the premotor cortical regions. Other nodes include the inferior parietal lobe and the auditory cortex. Some models of dorsal stream dynamics suggest that information is 
acquired from stimuli in the auditory regions of the cortex and is compared to hypotheses initial generated in the anterior dorsal stream (Skipper et al., 2005), while others suggest auditory cortical activity precedes any activation of the anterior dorsal stream (Fowler et al., 2003). Network communication in the auditory dorsal stream may not only be influence by sex in anterior regions, but the posterior region as well. The patterns of activity revealed by analysis of characteristics in each region of the cortex will provide information regarding current theoretical models for speech perception and may reveal differential patterns of activity in multiple regions of the cortex based on the sex of the listener.

Unlike the anterior dorsal stream, the posterior portion is characterized by fluctuations primarily in alpha activity. Weisz and colleagues (2011) examined evidence regarding the presence of an independent alpha rhythm site across the auditory cortex, given previous findings for alpha sources across the sensorimotor and occipital regions of the cortex. Several early studies suggested the presence of an auditory source of alpha oscillations, tentatively referred to as the 'Tau' rhythm, which respond only to auditory stimuli, not visual or tactile (Lehtelä, Salmelin, \& Hari, 1997; Niedermeyer, 1990; Tiihonen et al., 1991). Using MEG, Tiihonen et al. (1991) identified an auditory alpha rhythm when three participants listened to $1 \mathrm{kHz} 50 \mathrm{~ms}$ square wave tones. This study tested participants both with eyes open and eyes closed, to which the alpha rhythm did not differ. Limited additional detail was provided, as this was a preliminary study to identify an alpha wave that was unresponsive to visual and sensorimotor stimuli.

Lehtela and colleagues' study revealed transient suppression of the auditory alpha rhythm, measured at 6.5 to $9.5 \mathrm{~Hz}$ in 8 of the 9 participants. The cortical signal was recorded via neuromagnetometer, during the presentation of $500 \mathrm{~ms}$ bursts of white noise to each ear independently. When the stimulus was presented to the right ear alpha suppression was similar in each hemisphere, however, while the stimulus was presented to the left ear suppression was much greater of the right hemisphere. The results indicated the presence of a generator site for an alpha rhythm across the supratemporal areas and may even be right hemisphere dominant. This rhythm does appear to suppress in the presence of auditory stimuli at even the most basic level.

The review previously mentioned the presence of the auditory alpha ('tau') rhythm and provided evidence for its utility in both the research and future clinical implementation (Weisz et al., 2011). The skeptical reception by some to the presence of the auditory alpha appears likely to be due to its predisposition to being obscured by stronger alpha rhythms present in the visual and sensorimotor regions. However, as localization of neuromagnetic and electrophysiological signals improves, the ability to investigate the auditory alpha rhythm should improve and provide more sensitive information regarding auditory processing. Interestingly, and perhaps an important note in the context of the auditory dorsal stream, limited evidence has been found suggesting that beta may also suppress across the auditory cortex during sensory perception as well (Lehtelä et al., 1997). Future directions presented in this review (Weisz et al., 2011) suggested examining alpha oscillations across the auditory regions for clinical conditions such as tinnitus, which has been picked up by many studies (De Ridder, Congedo, \& 
Vanneste, 2015; De Ridder, Vanneste, Langguth, \& Llinas, 2015; Leaver, SeydellGreenwald, \& Rauschecker, 2016; Meyer, Luethi, Neff, Langer, \& Buchi, 2014; Vanneste, Song, \& De Ridder, 2013; Vanneste, Van DeHeyning, \& DeRidder, 2015).

In a speech perception task, twelve males and twelve female participants listened to words with varying degrees of comprehensibility obtained via vocoding with different numbers of spectral bands. The task was to indicate degree of comprehension with a 1-4 rating scale. Localization of EEG activity during this task revealed stronger areas over the parietal/temporal regions for the alpha band, and over the prefrontal regions in the theta band. Across the temporal regions, alpha suppression increased as the amount of spectral bands increased, with the most suppression seen in the 16-band spectral information condition. Overall, these results were interpreted as representing a close link between comprehensibility of a speech signal and the suppression of alpha across the auditory cortex (Obleser \& Weisz, 2012). Once again, beta suppression begins to appear was spectral information increases, though this note is not approached but could be interpreted as an indication of emerging communication with the anterior dorsal stream, and given the network established in speech discrimination tasks, this does appear to be logical. However, no connectivity measures were utilized, and activation of the anterior dorsal stream was not found. Instead, activity was found in the prefrontal cortex.

In a continuing effort to investigate auditory alpha dynamics under varying conditions, a well-known phenomenon found in the visual system was tested in the auditory system. Top-down processing can influence activity in sensory regions of the cortex due to anticipation of sensory input. When just listening, with no anticipatory prompt, alpha suppression occurring the both auditory cortices, beginning shortly following stimulus onset. In the attend conditions, in which a visual cue instructed participants as to which ear was to be attended, suppression of the alpha band was found in the auditory cortex contralateral to the attended ear. The ipsilateral ear was largely characterized by enhancement of the alpha band, thought to reduce activation of that region as information presented to the unattended ear is meant to be ignored (Müller \& Weisz, 2012). This explanation for ERS in limited regions of the cortex is relatively common, and is often used to support the theory of gating-by-inhibition (Jensen \& Mazaheri, 2010).

Similar findings were revealed by Mazaheri et al. (2014). Using a cross-modal attention paradigm, participants were tasked to judge a visual orientation or an auditory pitch as was indicated by a preceding visual cue. Preparation for visual and auditory cues suppressed alpha power in the appropriate sensory cortex when a distractor was present alongside the target. Alpha power increased across the sensory region which was "ignored" on a trial-by-trials basis. The authors suggested, in concordance with additional evidence that the auditory alpha rhythm responds in the same manner as visual alpha and mu-alpha across the sensorimotor regions. More specifically, it appears that attention can modulate sensory cortex excitability (Mazaheri et al., 2014). However, these findings seem to only arise where studies include another sensory modality, and do not appears in studies featuring auditory-only stimulus, unless the stimuli are presented to individual ears. 
Using time-frequency analysis of ICA derived components from EEG data during speech discrimination and production tasks, Jenson et al. (2015) investigated alpha activity in the auditory cortex. Participants performed same/different discriminations on pairs of syllables included /ba/ and /da/ in both quiet and noisy backgrounds. Bilateral auditory alpha components were identified in only 15 of the 29 participants, suggesting that the auditory alpha component is more challenging to identify using EEG compared to the sensorimotor mu rhythm. In production conditions, auditory alpha power increased during production, consistent with speech-induced suppression, a phenomena thought to occur to reduce the impact of self-generated speech. The discrimination conditions were characterized by alpha ERS after stimulus offset. The ERS following stimulus offset was explained as indicative of the covert replay occurring in the anterior dorsal stream initiating a similar response to speech-induced suppression.

To the best of my knowledge, there is no current evidence establishing oscillatory sex-related differences in the auditory alpha rhythm. The best indicators of sex-related differences in the auditory system come from behavioral, neuroimaging, auditory evoked potentials, and event-related potential studies. To this end, Ruytjens et al. (2007) used positron emission tomography to investigation primary auditory cortical activation when listening to music, white noise, and nothing. Males (10) and females (10) experience greater auditory cortical activation to music compared to noise, but the difference was higher in men. Upon further investigation, it was found that this difference was driven by a higher activation in females compared to males in the noise condition. When comparing noise to the baseline condition, males showed relative deactivation of the prefrontal cortex, a finding not replicated in the females group. The authors suggest that sex-related differences in attention is likely to drive the prefrontal differences. This may, in turn, modulate auditory cortical activity, which could explain the sex differences. The authors indicate that sex is an important variable to consider when examining auditory processing in the brain.

Dehan and Jerger (1990) examined sex-related differences in the auditory brainstem response (ABR) which measures pre-attentive measures from the peripheral auditory nerve up to the inferior colliculus. Overall, males had lower amplitudes and higher latencies across the ABR. However, head size was additionally taken in to account, revealing that the differences in waves III and V latencies are somewhat explained by head size, as are some of the amplitude differences in wave V. Differences in monthly hormonal cycles in females also significantly altered the absolute latencies of wave $\mathrm{V}$. This suggested that these sex-related differences were linked to both head-size differences and hormone levels (Dehan \& Jerger, 1990). The ABR is unaffected by attention, thus these sex-differences are not the result of differences in general cognitive processes. As such, some basic sex-related differences appear to exist outside of general cognition.

Aerts and colleagues (2015) investigated the long latency responses for sexrelated differences in phoneme discrimination and word recognition tasks. Twenty men and twenty-four women participated in each task. The phoneme discrimination task 
consisted of two parts. There was a passive condition and an active condition, which measured the mismatch negativity (MMN) and P300 responses, respectively. Each condition used an oddball paradigm, though the instructions for the task of 'hitting a button upon hearing the oddball' was only used for the P300 acquisition. Standard and oddball only differed in one phonemic contrast. The word recognition task was a passive oddball tasks where pseudowords were the oddball and real words were used as the standard stimulus. Participants were asked to ignore the auditory stimuli and attend to the silent movie being presented. In the passive phoneme discrimination condition, MMN amplitude was significantly larger in women but only for phonemic contrasts with place of articulation. Latencies for the MMN were shorter in women over the right anterior portion of the head, while they were shorter for males over the left-central region of the head. Active discrimination, eliciting the P300, revealed larger amplitudes in females for the point of articulation contrasts. Word recognition revealed no sex-related differences in the N100 time window. Women were found to have shorter latency P200 responses compared to men in response to pseudowords. In the N400 time window, women were found to have longer latencies for pseudowords over real words compared to men. During the processing of real words, males had a longer latency than females in this time window. This study clearly indicates that sex-related differences can be found in both attentive and pre-attentive tasks that involve speech signals.

McFadden (1998) summarized a significant portion of physical and behavioral measures with sex-related differences. Physically, males have larger heads, pinnae, external ear canals, middle ear volumes, and longer cochleae. Psychophysically, females tend to have more sensitive hearing above $2 \mathrm{kHz}$, while in binaural tasks males are more sensitive to interaural time and intensity difference. Females have a smaller right ear advantage. Males tend to have more noise induced hearing loss while females have less temporary hearing loss below $1.5 \mathrm{kHz}$ and more above $3 \mathrm{kHz}$. In masking tasks, males are more sensitive to profile analysis and have more lateral suppression, females have more masking overshoot. Females tend to be more sensitive in gap detection tasks (McFadden, 1998). McFadden also noted that the hormonal cycle alters these responses and can make these differences smaller.

Much like behavioral differences, there are many differences found in males and females both cortically and psychophysically in auditory tasks which may or may not evoke different oscillatory effects in the auditory regions of the cortex. These difference do suggest, however, that the possibility is worth investigating. Specifically, sex-related differences found in studies with speech stimuli point to the presence of sex-related differences in the auditory cortex. 


\section{CHAPTER 3. METHODOLOGY}

\section{Participants}

To ensure cluster inclusion of at least $70-80 \%$ as found in previous studies employing these methods (Jenson et al., 2014; Nyström, 2008; Saltuklaroglu et al., 2017; Thornton et al., 2017), 59 English-speaking adults (28 males and 31 females) were recruited from the greater Knoxville area to participate in this study. The goal was to ensure at least 21 participants per group were included in final analyses. To minimize the effects of hormonal fluctuations on cortical activity, all female participants confirmed use of oral contraceptives via self-report (McFadden, 1998). The participants are age and handedness matched such that one male is matched to one female. Participants are between the ages of 18-30, and average ages of each group do not differ by $>1$ year, similar to the method employed by Upadhayay and Guragain (2014). Self-report was used to confirm no history of cognitive or communicative disorders in any participants. To confirm handedness, the Edinburgh Handedness Inventory (Edlin et al., 2015; Oldfield, 1971) was given to every participant. This study is approved by the Institutional Review Board of the University of Tennessee and each participant will sign an informed consent document approved by the review board. All data was collected in concordance with the Declaration of Helsinki.

\section{Stimuli}

Syllable stimuli /ba/ and /da/ were generated using NeoSpeech Software, which uses synthetic analogs of human male and female speakers to create utterances. Syllable stimuli were then exported from NeoSpeech and imported to Audacity to create syllable pairs. Within Audacity, /ba/ and /da/ syllables were combined in to 4 pairs spoken by the male and female speakers. Pairs were created such that 2 of the 4 consisted of the same syllable (e.g. /ba/ /ba/) and 2 consisted of different syllables (e.g. /da/ /ba/). Each syllable within each pair is $200 \mathrm{~ms}$ in duration, with a $200 \mathrm{~ms}$ silent block between offset of the first syllable, and onset of the second syllable, as shown in Figure 3-1. The control condition, featuring white noise, consisted of $3500 \mathrm{~ms}$ of white noise, with $2000 \mathrm{~ms}$ occurring before time point 0 (stimulus onset in the experimental conditions) and $1500 \mathrm{~ms}$ following time point 0 . Stimuli were normalized for root-mean-square (RMS) amplitude after low-pass filtering below $5 \mathrm{kHz}$. Syllables are used to as they are not linguistically loaded and should not significantly activate the auditory ventral stream (Cuellar, Bowers, Harkrider, Wilson, \& Saltuklaroglu, 2012; Kotz et al., 2010) and will allow for direct comparisons with previous studies (Bowers, Saltuklaroglu, Harkrider, \& Cuellar, 2013; Jenson et al., 2014; Jenson, Harkrider, Thornton, Bowers, \& Saltuklaroglu, 2015; Thornton et al., (in prep)). 


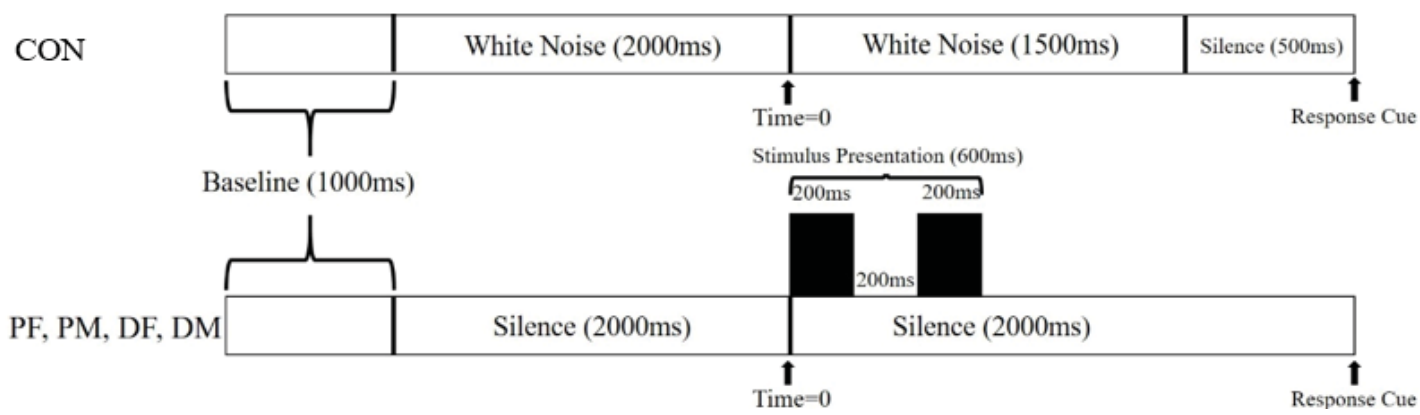

Figure 3-1. Stimulus Presentation Timeline. 


\section{Design}

Five conditions were used in a within and between group design:

- Passive listening to white noise (CON), the control condition

- Passive listening to syllables produced by a synthetic female speaker (PF)

- Passive listening to syllables produced by a synthetic male speaker (PM)

- Discrimination (same or different) of syllables within a pair spoken by a synthetic female speaker (DF)

- Discrimination (same or different) of syllables within a pair spoken by a synthetic male speaker $(\mathrm{DM})$

\section{Procedure}

Participants were seated in a comfortable recliner in a double walled, sound and electromagnetically shielded booth. Stimuli were presented via the Stim 2 4.3.3 software through insert earphones (ER-14A) at $75 \mathrm{~dB}$. Each condition consisted of 80 trials presented over 2 blocks. Conditions with speech stimuli contained 40 pairs with the 'same' syllables, and 40 with 'different' syllables. Within each condition, pairs were presented randomly. Condition presentation were pseudorandomized, such that passive listening to speech conditions were presented before the active discrimination conditions to avoid the possible influence of neural activity related to discrimination being carried into passive listening tasks. Each condition required the participant to press a button to move on to the next trial. This task was included to ensure the participants remained awake and alert while completing each condition. The cue to respond was a $100 \mathrm{~ms}, 1$ $\mathrm{kHz}$ 'chirp' that followed stimulus offset by $1400 \mathrm{~ms}$ (500ms in CON condition). In discrimination conditions, pushing the left button with the left hand indicated the listener heard a 'same' pair, while pushing the right button with the right hand indicated a 'different' pair. The even distribution of 'same' and 'different' trials was used to compensate for response bias (Venezia, Saberi, Chubb, \& Hickok, 2012). Further, including a motor response is known to suppress the mu rhythm (Graimann \& Pfurtscheller, 2006; Hari, 2006). To compensate for this, the passive conditions also included the motor response and balance between left and right responses, to ensure consistency. Thus, any differences late in the trial are not due to the response requirement. Behavioral performance for the active discrimination conditions were calculated as a percentage of a correct trials.

\section{EEG Acquisition}

EEG data was collected using a 64 channel unlinked, sintered Neuroscan EEG Quik cap with electrodes configured to the international extended 10-20 array with additional electrodes placed above, below, and each side of the left eye, above and below the lips, and on the mastoids. The mastoid electrodesserved as a linked reference for all recording electrodes. Compumedics NeuroScan 4.3.3 software and the Synamps 2 system 
were used to collect the behavioral and EEG data during the experiment, with stimuli being presented via the Stim2 software which marks raw EEG data with stimulus onset markers. Raw files are band-pass filtered from .1-100Hz and digitized by a 24-bit analog to digital converter with a sampling rate of $500 \mathrm{~Hz}$. These continuous EEG data files contained event markers representing stimulus onset and participant responses.

\section{EEG Data Processing}

EEGLAB 2014b, an open source MATLAB toolbox was used to process EEG data. Both individual and group processing steps will be utilized and are detailed below:

- Individual Processing

- Raw EEG data from each condition for each participant was processed via the pipeline described below.

- Independent component analysis (ICA) was performed on concatenated data within each participant (CON, PM, PF, DM, DF).

- Dipole localization for each independent component (IC) as revealed by ICA was then calculated.

- Group Analysis

- Principal component analysis (PCA) was used to cluster components from each participant based on pre-defined inclusion criteria using the EEGLAB STUDY module.

- Right/left mu and right/left auditory component clusters were clustered by PCA using spectra, scalp maps, and dipole locations as inclusion criteria.

- Event-related spectral perturbations (ERSPs) were used to examine changes in the oscillatory activity before, during, and after stimulus onset in both mu and auditory clusters.

Further details regarding individual and group processing of EEG data can be found in the following section.

\section{Individual Processing/Analysis of EEG Data}

Data from each condition underwent a series of processing steps before ICA. Data were: 1) downsampled to $256 \mathrm{~Hz}$ to reduce computational load during later processing steps; 2) filtered from 3-50Hz to reduce slow drift in EEG data and reduce overall data load for further computational steps; 3 ) epoched around stimulus onset at time point zero, with 3 seconds before stimulus onset and 2 seconds after. This creates 5 second epochs from -3 to 2 around stimulus onset; 4) re-referenced to linked mastoid electrodes; 5) visually inspected for gross artifact $(>200 \mathrm{uV})$ with bad channels removed; and 6) incorrect or late response trials were removed from EEG data. Responses were considered 'late' if the response occurs more than 2 seconds following the response cue. For later analyses to be successful, at least 40 clean trials in each condition were required 
from each participant. The overall average, based on previous studies, was expected to comfortably exceed this 40 trial cutoff.

\section{Independent Component Analysis (ICA) of EEG Data}

For each participant, ICA weights was calculated using the preprocessed EEG data from each of the five conditions. This method enables accurate comparison of activity across conditions within revealed ICs, as each component is reflecting activity within a region of the cortex found in each condition. ICA training was performed using the "extended runica" algorithm in EEGLAB and will be set to an initial learning rate of 0.001 with a stopping weight of $10^{-7}$. Each participant returned up to 64 ICs (minus removed channels) following decomposition, which reflects the number of recording channels and excludes the reference channels (the mastoid electrodes). To obtain scalp maps for each IC from each participant, the inverse weight matrix (W-1) was projected on to the spatial EEG channel configuration.

\section{Dipole Fitting of EEG Data}

Following ICA decomposition, equivalent current dipole (ECD) models were computed for each component. Using the DIPFIT toolbox, an extension of the EEGLAB toolbox (Oostenveld \& Oostendorp, 2002), dipoles are fit to the ICA decomposition using the brain electrical source analysis (BESA) model. Dipole processing will produce a single dipole for every component from each participant. Localization of generated dipoles to a cortical source relies on back projection to a signal source which likely generated the scalp potential distribution for each individual IC. After back projection, the best forward model is computed based with the goal of explaining the most scalp variance (Delorme, Palmer, Onton, Oostenveld, \& Makeig, 2012). Any remaining unexplained variance, which is known as residual variance (RV), can be considered a measure of 'goodness of fit' between the scalp map and the generated ECD model. In other words, lower RV is indicative of a less unexplained variability, and thus a better fit.

\section{Group EEG Data Analysis}

Group data analysis was performed using the EEGLAB STUDY module. This module allows ICA/Dipole data from all participants to be examined between experimental conditions. At this step, dipoles with an RV of greater than $20 \%$ were excluded from analysis. This step is taken to increase confidence in localization and reduces computational load. 


\section{Principal Component Analysis (PCA) of EEG Data}

PCA was used to cluster components from all participants based on dipole location, component spectral characteristics, and scalp topography. The K-means toolbox was employed using the previously mentioned criteria to yield clusters of components. A cluster in the left and right hemispheres is expected to be characterized by mu rhythm spectra with dipole localization around Brodmann's Areas (BA) 2, 3, 4, or 6. Auditory clustersinclude components characterized by alpha dominated spectra and dipoles localized to BA 21, 22, 41, or 42. Additional visual inspection was performed on nearby component clusters to identify any inappropriately clustered components from any participants. The left/right mu and left/right auditory clusters were then internally matched in order to ensure even male and female contribution.

\section{Time-Frequency Analysis of EEG Data}

To investigate changes in mu-alpha/mu-beta and auditory alpha spectral power relative to the tasks, event-related spectral perturbations (ERSPs) was employed. A Morlet sinusoidal wavelet set to 3 cycles at $3 \mathrm{~Hz}$ and 34 cycles and $34 \mathrm{~Hz}$ with a linear rise will be used to extract time-frequency transforms. Each trial was referenced to its own $1000 \mathrm{~ms}$ baseline which was selected from the inter-trial interval. Using 200 randomly selected latencies from within the inter-trial interval, a surrogate distribution was constructed. From this distribution, changes from the baseline in ERSPs were computed using a bootstrap resampling method ( $p<0.05$ uncorrected).

Inter-condition differences were assessed using Montecarlo statistics. Significant difference thresholds will be set at $p<0.05$, with type 1 error rates controlled for with the cluster correction (CC) method (Pernet, Latinus, Nichols, \& Rousselet, 2015). Two 2x5 repeated measures ANOVAs (Males and females, CON, PM, PF, DM, and DF conditions) were performed to analyze between group differences and to determine whether within group differences exist relative to the control $(\mathrm{CON})$ condition. Additional statistical comparisons examined within and between group differences and compare patterns of neural activity in smaller data subsets (e.g. PM vs DM within each group). 


\section{CHAPTER 4. RESULTS}

\section{Behavioral Results}

Consistent with previous studies (Bowers et al., 2013; Jenson et al., 2014; Saltuklaroglu et al., 2017; Thornton et al., 2017), accuracy was high in both the DF and DM conditions, with every participant achieving $>90 \%$. Thus, no significant differences were noted between groups $(\mathrm{F}(1,54)=0.152, p=0.698)$ nor between conditions $(\mathrm{F}(1,54)=0.563, p=0.456)$. No significant interaction (Sex*Accuracy) was found $(\mathrm{F}(1,54)=1.849, p=0.18)$. Inaccurate trials were removed from further analysis to avoid influence of difference cortical processes that may be related to incorrect responses.

Response times (RT) were recorded to determine average response times between conditions and groups, while also confirming active participation from each listener. No significant differences were noted between groups $(\mathrm{F}(1,54)=1.966, p<0.167)$ and no condition differences were found $(\mathrm{F}(4,54)=1.178, p=0.321)$. Additionally, no significant interaction $(\operatorname{Sex} * \mathrm{RT})$ was revealed $(\mathrm{F}(4,54)=0.484, p=0.596)$. Any trial which exceeded a participant's mean RT by $>2$ standard deviations was excluded from further analysis.

\section{Neurophysiological Results}

Due to data collection failure, one male and two female datasets did not yield usable data. Thus, analysis was performed on 27 males and 29 females. EEG data were cleaned of any trials with significant artifact, ensuring that ICA was performed on a clean recording for each participant. Mean usable trials for each condition was 61.96 (CON), 64.89 (PF), 63.96 (PM), 64.27 (DF), and 62.75 (DM).

\section{Mu Cluster Characteristics}

Mu components were identified via ICA in both the left and right hemisphere. Overall mu cluster contributions were consistent with previous studies (Bowers et al., 2013; Jenson et al., 2014; Thornton et al., 2017), with 20 males ( 74\%) and 22 females $(\sim 75 \%)$ contributing to the left mu cluster, and 22 males $(\sim 81 \%)$ and 26 females $(\sim 90 \%)$ contributing to the right mu cluster. Thus, males contributed 33 components to the left mu cluster and 30 to the right mu cluster, while females contributed 30 components and 28 components to the left and right mu clusters, respectively. Information regarding source localization of left and right mu clusters can be found in Table 4-1.

\section{Auditory Cluster Characteristics}

ICA successfully identified auditory components in both the left and right hemisphere. Overall auditory cluster contributions were marginally consistent with a 
Table 4-1. Source Localization Information for Mu Clusters.

\begin{tabular}{lll}
\hline Source Information & Left Mu & Right Mu \\
\hline By Group & & \\
Males: Mean ECD source & {$[-38,-15,32]$ (BA 6) } & {$[38,-9,36]$ (BA 6) } \\
Mean RV & $472 \%$ & $4.84 \%$ \\
Females: Mean ECD source & {$[-38,-14,39]$ (BA 4) } & {$[40,-8,40]$ (BA 3) } \\
Mean RV & $3.92 \%$ & $3.57 \%$ \\
Distance between sources & $7 \mathrm{~mm}$ & $4.5 \mathrm{~mm}$ \\
& & \\
Male and Females groups combined & {$[-38,-15,35]$ (BA 4) } & {$[39,-8,38]$ (BA 6) } \\
Mean ECD source & $4.34 \%$ & $4.23 \%$ \\
Mean RV & & \\
\hline
\end{tabular}


previous study that examined auditory activity in speech perception using ICA derived components (Jenson et al., 2015a), with 17 males $(\sim 62 \%)$ and 17 females $(\sim 58 \%)$ contributing to the left auditory cluster, and 11 males $(\sim 42 \%)$ and $12(\sim 41 \%)$ females contributing to the right auditory cluster. It was possible for participants to contribute multiple components to each cluster. Thus, males contributed 19 components to the left auditory cluster, and 14 to the right auditory cluster, while females contributed 21 components and 18 components to the left and right auditory clusters, respectively. Table 4-2 provides information regarding source localization of left and right auditory clusters.

\section{Does Speaker Sex Influence Anterior Dorsal Stream Processing?}

The first hypothesis suggested that anterior dorsal stream activity will be characterized by increased oscillatory alpha power when listening to speakers of the opposite sex, aligning with supporting evidence from previous studies (Gootjes et al., 2006; Thornton, Harkrider, Jenson, \& Saltuklaroglu, 2019). Assessing anterior dorsal stream activity, Figure 4-1 and Figure 4-2 reveal that patterns of alpha ERS are not consistently present in either the female or male group regardless of speaker sex. As such, no significant differences are noted between groups when statistical corrections (Cluster Correction) are applied. Removal of these corrections, as shown in the figures, revealed scattered patterns of differences between females and males. Only the DF condition in the right mu cluster slightly supported the hypothesis, showing the males were characterized by greater mu alpha ERS around stimulus onset.

\section{Does Increasing Task Demands Increase Dorsal Stream Activity?}

Increasing task demands $(\mathrm{PF}+\mathrm{PM}$ vs $\mathrm{DF}+\mathrm{DM})$ by introducing the discrimination task revealed significant differences within both the female and male groups, to varying degrees both the mu and auditory clusters in both hemispheres. Both females and males revealed stronger beta ERD after stimulus offset in the left hemisphere mu cluster, while females additionally were characterized by stronger alpha ERD during stimulus presentation and after stimulus offset (Figure 4-3). The right hemisphere mu cluster only revealed differences in the female group relative to task demands. In the active discrimination conditions, females continued to display stronger alpha and beta ERD during and after stimulus presentation in the right mu cluster (Figure 4-4).

The auditory alpha clusters also revealed differential activity based on task demands within the female and male groups. Females displayed stronger alpha ERD in the left auditory alpha cluster in the active discrimination conditions, occurring during stimulus presentation, while no differences were found in the right auditory cluster (Figure 4-5). Males, interestingly, were characterized by stronger beta ERD during stimulus presentation in the active discrimination conditions in the left hemisphere auditory alpha. In addition, males were characterized by stronger alpha ERS after stimulus offset in the right hemisphere auditory alpha cluster in the active discrimination conditions (Figure 4-6). 
Table 4-2. Source Localization of Auditory Clusters.

\begin{tabular}{lll}
\hline Source Information & Left Auditory & Right Auditory \\
\hline By Group & & \\
Males: Mean ECD source & {$[-58,-47,-1]$ (BA 21) } & {$[56,-36,12]$ (BA 42) } \\
Mean RV & $7.15 \%$ & $6.30 \%$ \\
Females: Mean ECD source & {$[-53,-47,0]$ (BA 22) } & {$[57,-41,2]$ (BA 22) } \\
Mean RV & $5.45 \%$ & $7.68 \%$ \\
Distance between sources & $5 \mathrm{~mm}$ & $11.2 \mathrm{~mm}$ \\
& & \\
Male and Females groups combined & {$[-56,-47,0]$ (BA 21) } & {$[57,-39,6]$ (BA 22) } \\
Mean ECD source & $6.38 \%$ & $7.08 \%$ \\
Mean RV & & \\
\hline
\end{tabular}



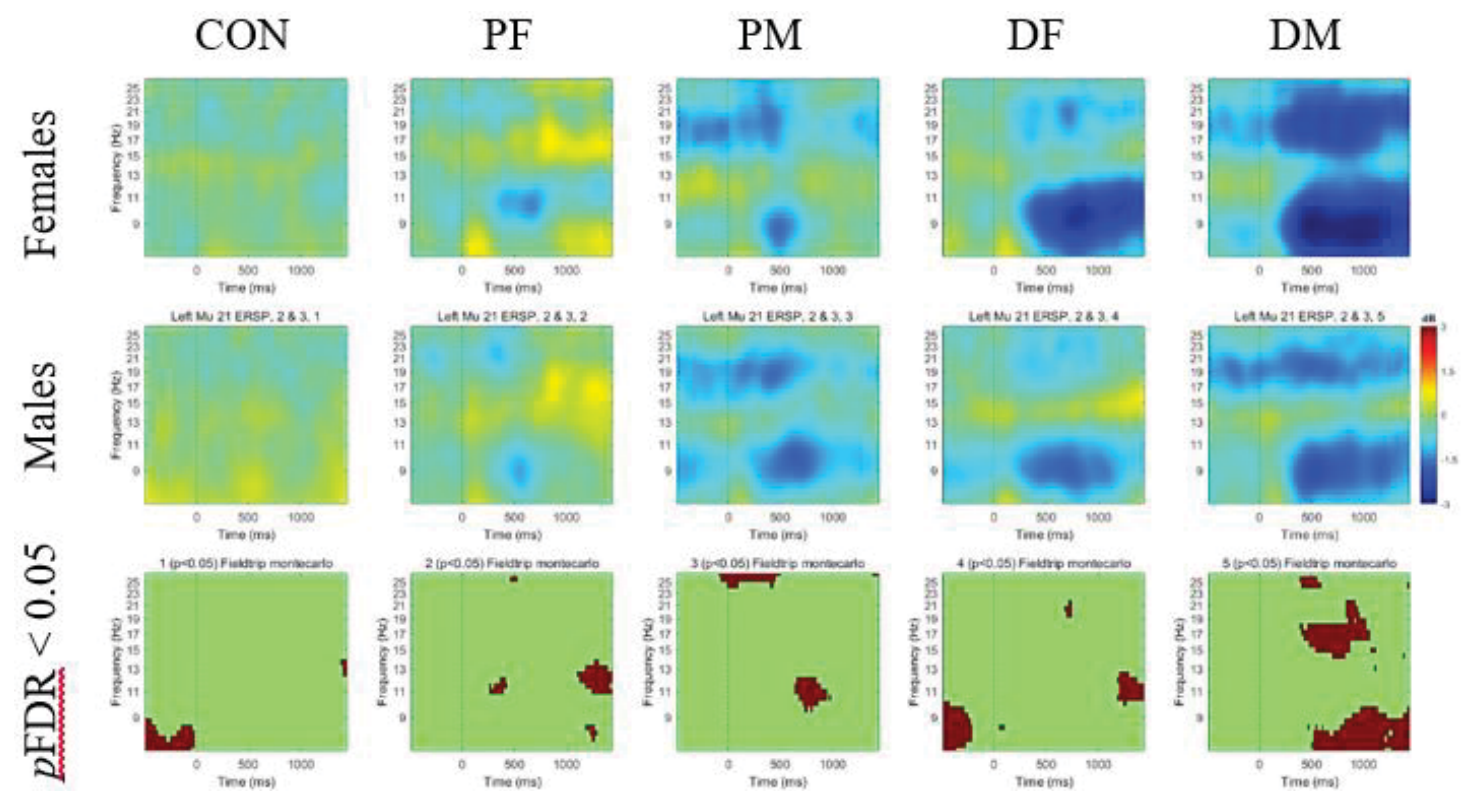

Figure 4-1. Left Hemisphere Mu Cluster Time-Frequency Analysis.

Mean ERSPs across all test conditions (warm colors indicate ERS whereas cool colors indicate ERD). For each condition, ERSPs display changes from a recorded baseline before stimulus onset ( -3000 to $-2000 \mathrm{~ms}$ before stimulus onset for each trial). The final panel in each column indicates statistical differences between each group within test conditions $(p \mathrm{FDR}<0.05$, uncorrected). 

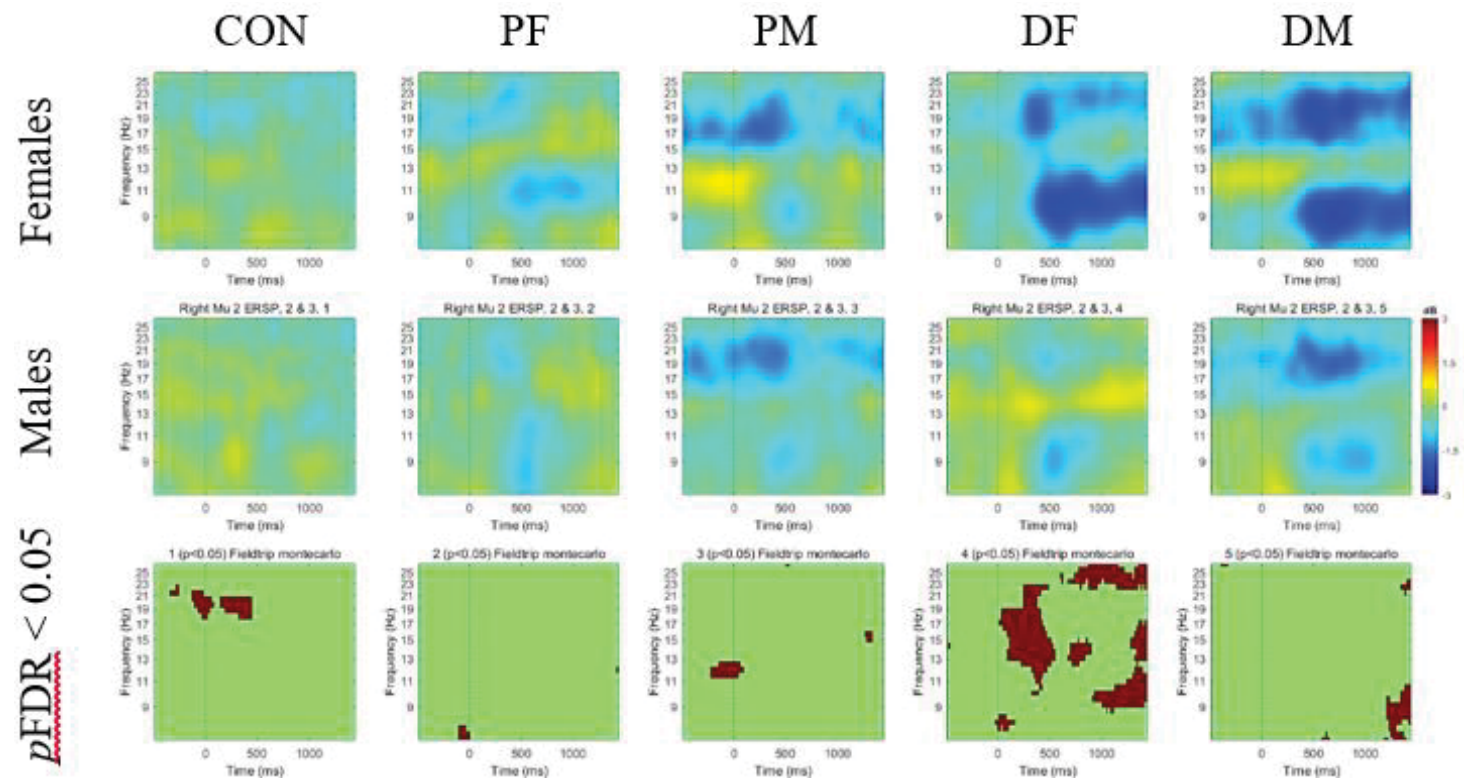

Figure 4-2. Right Hemisphere Mu Cluster Time-Frequency Analysis.

Mean ERSPs across all test conditions (warm colors indicate ERS whereas cool colors indicate ERD). For each condition, ERSPs display changes from a recorded baseline before stimulus onset (-3000 to $-2000 \mathrm{~ms}$ before stimulus onset for each trial). The final panel in each column indicates statistical differences between each group within test conditions ( $p \mathrm{FDR}<0.05$, uncorrected). 

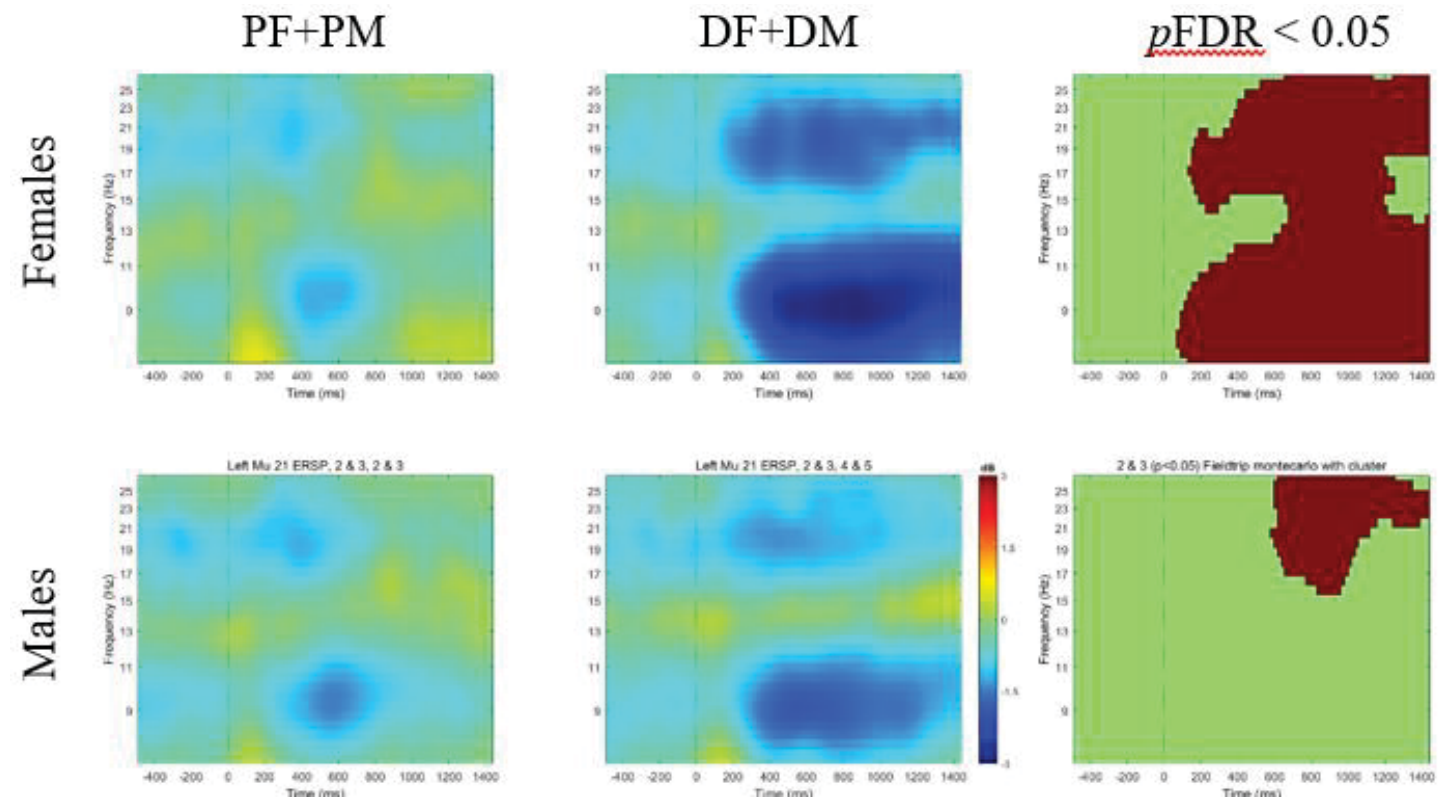

Figure 4-3. Left Hemisphere Mu Cluster Time-Frequency Analysis Task Comparison.

Mean ERSPs across test conditions grouped by task (passive vs active, warm colors indicate ERS whereas cool colors indicate ERD). For each condition, ERSPs display changes from a recorded baseline before stimulus onset ( -3000 to $-2000 \mathrm{~ms}$ before stimulus onset for each trial). The final panel in each column indicates statistical differences within each group across test conditions ( $p$ FDR $<0.05$, corrected). 

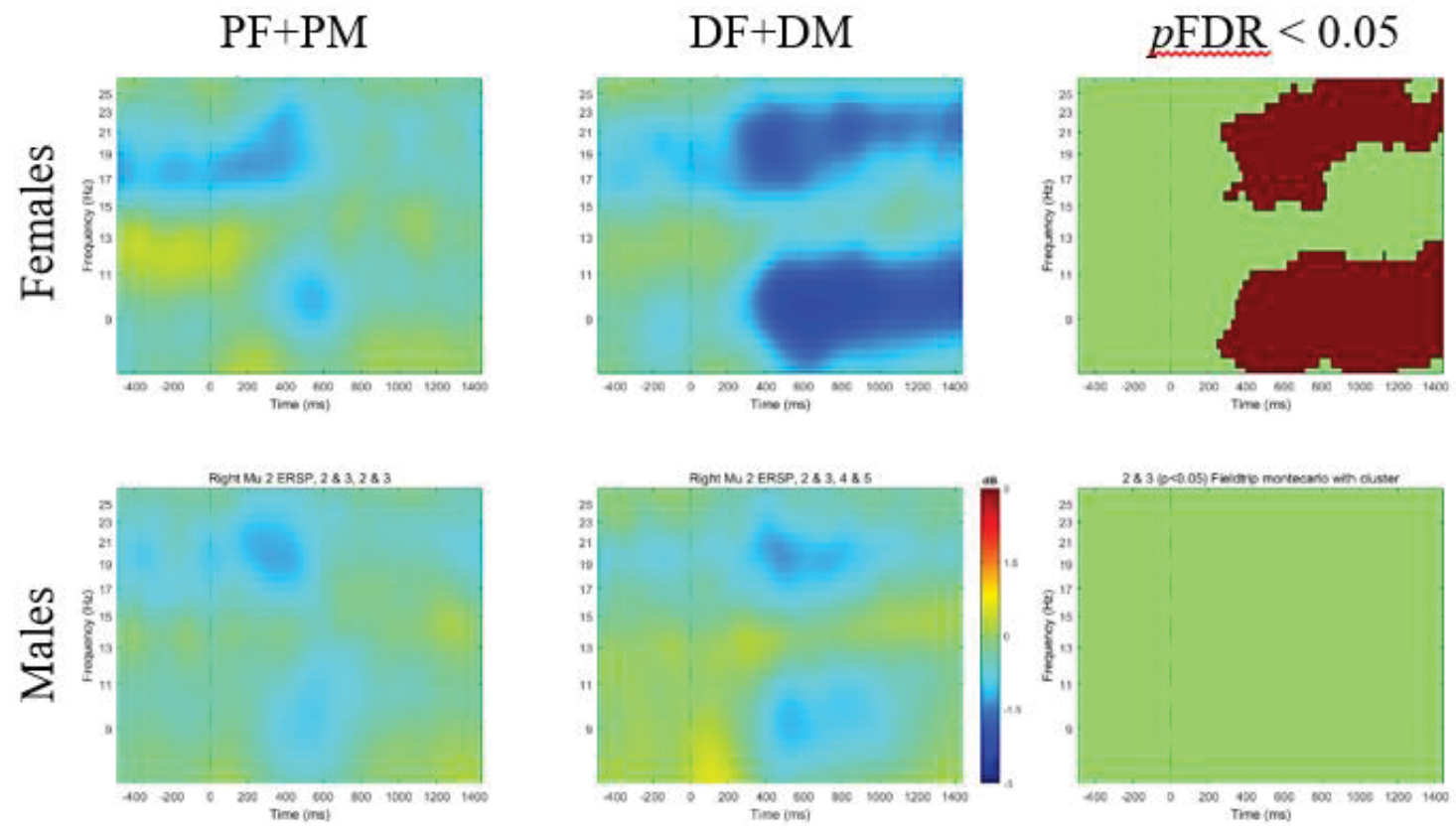

Figure 4-4. Right Hemisphere Mu Cluster Time-Frequency Analysis Task Comparison.

Mean ERSPs across test conditions grouped by task (passive vs active, warm colors indicate ERS whereas cool colors indicate ERD). For each condition, ERSPs display changes from a recorded baseline before stimulus onset ( -3000 to $-2000 \mathrm{~ms}$ before stimulus onset for each trial). The final panel in each column indicates statistical differences within each group across test conditions ( $p$ FDR $<0.05$, corrected). 

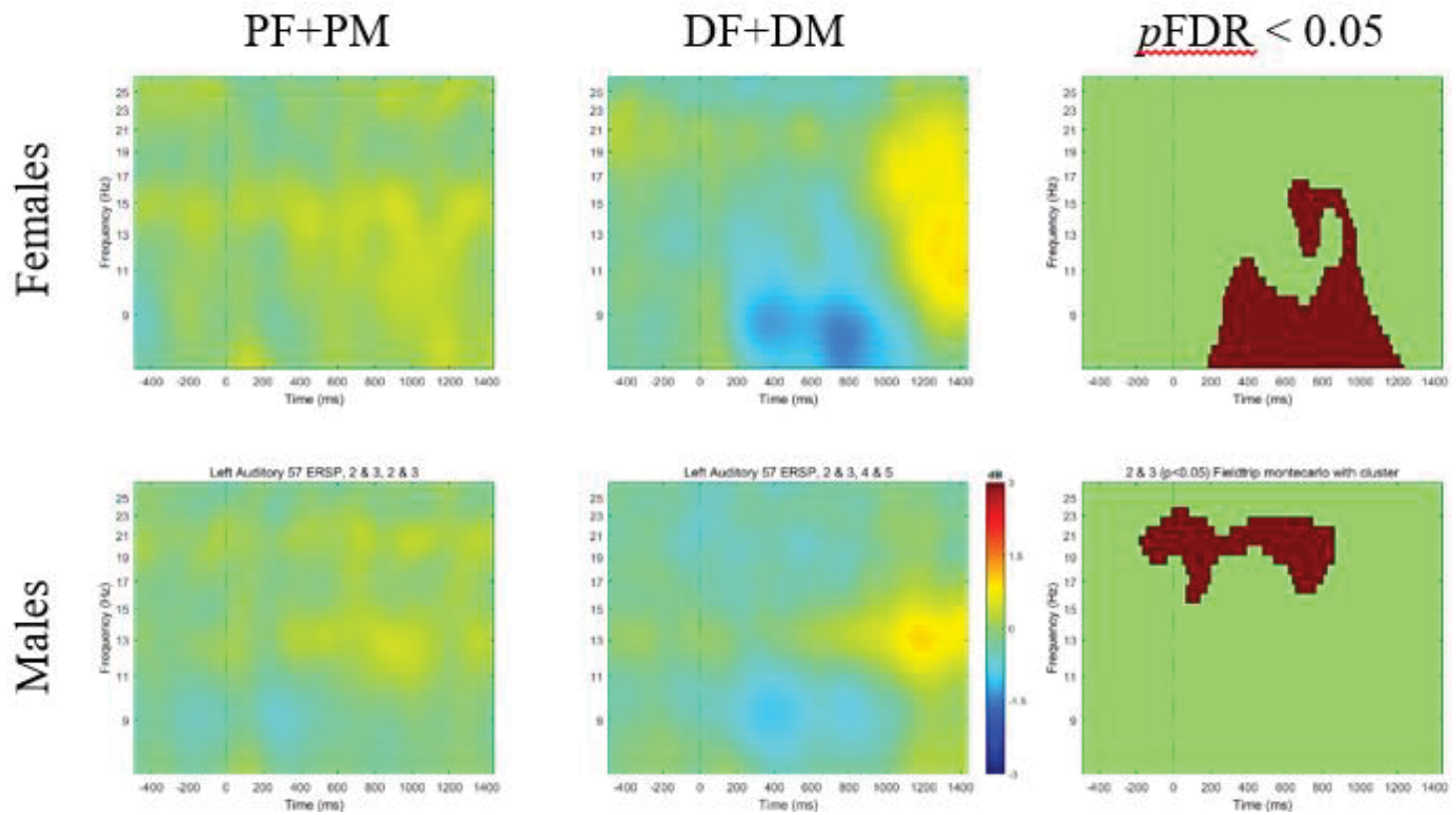

Figure 4-5. Left Hemisphere Auditory Cluster Time-Frequency Analysis Task Comparison.

Mean ERSPs across test conditions grouped by task (passive vs active, warm colors indicate ERS whereas cool colors indicate ERD). For each condition, ERSPs display changes from a recorded baseline before stimulus onset $(-3000$ to $-2000 \mathrm{~ms}$ before stimulus onset for each trial). The final panel in each column indicates statistical differences within each group across test conditions ( $p$ FDR $<0.05$, corrected). 

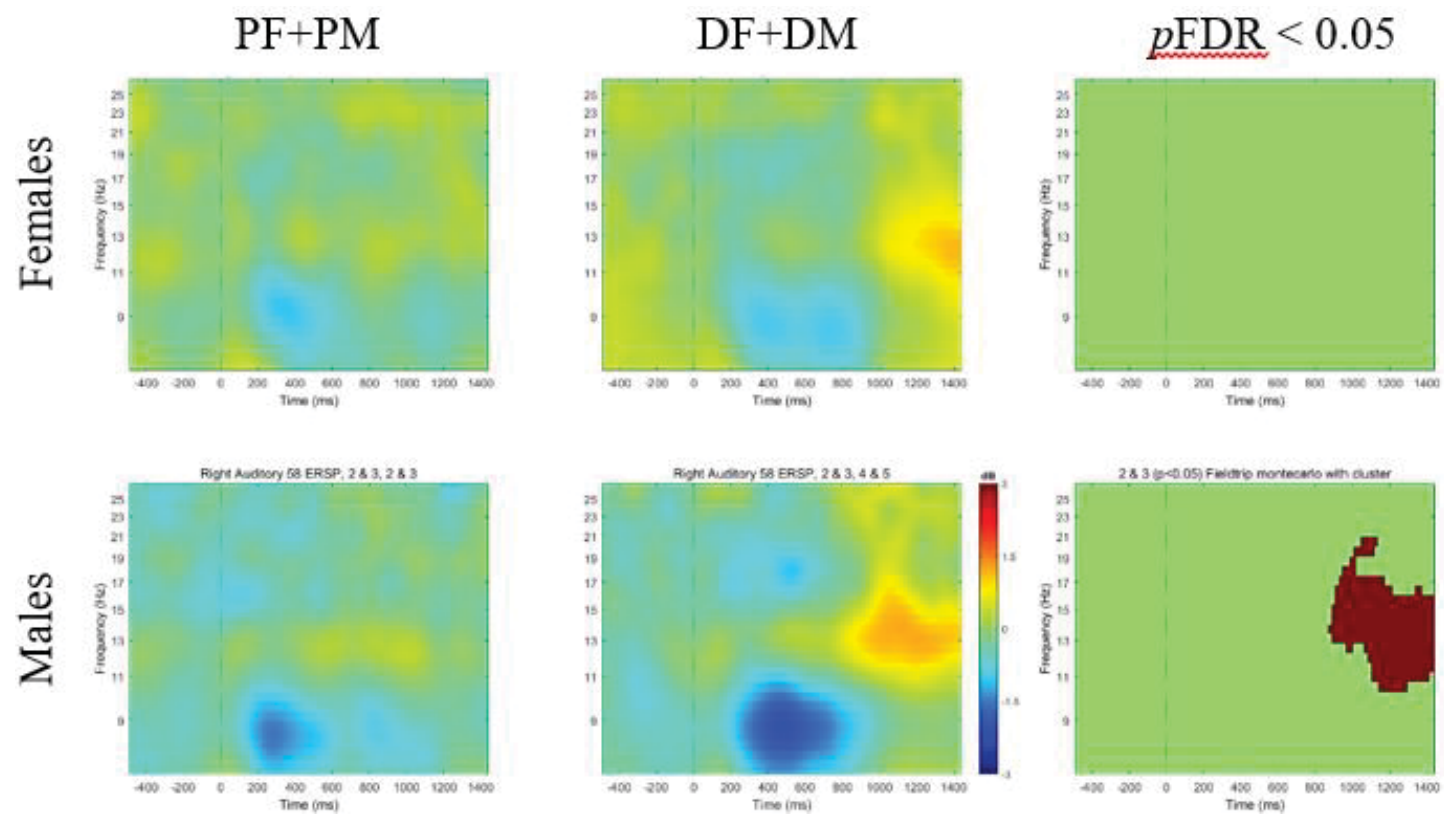

Figure 4-6. Right Hemisphere Auditory Cluster Time-Frequency Analysis Task Comparison.

Mean ERSPs across test conditions grouped by task (passive vs active, warm colors indicate ERS whereas cool colors indicate ERD). For each condition, ERSPs display changes from a recorded baseline before stimulus onset (-3000 to $-2000 \mathrm{~ms}$ before stimulus onset for each trial). The final panel in each column indicates statistical differences within each group across test conditions ( $p$ FDR $<0.05$, corrected). 


\section{The Effects of Speaker Sex Within Group}

While not specifically addressed by either hypotheses, post-hoc analysis within each group revealed surprising patterns of anterior dorsal stream activity when comparing the effect of speaker sex across task demand (i.e. PF+DF vs. PM+DM). Figure 4-7 and Figure 4-8 reveal that both males and females are characterized by stronger beta ERD before, during, and after stimulus presentation when listening to a male speaker compared to a female speaker. This pattern is consistent in both the left and right hemisphere mu component clusters, but is not reflected in the auditory clusters. 

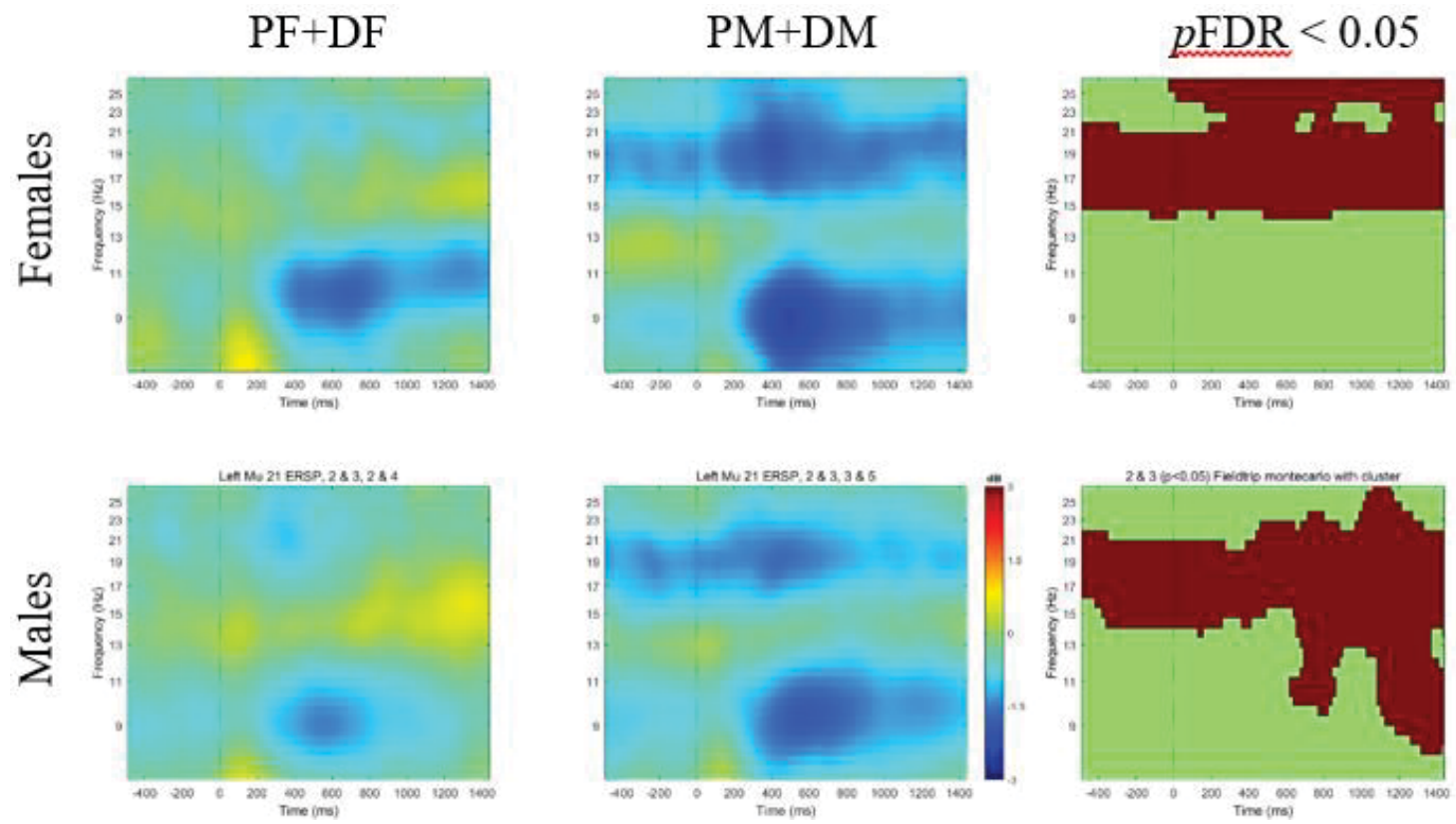

Figure 4-7. Left Hemisphere Mu Cluster Time-Frequency Analysis Speaker Comparison.

Mean ERSPs across test conditions grouped by speaker sex (female speaker vs male speaker, warm colors indicate ERS whereas cool colors indicate ERD). For each condition, ERSPs display changes from a recorded baseline before stimulus onset $(-3000$ to $-2000 \mathrm{~ms}$ before stimulus onset for each trial). The final panel in each column indicates statistical differences within each group across test conditions $(p \mathrm{FDR}<0.05$, uncorrected). 

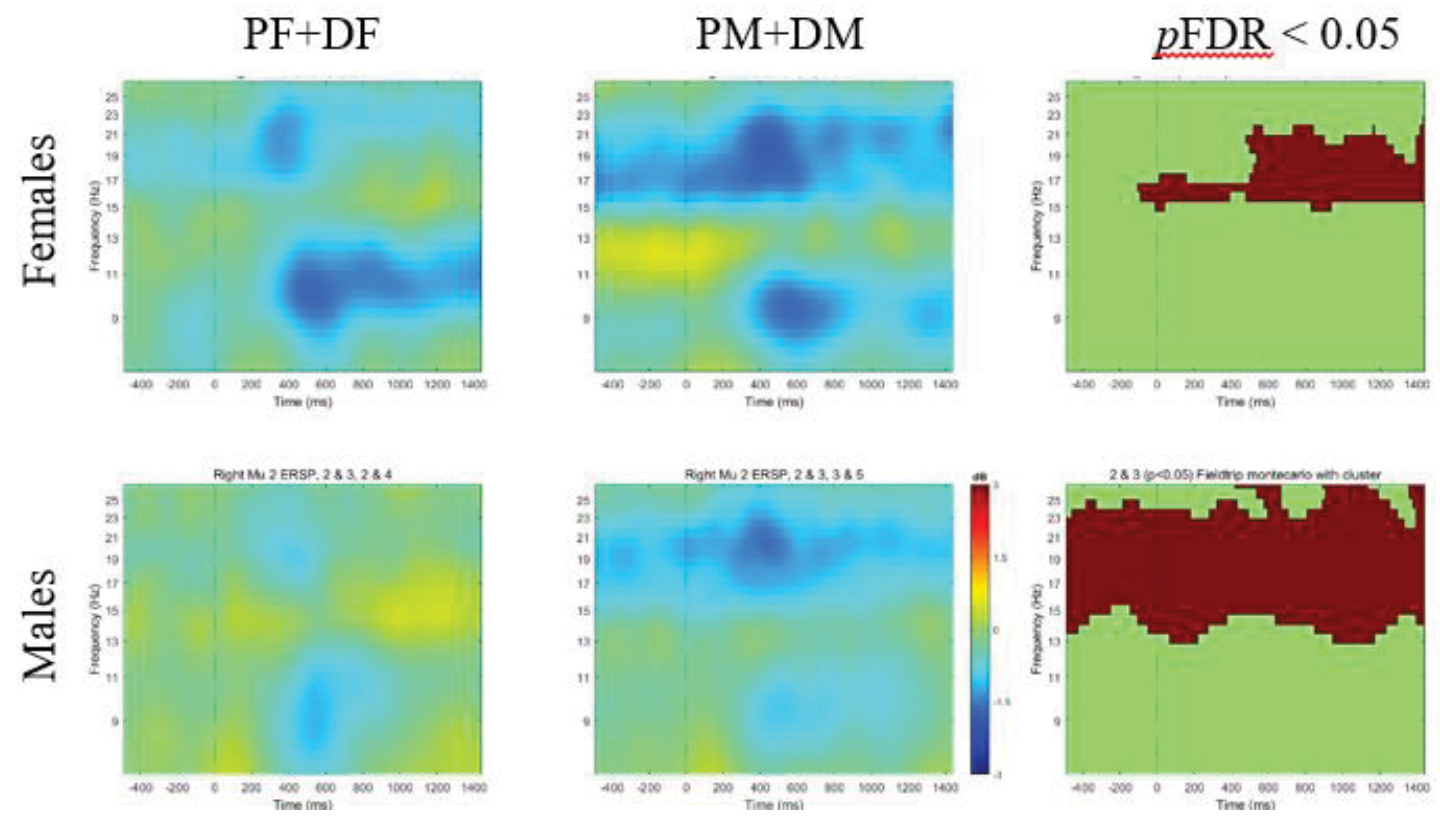

Figure 4-8. Right Hemisphere Mu Cluster Time-Frequency Analysis Speaker Comparison.

Mean ERSPs across test conditions grouped by speaker sex (female speaker vs male speaker, warm colors indicate ERS whereas cool colors indicate ERD). For each condition, ERSPs display changes from a recorded baseline before stimulus onset (-3000 to $-2000 \mathrm{~ms}$ before stimulus onset for each trial). The final panel in each column indicates statistical differences within each group across test conditions $(p \mathrm{FDR}<0.05$, uncorrected). 


\section{CHAPTER 5. DISCUSSION}

The goal of this study was to use time-frequency analysis of both EEG mu and auditory alpha rhythms to investigate effects of both speaker and listener sex on cortical processing during both passive listening and active speech discrimination tasks. Both females and males completed active discrimination conditions with high behavioral accuracy. In addition, males and females contributed similar numbers of ICA derived components to the left and right mu and auditory alpha clusters. Mu rhythm activity was localized to BA 6/4 for each mu cluster (left/right hemispheres), and BA 21/22 for the auditory alpha clusters. These sources of activity are consistent with previous studies examining mu and auditory activity (Jenson et al., 2014, 2015; Thornton et al., 2017). Together, these regions are consistent with regions of the auditory dorsal stream identified with fMRI (Burton et al., 2000; Szenkovits, Peelle, Norris, \& Davis, 2012). Further investigation of possible differences in cortical processing of passive and active speech discrimination tasks based on sex of the listener and speaker are supported by the similarities between males and females in performance of the tasks, spectral shape, and successful source localization. Consistent with Thornton et al., (2019), the results suggest that time-frequency analysis will describe differential implementation of cortical resources based on the sex of the listener and the sex of the speaker.

\section{Behavioral Data}

No performance differences were noted across conditions within or between sexes regarding both accuracy and response time. This is in conflict with other studies which have revealed sex differences in reaction times as well as accuracy in some situations. Generally, females tend to outperform males on a variety of language tasks, while males perform better in spatial processing tasks (Li, 2014). Reaction times, however, have conflicting and complicated results across other studies. While some studies reveal no differences (Georgiev, Minchev, Christova, \& Philipova, 2011), others have shown that males have generally faster reaction times to auditory and verbal stimuli (Jain, Bansal, Kumar, \& Singh, 2015). Further still, some studies measuring reaction time have shown effects linked to listener and speaker sex (Junger et al., 2013; Smith et al., 2018). The behavioral results from this study suggest either that sex differences in accuracy and reaction time are not present, or that the stimuli used were not sufficiently complex to elicit sex-related differences. This seems likely given the high overall accuracy across conditions in which a decision was required.

\section{The Influence of Speaker Sex on Anterior Dorsal Stream Processing}

Several fMRI studies have suggested that males and females recruit different regions of the brain to process speech (for review see Ruigrok et al., 2013). Emerging evidence also indicates that males and females process speech and non-speech stimuli differently within the same regions of the brain (Junger et al., 2013; Li et al., 2014; 
Popovich et al., 2010; Proverbio, Riva, Martin, \& Zani, 2010; Smith et al., 2018). The uncorrected results from this study suggest that males and females may differ in processing of speech signals, and these differences may depend on the sex of the speaker.

Figure 4-1 and Figure 4-2 display uncorrected results for the left and right mu clusters, respectively. Revealed differences are minor and diffuse in both the left and right hemispheres. Discernable patterns in the left hemisphere revealed that females display greater ERD in both the alpha and beta bands during and after stimulus onset in the active discrimination of male speakers, while in passive listening to a male speaker showed greater alpha ERD in males. Females appear to also be characterized by greater alpha ERD in the DF condition, as well as the PF condition, but these differences are more diffuse and less visually apparent. The greater ERD present in females in the DM condition is consistent with findings suggesting the presence of an opposite sex effect (Junger et al., 2013; Smith et al., 2018), possible based in biological relevance or mate selection. More resources are being allocated to processing the opposite sex speaker for this reason.

The right hemisphere mu cluster revealed fewer arising differences between males and females, but was characterized by greater ERS in females in the PM condition, and greater ERS in the DF condition in males. The stronger ERS in females during passive listening to male speakers doesn't necessarily align with an opposite sex effect, as ERS is associated with a reduction in processing. These findings are, however, more closely aligned with findings from Thornton et al., (2019) which found greater alpha ERS in females when actively discriminating a male speaker. Thornton et al., (2019) presented their findings as indicative of differential attention mechanisms being recruited and implemented by males and females to accomplish the same task. This study, however, attempted to remove the attentional demands by introducing passive listening tasks that still included speech signals. Greater alpha ERS in the females in the PM condition may reflect reduced mu reactivity and resonance when listening to a male speaker, indicating a reduction in automatic sensorimotor recruitment by opposite sex speakers. If this is region is accessed through sensorimotor processing demands such as auditory-to-motor mapping, it may be that females have reduced access to articulatory features when listening to a male speaker. This is also reflected in the male listeners when discriminating a female speaker. Higher alpha ERS appears to emerge in the same timeframe, early in stimulus presentation. Listening to opposite sex speakers may reduce recruitment of the anterior dorsal stream, at least in the alpha band. In the DF condition, females appear to be characterized by stronger post-stimulus alpha and beta ERD. Late activity is often associated with working memory, generally covert rehearsal, for maintaining the stimulus for the purpose of decision selection based on the task (Baddeley, 2012; Martha W. Burton \& Small, 2006; Jenson et al., 2014; LoCasto et al., 2004; Saltuklaroglu et al., 2017; Thornton et al., 2017). Reduced ERD in the right hemisphere in males may indicate reduced working memory recruitment in the DF condition, or possibly a shift to the left hemisphere, as no differences were noted in the left hemisphere between males and females. 
Across all conditions, no differences were noted in the mu cluster spectra. Consistent with Thornton et al., (2019), these results suggest that males and females have the same processing capacity for sensorimotor information in speech perception and discrimination tasks. While the ERSP patterns are weak and do not survive statistical correction, there is the possibility that these results are simply underpowered. More participants may strengthen these differences. Mu rhythm responses appear to be highly variable within populations, as such, between group differences are likely to require a significant group size.

\section{The Influence of Task Demands}

The effects of task demands was investigated by combining the passive conditions $(\mathrm{PF}+\mathrm{PM})$ and comparing mu and auditory activity to combined active discrimination conditions $(\mathrm{DF}+\mathrm{DM})$. This contrast did not explore any effects related to the sex of the listener, nor the sex of the speaker, instead examining mu and auditory reactivity as task demands were altered within each group (Figures 4-3 and 4-4). No spectral differences were found in either hemisphere for both the mu and auditory clusters.

\section{Mu Rhythm}

Significant differences were found between the passive listening and active discrimination conditions within the female and male groups. Females displayed greater alpha and beta ERD in the active discrimination conditions in both the left and right hemispheres compared to the passive listening tasks. Males were characterized by a significant increase in mu-beta ERD in the left hemisphere as task demands were increased.

Differential recruitment of the anterior dorsal stream due to increased task demands is a well-established phenomenon (Pulvermüller, 2013; Szenkovits et al., 2012). When active discrimination is required, late mu-alpha and mu-beta ERD is often linked to covert rehearsal of the stimuli, which is thought to be necessary to maintain information in working memory for the purpose of making a decision (Baddeley, 2012; Jenson et al., 2014; LoCasto et al., 2004; Thornton et al., 2017). Indeed, both males and females are characterized by stimulus offset ERD. Increased ERD as task demands are increased suggests that females recruit additional cortical resources in both the left and right anterior dorsal stream regions in order to maintain stimuli in working memory when some active decision must be made regarding stimulus content. Males, however, only appear to recruit additional resources in the beta band of the left hemisphere. This could support evidence that males are more left lateralized in active speech processing tasks (Kansaku, Yamaura, \& Kitazawa, 2000). 


\section{Auditory Alpha}

Within groups, both males and females display differences within the left auditory component cluster as task demands are increased from passive listening to active discrimination tasks (Figure 4-5). Only males displayed differences in the alpha band in the right auditory cluster (Figure 4-6). Alpha ERD during stimulus presentation was stronger in females between conditions grouped by task demands, and these differences extended in to the beta band. Between conditions in males, alpha ERS after stimulus offset was greater in the active discrimination conditions relative to passive listening.

Greater alpha ERD in females during stimulus presentation when task demands are increased is likely representative of attention allocation toward sensory information being processed in the auditory cortical regions (Jenson et al., 2014). In this study, active discrimination conditions increased task demands by requiring participants to not only attend to stimuli, but evaluate the stimuli for content and make a decision. Females appear to allocate additional processing resources to the auditory cortical region, at least in the left hemisphere, to evaluate stimuli for meaningful content in order to complete the task. Beta ERD, sometimes associated with attention across the sensorimotor cortical region (Jenson et al., 2014; MacLean, Arnell, \& Cote, 2012; van Ede et al., 2011), is also found alongside alpha ERD in the female group.

Males did not display increased alpha ERD in response to increased task demands, instead responding with increased alpha ERS after stimulus offset. Alpha ERS in the auditory cortex has been found in both speech production and perception tasks (Jenson et al., 2015). This activity, at least in speech production, has been attributed to speech induced suppression, which reduces self-generated input to the sensory system. In perception, however, no overt production occurs. However, the timing of this ERS suggests that covert replay for working memory maintenance in the anterior dorsal stream may still reflexively inhibit the auditory region for a similar purpose. Interestingly, while females do show strong alpha ERS in the active discrimination conditions, no differences was found between task demand groups in the female participant group for late alpha ERS. Taken together, while both males and females are differentially responsive to increases in task demands in the right auditory cortex, these differences are only present within each group, not between groups.

\section{Differential Effects of Speaker Sex Revealed Within Each Group}

In the absence of task considerations, within group differences based on sex of the speaker were investigated by combining the passive and active conditions of each speaker type $(\mathrm{PF}+\mathrm{DF}$ vs $\mathrm{PM}+\mathrm{DM})$. Significant differences within groups were found in the left and right mu clusters for both males and females. Both males and females were characterized by greater mu-beta ERD before, during, and after stimulus presentation when listening to males, while overall patterns of mu rhythm activity were consistent with previous works which found very little alpha activity or slight alpha ERS before stimulus onset, paired with beta ERD. Late alpha differences were noted within the male 
group was found post stimulus offset, specifically with greater alpha ERD compared to female speaker conditions.

Time-frequency analysis of the mu rhythm revealed broadly consistent patterns of activity in both males and females, with great mu-beta ERD throughout the time-course of the stimulus when listening to a male speaker in the left and right hemispheres (Figures 4-7 and 4-8). In the absence of behavioral differences, these ERD differences between speaker sexes suggest that male speakers necessitate additional resource allocation for early attention and continued processing throughout the stimulus presentation while maintaining the same behavioral outcome.

Junger and colleagues (2013) found some within group differences when males or females processed speakers of different sexes. Interestingly, this study found the females processing female voices more strongly than male voices. Despite the contradiction to the findings from this study, it is important to note that Junger and colleagues used a different methodology (fMRI) and revealed differences in different regions of the cortex (prefrontal and auditory cortices). These results, then, broadly agree with Junger and colleagues (2013) and show that regions of the brain may respond to different speaker sexes in different ways, within a group of listeners.

Other studies, not specifically focused on speech processing, have shown that the sex of the stimulus is not linked to mu rhythm reactivity. During observation of painful situation, a stimulus known to elicit mu rhythm suppression, sex of the stimulus (hand characteristics) did not alter mu rhythm response in either males or females (Cheng et al., 2008). It may be that Cheng and colleagues did not reveal within group differences between sex of the stimulus conditions due to reduced biological relevance of the stimulus relative to speech, however more investigation is necessary to determine mu reactivity differences between visual hand movements and speech sounds. Biological or evolutionary relevance becomes relevant as specifically opposite sex effects are thought to be relevant to mate selection (Smith et al., 2018). This explanation, however, would

suggest that males would be more responsive to a female speaker, and vice versa, which was not found in this study. Instead, both males and females appeared to preferentially process male speakers. Given the possibility of the influence of mate selection on cortical processing of speech signal, without data regarding the sexual orientation of the participants, it is possible that these findings may change if groups could be subdivided further (Smith et al., 2018).

\section{General Discussion}

This study sought to examine the effects of task demands, listener sex, and speaker sex on sensorimotor and auditory cortical activity using ICA derived components of EEG data. The pattern of group (male and female) differences across the anterior and posterior dorsal streams present compelling reason to consider that the bulk of data investigating "normal" activity in the brain across a variety of tasks may not accurately represent male and female activity. Rather, this data may represent a weakened pattern 
for both males and females. Considering specifically the results from the anterior dorsal stream region using time-frequency analysis, including males and females in one group may serve to move overall patterns of activation toward zero, when actual patterns of activity are simply moving to activation or inhibition in each group. The spectral power differences in the right auditory cluster which differentiate males and females also indicates that regional processing capacity differs between groups in this measure. Again suggesting that grouping of males and females may be detrimental to further understanding normal cortical processing of external stimuli.

Broadly, the results from this study suggest that when listening to speech stimuli, females tend to be more responsive to changes to the task demands, while males appears to be more sensitive to changes to the speaker sex. As with many studies that consider sex of the participant, within group variability is high, making these differences difficult to elicit. While this study is positioned comfortably alongside others in terms of participants in each group, and even in contributing data sets (Bowers et al., 2013; Jenson et al., 2014; Oberman et al., 2005; Pineda et al., 2013; Saltuklaroglu et al., 2017; Thornton et al., 2017), it may be necessary to pursue a much larger investigation to establish appropriate normative data when examining cortical activity using ICA/ERSP analysis of EEG data.

Informing current theoretical models regarding constructivist (i.e. analysis-bysynthesis) or direct realist models explaining recruitment of anterior dorsal stream regions during speech perception tasks is challenging for this data. Pre-stimulus mu-beta ERD is present in some conditions, suggesting that predictive coding may be present, supporting analysis-by-synthesis, where previous experiences may be used to constrain or focus analysis on possible candidate options regarding incoming stimuli (Jenson et al., 2014; Poeppel \& Monahan, 2011; Skipper et al., 2017, 2007). However, this pattern is more reliably visualized when listening to male speakers compared to female speakers, in both groups. When listening to females, however, anterior dorsal activation does not strongly occur prior to stimulus presentation in either group. This supports direct-realist theories that any activity in the sensorimotor region may indicate a direct auditory-tomotor mapping, the conversion of auditory information on to the motor gestures which would be used to internal recreate it (Fowler et al., 2003; Thornton et al., 2017). Taken together, then, it seems that speaker sex may influence processing strategy employed. Perhaps frequency characteristics of the speaker has an outsized impact on cortical processing. Speculatively, when performing tasks that have different speakers across the testing paradigm, processing may shift toward the right hemisphere, where identification of speaker sex may occur (Lattner, Meyer, \& Friederici, 2005).

The final goal of the study was to provide insight in to clinical disorders which are linked by a higher prevalence in males and sensorimotor disorder/dysfunction. The results from this study did reveal, at least across the anterior dorsal stream, some patterns of increased ERS in females. However, the extent of the ERS was not sufficient to reach significance when compared to patterns of male ERD early in stimulus presentation (just prior to and during) in most conditions. Increased ERS was present in females during discrimination of a male speaker in previous works (Thornton et al., 2019). In this study, 
greater ERS was found in females relative to males only when passively listening to male speakers, and only in the right hemisphere mu component. Reduced sensorimotor inhibition has been linked to several disorders that are more prevalent in males (Daliri \& Max, 2015; Daliri, Wieland, Cai, Guenther, \& Chang, 2017; Mollaei, Shiller, \& Gracco, 2013; Saltuklaroglu et al., 2017; Vollebregt, Zumer, ter Huurne, Buitelaar, \& Jensen, 2016), as such, reduced inhibitory capacity in non-clinical males may suggest that measures of alpha inhibition across the sensorimotor region may be clinically significant. In addition to these considerations, it may be important to consider that the findings from this study may at least partially explain the high variability in findings across neuroimaging studies that feature males and females in the same population.

Sensorimotor regions of the dorsal stream are thought to be influenced by basal ganglia activity due to their anatomical connections (Alexander, DeLong, \& Strick, 1986). Many disorders that have a documented male bias are also linked to dysfunction in the basal ganglia (Alm, 2004; Blandini, Nappi, Tassorelli, \& Martignoni, 2000; Silk, Vance, Rinehart, Bradshaw, \& Cunnington, 2009). Given this, it is possible that sexrelated differences measured in the anterior dorsal stream may be reflective of sex-related differences in the basal ganglia, and may explain some of the symptoms and presentation of certain disorders such as stuttering.

Though no significant differences were revealed between males and females in this study, it is possible that investigation of activity in the basal ganglia could reveal such differences. EEG is not the ideal tool for investigation of these deeper brain structures, though several studies have suggested that surface recorded EEG signals can be localized to structures as deep as the hippocampus (Buzsáki, 2002; Vanneste \& De Ridder, 2016). Nonetheless, sex-related differences have been revealed in portions of the basal ganglia using other neuroimaging techniques, suggesting that neural circuits which recruit the basal ganglia also may be similarly sexually dimorphic in either structure or function (Rijpkema et al., 2012).

The basal ganglia are often strongly referenced by models of cognition, wherein they are linked to initiation and inhibition of action. As such, influence on cortical activity arises from these structures may be reflected as increases in inhibitory activity recorded across the anterior dorsal stream (Bonstrup, Hagemann, Gerloff, Sauseng, \& Hummel, 2015). This inhibitory activity could encode memory traces which are informed over repeated exposures to the same stimuli (Bowers et al., 2013; Jenson et al., 2014) or during training (Sauseng, Gerloff, \& Hummel, 2013). However, this study is unable to inform either of these possibilities, as limited ERS was found regardless of cortical region examined. It is possible that the paradigm employed by this study did not sufficiently tax these corticobasal-ganglia and thalamocortical circuits to elicit sex-related differences that could then be detected downstream at the level of the sensorimotor mu rhythm. Despite the evidence of anatomical differences (Rijpkema et al., 2012), additional investigation is necessary to establish functional basal ganglia differences related to sex. 


\section{Limitations}

The current study successful identified the effects of task, listener sex, and speaker sex effects on both the anterior and posterior dorsal stream regions, however, it is not without limitations. As with many studies utilizing these methods, not all participants contribute usable data, and not all participants contribute components to the mu and auditory clusters. The cluster contribution numbers are in line with a previous studies examining mu (Bowers et al., 2013; Thornton et al., 2017) and auditory activity in speech perception (Jenson et al., 2015), though they are rather low overall, particularly in the auditory cluster, and suggest the need for larger studies to better characterize sex-related differences in the brain.

It is also worth noting that emerging evidence suggests that sex and sexuality may influence cortical processing within regions of the cortex, or even which regions of the cortex are active during a task. This study focused on investigation of sex-related differences only, and did not control for sexuality. Given the results from a recent study from Smith and colleagues (2019), homosexual males and homosexual females may be characterized by differences in cortical activity than their same-sex heterosexual counterparts. The likelihood that the current study includes at least 2 homosexual males and 3 homosexual females is high, given the prevalence of homosexuality reported at roughly $\sim 10 \%$ for males and females in the United States (Sell, Wells, \& Wypij, 1995). Without such demographic information, actual participant inclusion of this population could be much higher. The effects on the data could decrease likelihood of significant differences, based on the results from Smith and colleagues (2019). 


\section{LIST OF REFERENCES}

Aerts, A., van Mierlo, P., Hartsuiker, R. J., Santens, P., \& De Letter, M. (2015). Sex Differences in Neurophysiological Activation Patterns During Phonological Input Processing: An Influencing Factor for Normative Data. Archives of Sexual Behavior, 44(8), 2207-2218. https://doi.org/10.1007/s10508-015-0560-y

Alexander, G. E., DeLong, M. R., \& Strick, P. L. (1986). Parallel organization of functionally segregated circuits linking basal ganglia and cortex. Annual Review of Neuroscience, 9, 357-381. https://doi.org/10.1146/annurev.ne.09.030186.002041

Alm, P. A. (2004). Stuttering and the basal ganglia circuits: a critical review of possible relations. Journal of Communication Disorders, 37(4), 325-369. https://doi.org/10.1016/j.jcomdis.2004.03.001

Arnal, L. H. (2012). Predicting "When” Using the Motor System's Beta-Band Oscillations. Frontiers in Human Neuroscience, 6(August), 1-3. https://doi.org/10.3389/fnhum.2012.00225

Arnal, L. H., Giraud, A.-L., Friston, K., Lochmann, T., Deneve, S., Knill, D. C., ... Miller, E. K. (2012). Cortical oscillations and sensory predictions. Trends in Cognitive Sciences, 16(7), 390-398. https://doi.org/10.1016/j.tics.2012.05.003

Baddeley, A. (2012). Working Memory: Theories, Models, and Controversies. Annual Review of Psychology, 63(1), 1-29. https://doi.org/10.1146/annurev-psych-120710100422

Bartoli, E., D’Ausilio, A., Berry, J., Badino, L., Bever, T., \& Fadiga, L. (2015). Listenerspeaker perceived distance predicts the degree of motor contribution to speech perception. Cerebral Cortex, 25(2), 281-288. https://doi.org/10.1093/cercor/bht257

Belin, P., Zatorre, R. J., \& Ahad, P. (2002). Human temporal-lobe response to vocal sounds. Brain Research. Cognitive Brain Research, 13(1), 17-26.

Belin, P., Zatorre, R. J., Lafaille, P., Ahad, P., \& Pike, B. (2000). Voice-selective areas in human auditory cortex. Nature, 403(6767), 309-312. https://doi.org/10.1038/35002078

Blandini, F., Nappi, G., Tassorelli, C., \& Martignoni, E. (2000). Functional changes of the basal ganglia circuitry in Parkinson's disease. Progress in Neurobiology, 62(1), 63-88.

Bonnefond, M., \& Jensen, O. (2012). Alpha oscillations serve to protect working memory maintenance against anticipated distracters. Current Biology, 22(20), 1969-1974. https://doi.org/10.1016/j.cub.2012.08.029 
Bonstrup, M., Hagemann, J., Gerloff, C., Sauseng, P., \& Hummel, F. C. (2015). Alpha oscillatory correlates of motor inhibition in the aged brain. Frontiers in Aging Neuroscience, 7(OCT), 1-10. https://doi.org/10.3389/fnagi.2015.00193

Bouton, S., Chambon, V., Tyrand, R., Guggisberg, A. G., Seeck, M., Karkar, S., ... Giraud, A.-L. (2018). Focal versus distributed temporal cortex activity for speech sound category assignment. Proceedings of the National Academy of Sciences of the United States of America, 1299-1308. https://doi.org/https://doi.org/10.1101/133272

Bowers, A., Saltuklaroglu, T., Harkrider, A., \& Cuellar, M. (2013). Suppression of the $\mu$ Rhythm during Speech and Non-Speech Discrimination Revealed by Independent Component Analysis: Implications for Sensorimotor Integration in Speech Processing. PLoS ONE, 8(8). https://doi.org/10.1371/journal.pone.0072024

Brinkman, L., Stolk, A., Dijkerman, H. C., de Lange, F. P., \& Toni, I. (2014). Distinct roles for alpha- and beta-band oscillations during mental simulation of goal-directed actions. J Neurosci, 34(44), 14783-14792.

https://doi.org/10.1523/JNEUROSCI.2039-14.2014

Burton, M. W. (2001). The role of inferior frontal cortex in phonological processing. Cognitive Science, 25(5), 695-709. https://doi.org/10.1016/S0364-0213(01)00051-9

Burton, M. W., \& Small, S. L. (2006). Functional neuroanatomy of segmenting speech and nonspeech. Cortex, 42(4), 644-651. https://doi.org/10.1016/S00109452(08)70400-3

Burton, M. W., Small, S. L., \& Blumstein, S. E. (2000). The role of segmentation in phonological processing: an fMRI investigation. Journal of Cognitive Neuroscience, 12(4), 679-690. https://doi.org/10.1162/089892900562309

Buzsáki, G. (2002). Theta oscillations in the hippocampus. Neuron, 33(3), 325-340. https://doi.org/10.1016/S0896-6273(02)00586-X

Buzsáki, G. (2009). Rhythms of the Brain. Rhythms of the Brain. https://doi.org/10.1093/acprof:oso/9780195301069.001.0001

Callan, D., Callan, A., Gamez, M., Sato, M. aki, \& Kawato, M. (2010). Premotor cortex mediates perceptual performance. NeuroImage, 51(2), 844-858. https://doi.org/10.1016/j.neuroimage.2010.02.027

Callan, D., Callan, A., \& Jones, J. A. (2014). Speech motor brain regions are differentially recruited during perception of native and foreign-accented phonemes for first and second language listeners. Frontiers in Neuroscience, 8(SEP), 1-15. https://doi.org/10.3389/fnins.2014.00275 
Cannon, E. N., Yoo, K. H., Vanderwert, R. E., Ferrari, P. F., Woodward, A. L., \& Fox, N. A. (2014). Action experience, more than observation, influences mu rhythm desynchronization. PLoS ONE, 9(3), 1-8.

https://doi.org/10.1371/journal.pone.0092002

Carlqvist, H., Nikulin, V. V., Str??mberg, J. O., \& Brismar, T. (2005). Amplitude and phase relationship between alpha and beta oscillations in the human electroencephalogram. Medical and Biological Engineering and Computing, 43(5), 599-607. https://doi.org/10.1007/BF02351033

Centeno, M., Koepp, M. J., Vollmar, C., Stretton, J., Sidhu, M., Michallef, C., ... Duncan, J. S. (2014). Language dominance assessment in a bilingual population: Validity of fMRI in the second language. Epilepsia, 55(10), 1504-1511. https://doi.org/10.1111/epi.12757

Chen, Y., \& Huang, X. (2016). Modulation of alpha and beta oscillations during an nback task with varying temporal memory load. Frontiers in Psychology, 6(JAN), 110. https://doi.org/10.3389/fpsyg.2015.02031

Cheng, Y., Chou, K. H., Decety, J., Chen, I. Y., Hung, D., Tzeng, O. J. L., \& Lin, C. P. (2009). Sex differences in the neuroanatomy of human mirror-neuron system: A voxel-based morphometric investigation. Neuroscience, 158(2), 713-720. https://doi.org/10.1016/j.neuroscience.2008.10.026

Cheng, Y., Lee, P. L., Yang, C. Y., Lin, C. P., Hung, D., \& Decety, J. (2008). Gender differences in the mu rhythm of the human mirror-neuron system. PLoS ONE, 3(5), 1-7. https://doi.org/10.1371/journal.pone.0002113

Cheng, Y., Tzeng, O. J. L., Decety, J., Imada, T., \& Hsieh, J.-C. (2006). Gender Differences in the human mirror system: a magnetoencephalographic study. Neuroreport, 7(11), 1115-1119.

Chevillet, M. A., Jiang, X., Rauschecker, J. P., \& Riesenhuber, M. (2013). Automatic phoneme category selectivity in the dorsal auditory stream. Annals of Internal Medicine, 158(6), 5208-5215. https://doi.org/10.1523/JNEUROSCI.1870-12.2013

Crawcour, S., Bowers, A., Harkrider, A., \& Saltuklaroglu, T. (2009). Mu wave suppression during the perception of meaningless syllables: EEG evidence of motor recruitment. Neuropsychologia, 47(12), 2558-2563. https://doi.org/10.1016/j.neuropsychologia.2009.05.001

Cuellar, M., Bowers, A., Harkrider, A. W., Wilson, M., \& Saltuklaroglu, T. (2012). Mu suppression as an index of sensorimotor contributions to speech processing: Evidence from continuous EEG signals. International Journal of Psychophysiology, 85(2), 242-248. https://doi.org/10.1016/j.ijpsycho.2012.04.003 
Cuellar, M. E., \& del Toro, C. M. (2017). Time-frequency analysis of Mu rhythm activity during picture and video action naming tasks. Brain Sciences, 7(9). https://doi.org/10.3390/brainsci7090114

Cuellar, M., Harkrider, A. W., Jenson, D., Thornton, D., Bowers, A., \& Saltuklaroglu, T. (2016). Time-frequency analysis of the EEG mu rhythm as a measure of sensorimotor integration in the later stages of swallowing. Clinical Neurophysiology, 127(7), 2625-2635. https://doi.org/10.1016/j.clinph.2016.04.027

D’Ausilio, A., Bufalari, I., Salmas, P., \& Fadiga, L. (2012). The role of the motor system in discriminating normal and degraded speech sounds. Cortex, 48(7), 882-887. https://doi.org/10.1016/j.cortex.2011.05.017

D’Ausilio, A., Pulverm??1ler, F., Salmas, P., Bufalari, I., Begliomini, C., \& Fadiga, L. (2009). The Motor Somatotopy of Speech Perception. Current Biology, 19(5), 381385. https://doi.org/10.1016/j.cub.2009.01.017

D’Ausilio, A., Pulvermüller, F., Salmas, P., Bufalari, I., Begliomini, C., \& Fadiga, L. (2009). The Motor Somatotopy of Speech Perception. Current Biology, 19(5), 381385. https://doi.org/10.1016/j.cub.2009.01.017

Daliri, A., \& Max, L. (2015). Electrophysiological evidence for a general auditory prediction deficit in adults who stutter. Brain and Language, 150, 37-44. https://doi.org/10.1016/j.bandl.2015.08.008

Daliri, A., Wieland, E. A., Cai, S., Guenther, F. H., \& Chang, S.-E. (2017). Auditorymotor adaptation is reduced in adults who stutter but not in children who stutter. Developmental Science, (January 2016), e12521. https://doi.org/10.1111/desc.12521

De Ridder, D., Congedo, M., \& Vanneste, S. (2015). The neural correlates of subjectively perceived and passively matched loudness perception in auditory phantom perception. Brain and Behavior, 5(5), 1-12. https://doi.org/10.1002/brb3.331

De Ridder, D., Vanneste, S., Langguth, B., \& Llinas, R. (2015). Thalamocortical dysrhythmia: A theoretical update in tinnitus. Frontiers in Neurology, 6(MAY), 113. https://doi.org/10.3389/fneur.2015.00124

Dehan, C. P., \& Jerger, J. (1990). Analysis of gender differences in the auditory brainstem response. The Laryngoscope, 100(1), 18-24. https://doi.org/10.1288/00005537-199001000-00005

Delorme, A., Palmer, J., Onton, J., Oostenveld, R., \& Makeig, S. (2012). Independent EEG sources are dipolar. PLoS ONE, 7(2). https://doi.org/10.1371/journal.pone.0030135 
Drew, A. R., Quandt, L. C., \& Marshall, P. J. (2015). Visual influences on sensorimotor EEG responses during observation of hand actions. Brain Research, 1597, 119-128. https://doi.org/10.1016/j.brainres.2014.11.048

Du, Y., Buchsbaum, B. R., Grady, C. L., \& Alain, C. (2014). Noise differentially impacts phoneme representations in the auditory and speech motor systems. Proceedings of the National Academy of Sciences, 111(19), 7126-7131. https://doi.org/10.1073/pnas.1318738111

Du, Y., Buchsbaum, B. R., Grady, C. L., \& Alain, C. (2016). Increased activity in frontal motor cortex compensates impaired speech perception in older adults. Nature Communications, 7(August), 12241. https://doi.org/10.1038/ncomms12241

Edlin, J. M., Leppanen, M. L., Fain, R. J., Hackländer, R. P., Hanaver-Torrez, S. D., \& Lyle, K. B. (2015). On the use (and misuse?) of the Edinburgh Handedness Inventory. Brain and Cognition, 94, 44-51. https://doi.org/10.1016/j.bandc.2015.01.003

Eriksson, J., Vogel, E. K., Lansner, A., Bergström, F., \& Nyberg, L. (2015). Neurocognitive Architecture of Working Memory. Neuron, 88(1), 33-46. https://doi.org/10.1016/j.neuron.2015.09.020

Fowler, C. A., Brown, J. M., Sabadini, L., \& Weihing, J. (2003). Rapid access to speech gestures in perception: Evidence from choice and simple response time tasks. Journal of Memory and Language, 49(3), 396-413. https://doi.org/10.1016/S0749596X(03)00072-X

Fox, N. A., Bakermans-Kranenburg, M. J., Yoo, K. H., Bowman, L. C., Cannon, E. N., Vanderwert, R. E., ... Van Ijzendoorn, M. H. (2016). Assessing Human Mirror Activity With EEG Mu Rhythm: A Meta-Analysis. Psychological Bulletin, 142(3), 291-313. https://doi.org/10.1037/bul0000031

Foxe, J. J., \& Snyder, A. C. (2011). The role of alpha-band brain oscillations as a sensory suppression mechanism during selective attention. Frontiers in Psychology, 2(JUL), 1-13. https://doi.org/10.3389/fpsyg.2011.00154

Frühholz, S., Gschwind, M., \& Grandjean, D. (2015). Bilateral dorsal and ventral fiber pathways for the processing of affective prosody identified by probabilistic fiber tracking. NeuroImage, 109, 27-34.

https://doi.org/10.1016/j.neuroimage.2015.01.016

Fujioka, T., Ross, B., \& Trainor, L. J. (2015). Beta-Band Oscillations Represent Auditory Beat and Its Metrical Hierarchy in Perception and Imagery. Journal of Neuroscience, 35(45), 15187-15198. https://doi.org/10.1523/JNEUROSCI.239715.2015 
Galantucci, B., Fowler, C., \& Turvey, M. T. (2006). The motor theory of speech perception reviewed. Psychonomic Bulletin \& Review, 13(3), 361-377. https://doi.org/10.1097/MPG.0b013e3181a15ae8.Screening

Georgiev, S., Minchev, Z., Christova, C., \& Philipova, D. (2011). Gender event-related brain oscillatory differences in normal elderly population EEG. International Journal Bioautomation, 15(1), 33-48.

Gola, M., Magnuski, M., Szumska, I., \& Wróbel, A. (2013). EEG beta band activity is related to attention and attentional deficits in the visual performance of elderly subjects. International Journal of Psychophysiology, 89(3), 334-341. https://doi.org/10.1016/j.ijpsycho.2013.05.007

Gootjes, L., Bouma, A., Van Strien, J. W., Scheltens, P., \& Stam, C. J. (2006). Attention modulates hemispheric differences in functional connectivity: Evidence from MEG recordings. NeuroImage, 30(1), 245-253.

https://doi.org/10.1016/j.neuroimage.2005.09.015

Graimann, B., \& Pfurtscheller, G. (2006). Quantification and visualization of eventrelated changes in oscillatory brain activity in the time-frequency domain. Progress in Brain Research, 159, 79-97. https://doi.org/10.1016/S0079-6123(06)59006-5

Gur, R. C., Alsop, D., Glahn, D., Petty, R., Swanson, C. L., Maldjian, J. A., ... Gur, R. E. (2000). An fMRI study of sex differences in regional activation to a verbal and a spatial task. Brain and Language, 74(2), 157-170.

https://doi.org/10.1006/brln.2000.2325

Hanslmayr, S., Gross, J., Klimesch, W., \& Shapiro, K. L. (2011). The role of alpha oscillations in temporal attention. Brain Research Reviews, 67(1-2), 331-343. https://doi.org/10.1016/j.brainresrev.2011.04.002

Hari, R. (2006). Action-perception connection and the cortical mu rhythm. Progress in Brain Research, 159, 253-260. https://doi.org/10.1016/S0079-6123(06)59017-X

Hickok, G., Costanzo, M., \& Capasso, R. (2011, December). The Role of Broca's area in Speech Perception: Evidence from Aphasia Revisited. https://doi.org/10.1016/j.bandl.2011.08.001

Hickok, G., \& Poeppel, D. (2000). Towards a functional neuroanatomy of speech perception. Trends in Cognitive Sciences, 4(4), 131-138. https://doi.org/10.1016/S1364-6613(00)01463-7

Hickok, G., \& Poeppel, D. (2004). Dorsal and ventral streams: A framework for understanding aspects of the functional anatomy of language. Cognition, 92(1-2), 67-99. https://doi.org/10.1016/j.cognition.2003.10.011 
Hickok, G., \& Poeppel, D. (2007). The cortical organization of speech processing. Nature Reviews. Neuroscience, 8(5), 393-402. https://doi.org/10.1038/nrn2113

Hillenbrand, J., Getty, L. A., Clark, M. J., \& Wheeler, K. (1995). Acoustic characteristics of American English vowels. The Journal of the Acoustical Society of America, 97(5 Pt 1), 3099-3111.

Hobson, H. M., \& Bishop, D. V. M. (2016). Mu suppression - A good measure of the human mirror neuron system? Cortex, 82, 290-310. https://doi.org/10.1016/j.cortex.2016.03.019

Hobson, H. M., \& Bishop, D. V. M. (2017). The interpretation of mu suppression as an index of mirror neuron activity: Past, present and future. Royal Society Open Science, 4(3). https://doi.org/10.1098/rsos.160662

Jain, A., Bansal, R., Kumar, A., \& Singh, K. D. (2015). A comparative study of visual and auditory reaction times on the basis of gender and physical activity levels of medical first year students. International Journal of Applied and Basic Medical Research. India. https://doi.org/10.4103/2229-516X.157168

Jensen, O., Gelfand, J., Kounios, J., \& Lisman, J. E. (2002). Oscillations in the alpha band $(9-12 \mathrm{~Hz})$ increase with memory load during retention in a short-term memory task. Cerebral Cortex, 12(8), 877-882. https://doi.org/10.1093/cercor/12.8.877

Jensen, O., \& Mazaheri, A. (2010). Shaping functional architecture by oscillatory alpha activity: gating by inhibition. Frontiers in Human Neuroscience, 4(November), 186. https://doi.org/10.3389/fnhum.2010.00186

Jenson, D., Bowers, A. L., Harkrider, A. W., Thornton, D., Cuellar, M., \& Saltuklaroglu, T. (2014). Temporal dynamics of sensorimotor integration in speech perception and production: Independent component analysis of EEG data. Frontiers in Psychology, 5(JUL), 1-17. https://doi.org/10.3389/fpsyg.2014.00656

Jenson, D., Harkrider, A. W., Thornton, D., Bowers, A. L., \& Saltuklaroglu, T. (2015a). Auditory cortical deactivation during speech production and following speech perception: an EEG investigation of the temporal dynamics of the auditory alpha rhythm. Frontiers in Human Neuroscience, 9(October), 534. https://doi.org/10.3389/fnhum.2015.00534

Jones, S. R., Pritchett, D. L., Sikora, M. a, Stufflebeam, S. M., Hämäläinen, M., \& Moore, C. I. (2009). Quantitative analysis and biophysically realistic neural modeling of the MEG mu rhythm: rhythmogenesis and modulation of sensoryevoked responses. Journal of Neurophysiology, 102(6), 3554-3572. https://doi.org/10.1152/jn.00535.2009 
Junger, J., Pauly, K., Bröhr, S., Birkholz, P., Neuschaefer-Rube, C., Kohler, C., ... Habel, U. (2013). Sex matters: Neural correlates of voice gender perception. NeuroImage, 79, 275-287. https://doi.org/10.1016/j.neuroimage.2013.04.105

Kandylaki, K. D., Nagels, A., Tune, S., Kircher, T., Wiese, R., Schlesewsky, M., \& Bornkessel-Schlesewsky, I. (2016). Predicting "When" in Discourse Engages the Human Dorsal Auditory Stream: An fMRI Study Using Naturalistic Stories. Journal of Neuroscience, 36(48), 12180-12191. https://doi.org/10.1523/JNEUROSCI.410015.2016

Kansaku, K., Yamaura, A., \& Kitazawa, S. (2000). Sex Differences in Lateralization Revealed in the Posterior Language Areas, 866-872.

Kevan, A., \& Pammer, K. (2009). Predicting early reading skills from pre-reading measures of dorsal stream functioning. Neuropsychologia, 47(14), 3174-3181. https://doi.org/10.1016/j.neuropsychologia.2009.07.016

Kittilstved, T., Reilly, K. J., Harkrider, A. W., Casenhiser, D., Thornton, D., Jenson, D. E., ... Saltuklaroglu, T. (2018). The Effects of Fluency Enhancing Conditions on Sensorimotor Control of Speech in Typically Fluent Speakers : An EEG Mu Rhythm Study, 12(April), 1-15. https://doi.org/10.3389/fnhum.2018.00126

Kleinsorge, T., \& Scheil, J. (2016). Guessing versus Choosing an Upcoming Task. Frontiers in Psychology, 7(March), 396. https://doi.org/10.3389/fpsyg.2016.00396

Klimesch, W. (2012). Alpha-band oscillations, attention, and controlled access to stored information. Trends in Cognitive Sciences, 16(12), 606-617. https://doi.org/10.1016/j.tics.2012.10.007

Knyazev, G. G. (2007). Motivation, emotion, and their inhibitory control mirrored in brain oscillations. Neuroscience and Biobehavioral Reviews, 31(3), 377-395. https://doi.org/10.1016/j.neubiorev.2006.10.004

Koshino, H., Minamoto, T., Yaoi, K., Osaka, M., \& Osaka, N. (2014). Coactivation of the default mode network regions and working memory network regions during task preparation. Scientific Reports, 4, 34-39. https://doi.org/10.1038/srep05954

Kotz, S. A., D’Ausilio, A., Raettig, T., Begliomini, C., Craighero, L., Fabbri-Destro, M., ... Fadiga, L. (2010). Lexicality drives audio-motor transformations in Broca's area. Brain and Language, 112(1), 3-11. https://doi.org/10.1016/j.bandl.2009.07.008

Kumar, S., Joseph, S., Gander, P. E., Barascud, N., Halpern, A. R., \& Griffiths, T. D. (2016). A Brain System for Auditory Working Memory. Journal of Neuroscience, 36(16), 4492-4505. https://doi.org/10.1523/JNEUROSCI.4341-14.2016 
Lattner, S., Meyer, M. E., \& Friederici, A. D. (2005). Voice perception: Sex, pitch, and the right hemisphere. Human Brain Mapping, 24(1), 11-20.

https://doi.org/10.1002/hbm.20065

Leaver, A. M., Seydell-Greenwald, A., \& Rauschecker, J. P. (2016). Auditory-limbic interactions in chronic tinnitus: Challenges for neuroimaging research. Hearing Research, 334, 49-57. https://doi.org/10.1016/j.heares.2015.08.005

Lehtelä, L., Salmelin, R., \& Hari, R. (1997). Evidence for reactive magnetic 10-Hz rhythm in the human auditory cortex. Neuroscience Letters, 222(2), 111-114. https://doi.org/10.1016/S0304-3940(97)13361-4

Li, R. (2014). Why women see differently from the way men see? A review of sex differences in cognition and sports. Journal of Sport and Health Science, 3(3), 155162. https://doi.org/10.1016/j.jshs.2014.03.012

Li, Y., Gu, F., Zhang, X., Yang, L., Chen, L., Wei, Z., ... Zhang, X. (2014). Cerebral activity to opposite-sex voices reflected by event-related potentials. PLOS ONE, 9(4). https://doi.org/10.1371/journal.pone.0094976

Liberman, A., \& Mattingly, I. (1985). The motor theory of speech perception revisited. Psychonomic Bulletin and Review, 15(2), 453-457. https://doi.org/10.3758/PBR.15.2.453

Liebenthal, E., Sabri, M., Beardsley, S. a, Mangalathu-Arumana, J., \& Desai, A. (2013). Neural dynamics of phonological processing in the dorsal auditory stream. The Journal of Neuroscience : The Official Journal of the Society for Neuroscience, 33(39), 15414-15424. https://doi.org/10.1523/JNEUROSCI.1511-13.2013

Lisker, L., \& Abramson, A. S. (1967). Some effects of context on voice onset time in English stops. Language and Speech, 10(1), 1-28. https://doi.org/10.1177/002383096701000101

LoCasto, P. C., Krebs-Noble, D., Gullapalli, R. P., \& Burton, M. W. (2004). An fMRI investigation of speech and tone segmentation. Journal of Cognitive Neuroscience, 16(9), 1612-1624. https://doi.org/10.1162/0898929042568433

Lundqvist, M., Rose, J., Herman, P., Brincat, S. L., Buschman, T. J., \& Miller, E. K. (2015). Gamma and Beta Bursts Underlie Working Memory. Neuron, 90(1), 152164. https://doi.org/10.1016/j.neuron.2016.02.028

MacLean, M. H., Arnell, K. M., \& Cote, K. A. (2012). Resting EEG in alpha and beta bands predicts individual differences in attentional blink magnitude. Brain and Cognition, 78(3), 218-229. https://doi.org/10.1016/j.bandc.2011.12.010 
Marshall, P. J., \& Meltzoff, A. N. (2012). Neural Mirroring Systems: Exploring the EEG $\mathrm{Mu}$ Rhythm in Human Infancy. Developmental Cognitive Neuroscience, 1(2), 110 123. https://doi.org/10.1016/j.den.2010.09.001.Neural

Mathewson, K. E., Beck, D. M., Ro, T., Maclin, E. L., Low, K. A., Fabiani1, M., \& Gratton, G. (2014). Dynamics of Alpha Control: Preparatory Suppression of Posterior Alpha Oscillations by Frontal Modulators Revealed with Combined EEG and Event-related Optical Signal. Journal of Cognitive Neuroscience, 26(10), 24002415. https://doi.org/10.1162/jocn

Matsumoto, J., Fujiwara, T., Takahashi, O., Liu, M., Kimura, A., \& Ushiba, J. (2010). Modulation of mu rhythm desynchronization during motor imagery by transcranial direct current stimulation. Journal of Neuroengineering and Rehabilitation, 7, 27. https://doi.org/10.1186/1743-0003-7-27

Mazaheri, A., van Schouwenburg, M. R., Dimitrijevic, A., Denys, D., Cools, R., \& Jensen, O. (2014). Region-specific modulations in oscillatory alpha activity serve to facilitate processing in the visual and auditory modalities. NeuroImage, 87, 356362. https://doi.org/10.1016/j.neuroimage.2013.10.052

McFadden, D. (1998). Sex differences in the auditory system. Developmental Neuropsychology, 14(2-3), 261-298. https://doi.org/10.1080/87565649809540712

Meister, I. G., Buelte, D., Staedtgen, M., Boroojerdi, B., \& Sparing, R. (2009). The dorsal premotor cortex orchestrates concurrent speech and fingertapping movements. European Journal of Neuroscience, 29(10), 2074-2082. https://doi.org/10.1111/j.1460-9568.2009.06729.x

Meyer, M., Luethi, M. S., Neff, P., Langer, N., \& Bochi, S. (2014). Disentangling tinnitus distress and tinnitus presence by means of EEG power analysis. Neural Plasticity, 2014. https://doi.org/10.1155/2014/468546

Minichino, A., Singh, F., Pineda, J., Friederich, E., \& Cadenhead, K. S. (2016). Biological Motion induced mu suppression is reduced in Early Psychosis (EP) patients with active negative symptoms and Autism Spectrum Disorders (ASD). Psychiatry Research, 238, 374-377. https://doi.org/10.1016/j.psychres.2016.01.057

Mollaei, F., Shiller, D. M., \& Gracco, V. L. (2013). Sensorimotor adaptation of speech in Parkinson's disease. Movement Disorders, 28(12), 1668-1674. https://doi.org/10.1002/mds.25588

Moore, A., Gorodnitsky, I., \& Pineda, J. (2012). EEG mu component responses to viewing emotional faces. Behavioural Brain Research, 226(1), 309-316. https://doi.org/10.1016/j.bbr.2011.07.048 
Möttönen, R., Dutton, R., \& Watkins, K. E. (2013). Auditory-motor processing of speech sounds. Cerebral Cortex, 23(5), 1190-1197. https://doi.org/10.1093/cercor/bhs110

Möttönen, R., Järveläinen, J., Sams, M., \& Hari, R. (2005). Viewing speech modulates activity in the left SI mouth cortex. NeuroImage, 24(3), 731-737. https://doi.org/10.1016/j.neuroimage.2004.10.011

Müller, N., \& Weisz, N. (2012). Lateralized auditory cortical alpha band activity and interregional connectivity pattern reflect anticipation of target sounds. Cerebral Cortex, 22(7), 1604-1613. https://doi.org/10.1093/cercor/bhr232

Murakami, T., Kell, C. A., Restle, J., Ugawa, Y., \& Ziemann, U. (2015). Left Dorsal Speech Stream Components and Their Contribution to Phonological Processing. Journal of Neuroscience, 35(4), 1411-1422. https://doi.org/10.1523/JNEUROSCI.0246-14.2015

Murphy, J. W., Foxe, J. J., Peters, J. B., \& Molholm, S. (2014). Susceptibility to distraction in autism spectrum disorder: Probing the integrity of oscillatory AlphaBand Suppression Mechanisms. Autism Research, 7(4), 442-458. https://doi.org/10.1002/aur.1374

Muthukumaraswamy, S. D., Johnson, B. W., Gaetz, W. C., \& Cheyne, D. O. (2004). Modulation of neuromagnetic oscillatory activity during the observation of orofacial movements. Neurology \& Clinical Neurophysiology : NCN, 2004, 2. https://doi.org/2 [pii]

Niedermeyer, E. (1990). Alpha-like rhythmical activity of the temporal lobe. Clinical EEG (Electroencephalography), 21(4), 210-224.

Nygaard, L. C., \& Pisoni, D. B. (1995). Chapter 3 - Speech Perception: New Directions in Research and Theory BT - Speech, Language, and Communication (2nd). In Handbook of Perception and Cognition (pp. 63-96). San Diego: Academic Press. https://doi.org/https://doi.org/10.1016/B978-012497770-9.50005-4

Nyström, P. (2008). The infant mirror neuron system studied with high density EEG. Social Neuroscience, 3(3-4), 334-347. https://doi.org/10.1080/17470910701563665

Oberman, L. M., Hubbard, E. M., McCleery, J. P., Altschuler, E. L., Ramachandran, V. S., \& Pineda, J. A. (2005). EEG evidence for mirror neuron dysfunction in autism spectrum disorders. Cognitive Brain Research, 24(2), 190-198. https://doi.org/10.1016/j.cogbrainres.2005.01.014

Obleser, J., \& Weisz, N. (2012). Suppressed alpha oscillations predict intelligibility of speech and its acoustic details. Cerebral Cortex, 22(11), 2466-2477. https://doi.org/10.1093/cercor/bhr325 
Ojanen, V., Möttönen, R., Pekkola, J., Jääskeläinen, I. P., Joensuu, R., Autti, T., \& Sams, M. (2005). Processing of audiovisual speech in Broca's area. NeuroImage, 25(2), 333-338. https://doi.org/10.1016/j.neuroimage.2004.12.001

Oldfield, R. C. (1971). the Assessment and Analysis of Handedness: the Edinburgh Inventory. Neuropsychologia, 9, 97-113.

Oostenveld, R., \& Oostendorp, T. F. (2002). Validating the boundary element method for forward and inverse EEG computations in the presence of a hole in the skull. Human Brain Mapping, 17(3), 179-192. https://doi.org/10.1002/hbm.10061

Osnes, B., Hugdahl, K., \& Specht, K. (2011). Effective connectivity analysis demonstrates involvement of premotor cortex during speech perception. NeuroImage, 54(3), 2437-2445. https://doi.org/10.1016/j.neuroimage.2010.09.078

Pani, P., Di Bello, F., Brunamonti, E., D’Andrea, V., Papazachariadis, O., \& Ferraina, S. (2014). Alpha- and beta-band oscillations subserve different processes in reactive control of limb movements. Frontiers in Behavioral Neuroscience, 8(November), 383. https://doi.org/10.3389/fnbeh.2014.00383

Pekkola, J., Laasonen, M., Ojanen, V., Autti, T., Jääskeläinen, I. P., Kujala, T., \& Sams, M. (2006). Perception of matching and conflicting audiovisual speech in dyslexic and fluent readers: An fMRI study at 3 T. NeuroImage, 29(3), 797-807. https://doi.org/10.1016/j.neuroimage.2005.09.069

Pernet, C. R., Latinus, M., Nichols, T. E., \& Rousselet, G. A. (2015, July). Cluster-based computational methods for mass univariate analyses of event-related brain potentials/fields: A simulation study. Journal of Neuroscience Methods. https://doi.org/10.1016/j.jneumeth.2014.08.003

Pessoa, L., Gutierrez, E., Bandettini, P., \& Ungerleider, L. (2002). Neural correlates of visual working memory: fMRI amplitude predicts task performance. Neuron, 35(5), 975-987. https://doi.org/10.1016/S0896-6273(02)00817-6

Peterson, S. E., Fox, P. T., Posner, M. I., Mintum, M., \& Raichle, M. E. (1988). Positron emission tomographic studies of the cortical anatomy of single-word processing. Nature, 331(6157), 585-589.

Pfurtscheller, G., \& Lopes, F. H. (1999). Event-related EEG / MEG synchronization and desynchronization : basic principles, 110, 1842-1857.

Pfurtscheller, G., Neuper, C., Andrew, C., \& Edlinger, G. (1997). Foot and hand area mu rhythms. International Journal of Psychophysiology, 26(1-3), 121-135.

https://doi.org/10.1016/S0167-8760(97)00760-5 
Pineda, J. a, Grichanik, M., Williams, V., Trieu, M., Chang, H., \& Keysers, C. (2013). EEG sensorimotor correlates of translating sounds into actions . Front Neurosci. , 7(1662-4548 (Print)), 203. https://doi.org/10.3389/fnins.2013.00203

Poeppel, D., \& Monahan, P. J. (2011). Feedforward and feedback in speech perception: Revisiting analysis by synthesis. Language and Cognitive Processes, 26(7), 935951. https://doi.org/10.1080/01690965.2010.493301

Popovich, C., Dockstader, C., Cheyne, D., \& Tannock, R. (2010). Sex differences in sensorimotor mu rhythms during selective attentional processing. Neuropsychologia, 48(14), 4102-4110. https://doi.org/10.1016/j.neuropsychologia.2010.10.016

Proverbio, A. M., Riva, F., Martin, E., \& Zani, A. (2010). Neural markers of opposite-sex bias in face processing. Frontiers in Psychology, 1(OCT), 1-12.

https://doi.org/10.3389/fpsyg.2010.00169

Pulvermüller, F. (2013). How neurons make meaning: Brain mechanisms for embodied and abstract-symbolic semantics. Trends in Cognitive Sciences, 17(9), 458-470. https://doi.org/10.1016/j.tics.2013.06.004

Pulvermuller, F., Huss, M., Kherif, F., Moscoso del Prado Martin, F., Hauk, O., \& Shtyrov, Y. (2006). Motor cortex maps articulatory features of speech sounds. Proceedings of the National Academy of Sciences, 103(20), 7865-7870. https://doi.org/10.1073/pnas.0509989103

Pulvermüller, F., Huss, M., Kherif, F., Moscoso del Prado Martin, F., Hauk, O., \& Shtyrov, Y. (2006). Motor cortex maps articulatory features of speech sounds. Proceedings of the National Academy of Sciences of the United States of America, 103(20), 7865-7870. https://doi.org/10.1073/pnas.0509989103

Quandt, L. C., \& Marshall, P. J. (2014). The effect of action experience on sensorimotor EEG rhythms during action observation. Neuropsychologia, 56(1), 401-408. https://doi.org/10.1016/j.neuropsychologia.2014.02.015

Quandt, L. C., Marshall, P. J., Bouquet, C. A., \& Shipley, T. F. (2013). Somatosensory experiences with action modulate alpha and beta power during subsequent action observation. Brain Research, 1534, 55-65. https://doi.org/10.1016/j.brainres.2013.08.043

Quandt, L. C., Marshall, P. J., Shipley, T. F., Beilock, S. L., \& Goldin-Meadow, S. (2012). Sensitivity of alpha and beta oscillations to sensorimotor characteristics of action: An EEG study of action production and gesture observation.

Neuropsychologia, 50(12), 2745-2751.

https://doi.org/10.1016/j.neuropsychologia.2012.08.005 
Rauschecker, J. P., \& Scott, S. K. (2009). Maps and streams in the auditory cortex: Nonhuman primates illuminate human speech processing. Nature Neuroscience, 12(6), 718-724. https://doi.org/10.1038/nn.2331

Rijpkema, M., Everaerd, D., van der Pol, C., Franke, B., Tendolkar, I., \& Fernández, G. (2012). Normal sexual dimorphism in the human basal ganglia. Human Brain Mapping, 33(5), 1246-1252. https://doi.org/10.1002/hbm.21283

Rogers, J. C., Mottonen, R., Boyles, R., \& Watkins, K. E. (2014). Discrimination of speech and non-speech sounds following theta-burst stimulation of the motor cortex. Frontiers in Psychology, 5(JUL), 1-13. https://doi.org/10.3389/fpsyg.2014.00754

Rohenkohl, G., \& Nobre, A. C. (2011). Alpha Oscillations Related to Anticipatory Attention Follow Temporal Expectations. Journal of Neuroscience, 31(40), 1407614084. https://doi.org/10.1523/JNEUROSCI.3387-11.2011

Ruigrok, A. N. V, Salimi-Khorshidi, G., Lai, M.-C., Baron-Cohen, S., Lombardo, M. V, Tait, R. J., \& Suckling, J. (2013). A meta-analysis of sex differences in human brain structure. Neuroscience and Biobehavioral Reviews, 39, 34-50. https://doi.org/10.1016/j.neubiorev.2013.12.004

Rüther, N. N., Brown, E. C., Klepp, A., \& Bellebaum, C. (2014). Observed manipulation of novel tools leads to mu rhythm suppression over sensory-motor cortices.

Behavioural Brain Research, 261, 328-335. https://doi.org/10.1016/j.bbr.2013.12.033

Ruytjens, L., Georgiadis, J. R., Holstege, G., Wit, H. P., Albers, F. W. J., \& Willemsen, A. T. M. (2007). Functional sex differences in human primary auditory cortex. European Journal of Nuclear Medicine and Molecular Imaging, 34(12), 2073-2081. https://doi.org/10.1007/s00259-007-0517-z

Sacchet, M. D., LaPlante, R. a, Wan, Q., Pritchett, D. L., Lee, A. K. C., Hämäläinen, M., ... Jones, S. R. (2015). Attention drives synchronization of alpha and beta rhythms between right inferior frontal and primary sensory neocortex. Journal of Neuroscience, 35(5), 2074-2082. https://doi.org/10.1523/JNEUROSCI.129214.2015

Salari, N., \& Rose, M. (2013). A brain-computer-interface for the detection and modulation of gamma band activity. Brain Sciences, 3(4), 1569-1587. https://doi.org/10.3390/brainsci3041569

Saltuklaroglu, T., Harkrider, A. W., Thornton, D., Jenson, D., \& Kittilstved, T. (2017). EEG $\mathrm{Mu}(\mu)$ rhythm spectra and oscillatory activity differentiate stuttering from non-stuttering adults. NeuroImage, (August 2016). https://doi.org/10.1016/j.neuroimage.2017.04.022 
Sauseng, P., Gerloff, C., \& Hummel, F. C. (2013). Two brakes are better than one: The neural bases of inhibitory control of motor memory traces. NeuroImage, 65, 52-58. https://doi.org/10.1016/j.neuroimage.2012.09.048

Savini, N., Brunetti, M., Babiloni, C., \& Ferretti, A. (2012). Working memory of somatosensory stimuli: An fMRI study. International Journal of Psychophysiology, 86(3), 220-228. https://doi.org/10.1016/j.ijpsycho.2012.09.007

Schnitzler, A., Gross, J., \& Timmermann, L. (2000). Synchronised oscillations of the human sensorimotor cortex. Acta Neurobiologiae Experimentalis, 60(2), 271-287. https://doi.org/10909184

Scott, S. K., McGettigan, C., \& Eisner, F. (2009). A little more conversation, a little less action - candidate roles for the motor cortex in speech perception. Nature Reviews Neuroscience, 10(4), 295-302. https://doi.org/10.1038/nrn2603

Seeber, M., Scherer, R., Wagner, J., Solis-Escalante, T., \& Muller-Putz, G. R. (2014). EEG beta suppression and low gamma modulation are different elements of human upright walking. Frontiers in Human Neuroscience, 8(July), 1-9. https://doi.org/10.3389/fnhum.2014.00485

Sell, R. L., Wells, J. A., \& Wypij, D. (1995). The prevalence of homosexual behavior and attraction in the United States, the United Kingdom and France: results of national population-based samples. Archives of Sexual Behavior, 24(3), 235-248.

Shahin, A. J., \& Pitt, M. A. (2012). Alpha activity marking word boundaries mediates speech segmentation. European Journal of Neuroscience, 36(12), 3740-3748. https://doi.org/10.1111/ejn.12008

Shaywitz, B. a, Shaywitz, S. E., Pugh, K. R., Constable, R. T., Skudlarski, P., Fulbright, R. K., ... Katz, L. (1995). Sex differences in the functional organization of the brain for language. Nature. https://doi.org/10.1038/373607a0

Silk, T. J., Vance, A., Rinehart, N., Bradshaw, J. L., \& Cunnington, R. (2009). Structural development of the basal ganglia in attention deficit hyperactivity disorder: a diffusion tensor imaging study. Psychiatry Research, 172(3), 220-225. https://doi.org/10.1016/j.pscychresns.2008.07.003

Skipper, J. I., Devlin, J. T., \& Lametti, D. R. (2017). The hearing ear is always found close to the speaking tongue: Review of the role of the motor system in speech perception. Brain and Language, 164, 77-105.

https://doi.org/10.1016/j.bandl.2016.10.004 
Skipper, J. I., Nusbaum, H. C., \& Small, S. L. (2005). Listening to talking faces: Motor cortical activation during speech perception. NeuroImage, 25(1), 76-89. https://doi.org/10.1016/j.neuroimage.2004.11.006

Skipper, J. I., Van Wassenhove, V., Nusbaum, H. C., \& Small, S. L. (2007). Hearing lips and seeing voices: How cortical areas supporting speech production mediate audiovisual speech perception. Cerebral Cortex, 17(10), 2387-2399. https://doi.org/10.1093/cercor/bhl147

Smith, E., Junger, J., Pauly, K., Kellermann, T., Dernt, B., \& Habel, U. (2018). Cerebral and behavioural response to human voices is mediated by sex and sexual orientation. Behavioural Brain Research, 356(July 2018), 89-97. https://doi.org/10.1016/j.bbr.2018.07.029

Szenkovits, G., Peelle, J. E., Norris, D., \& Davis, M. H. (2012). Individual differences in premotor and motor recruitment during speech perception. Neuropsychologia, 50(7), 1380-1392. https://doi.org/10.1016/j.neuropsychologia.2012.02.023

Thornton, D., Harkrider, A. W., Jenson, D. E., \& Saltuklaroglu, T. (2019). Sex differences in early sensorimotor processing for speech discrimination. Scientific Reports, 9(1), 392. https://doi.org/10.1038/s41598-018-36775-5

Thornton, D., Harkrider, A. W., Jenson, D., \& Saltuklaroglu, T. (2017). Sensorimotor activity measured via oscillations of EEG mu rhythms in speech and non-speech discrimination tasks with and without segmentation demands. Brain and Language. https://doi.org/10.1016/j.bandl.2017.03.011

Tiihonen, J., Hari, R., Kajola, M., Karhu, J., Ahlfors, S., \& Tissari, S. (1991). Magnetoencephalographic $10-\mathrm{Hz}$ rhythm from the human auditory cortex. Neuroscience Letters, 129(2), 303-305. https://doi.org/10.1016/03043940(91)90486-D

Trébuchon, A., Démonet, J. F., Chauvel, P., \& Liégeois-Chauvel, C. (2013). Ventral and dorsal pathways of speech perception: An intracerebral ERP study. Brain and Language, 127(2), 273-283. https://doi.org/10.1016/j.bandl.2013.04.007

Tzagarakis, C., Ince, N. F., Leuthold, A. C., \& Pellizzer, G. (2010). Beta-Band Activity during Motor Planning Reflects Response Uncertainty. Journal of Neuroscience, 30(34), 11270-11277. https://doi.org/10.1523/JNEUROSCI.6026-09.2010

Upadhayay, N. (2014). Comparison of Cognitive Functions Between Male and Female Medical Students: A Pilot Study. Journal of Clinical and Diagnostic Research, 8(6), 10-13. https://doi.org/10.7860/JCDR/2014/7490.4449 
van Ede, F., de Lange, F., Jensen, O., \& Maris, E. (2011). Orienting Attention to an Upcoming Tactile Event Involves a Spatially and Temporally Specific Modulation of Sensorimotor Alpha- and Beta-Band Oscillations. Journal of Neuroscience, 31(6), 2016-2024. https://doi.org/10.1523/JNEUROSCI.5630-10.2011

Vanneste, S., \& De Ridder, D. (2016). Deafferentation-based pathophysiological differences in phantom sound: Tinnitus with and without hearing loss. NeuroImage, 129, 80-94. https://doi.org/10.1016/j.neuroimage.2015.12.002

Vanneste, S., Song, J. J., \& De Ridder, D. (2013). Tinnitus and musical hallucinosis: The same but more. NeuroImage, 82, 373-383. https://doi.org/10.1016/j.neuroimage.2013.05.107

Vanneste, S., Van DeHeyning, P., \& DeRidder, D. (2015). Tinnitus: A large VBM-EEG correlational study. PLOS ONE, 10(3), 1-26. https://doi.org/10.1371/journal.pone.0115122

Venezia, J. H., Saberi, K., Chubb, C., \& Hickok, G. (2012). Response bias modulates the speech motor system during syllable discrimination. Frontiers in Psychology, 3(MAY), 1-13. https://doi.org/10.3389/fpsyg.2012.00157

Vollebregt, M. A., Zumer, J. M., ter Huurne, N., Buitelaar, J. K., \& Jensen, O. (2016). Posterior alpha oscillations reflect attentional problems in boys with Attention Deficit Hyperactivity Disorder. Clinical Neurophysiology, 127(5), 2182-2191. https://doi.org/10.1016/j.clinph.2016.01.021

Vossel, S., Geng, J. J., \& Fink, G. R. (2014). Dorsal and Ventral Attention Systems Distinct Neural Circuits but Collaborative Roles. The Neuroscientist, 20, 150-159. https://doi.org/10.1177/1073858413494269

Weisz, N., Hartmann, T., Müller, N., Lorenz, I., \& Obleser, J. (2011). Alpha rhythms in audition: Cognitive and clinical perspectives. Frontiers in Psychology, 2(APR), 115. https://doi.org/10.3389/fpsyg.2011.00073

Weisz, N., \& Obleser, J. (2014). Synchronisation signatures in the listening brain: A perspective from non-invasive neuroelectrophysiology. Hearing Research, 307, 1628. https://doi.org/10.1016/j.heares.2013.07.009

Wilson, S. M., \& Iacoboni, M. (2006). Neural responses to non-native phonemes varying in producibility: Evidence for the sensorimotor nature of speech perception. NeuroImage, 33(1), 316-325. https://doi.org/10.1016/j.neuroimage.2006.05.032

Wilson, S. M., Saygin, A. P., Sereno, M. I., \& Iacoboni, M. (2004). Listening to speech activates motor areas involved in speech production. Nature Neuroscience, 7(7), 701-702. https://doi.org/10.1038/nn1263 
Wu, C. C., Hamm, J. P., Lim, V. K., \& Kirk, I. J. (2016). Mu rhythm suppression demonstrates action representation in pianists during passive listening of piano melodies. Experimental Brain Research, 234(8), 2133-2139.

https://doi.org/10.1007/s00221-016-4615-7

Yang, C. Y., Decety, J., Lee, S., Chen, C., \& Cheng, Y. (2009). Gender differences in the mu rhythm during empathy for pain: An electroencephalographic study. Brain Research, 1251, 176-184. https://doi.org/10.1016/j.brainres.2008.11.062

Zumer, J. M., Scheeringa, R., Schoffelen, J. M., Norris, D. G., \& Jensen, O. (2014). Occipital Alpha Activity during Stimulus Processing Gates the Information Flow to Object-Selective Cortex. PLoS Biology, 12(10).

https://doi.org/10.1371/journal.pbio.1001965 


\section{VITA}

David M. Thornton was born in Seaside, California in 1990. He attended Longwood University in Farmville, Virginia, where he earned a Bachelor's Degree in Communication Sciences and Disorders in 2012. David then went to the University Tennessee Health Science Center to pursue a clinical doctoral degree in Audiology, earning a Master's Degree in Audiology in 2014. During the 2013 academic year, David worked under the direction of Dr. Ashley Harkrider in the Department of Audiology and Speech Pathology at the University Tennessee Health Science Center. David graduated in 2019 with a Doctor of Philosophy Degree in Speech and Hearing Science with a concentration in Hearing Science. 